\title{
Uso de modelos com fração de cura na análise de dados de sobrevivência com omissão nas covariáveis
}

\author{
Ângela Tavares Paes
}

TESE APRESENTADA

$\mathrm{AO}$

INSTITUTO DE MATEMÁTICA E ESTATÍSTICA

DA

UNIVERSIDADE DE SÃO PAULO

PARA

OBTENÇÃO DO TÍTULO DE DOUTOR

EM

CIENCIAS

\author{
Área de concentração: Estatística \\ Orientador: Prof. Dr. Antonio Carlos Pedroso de Lima
}

São Paulo, junho de 2007. 
Uso de modelos com fração de cura na análise de dados de sobrevivência com omissão nas covariáveis

Este exemplar corresponde à redação final da tese devidamente corrigida, defendida por Ângela Tavares Paes e aprovada pela comissão julgadora.

São Paulo, junho de 2007.

Banca examinadora:

- Prof. Dr. Antonio Carlos Pedroso de Lima

- Prof. Dr. Julio da Motta Singer

- Prof. Dr. Enrico Antonio Colosimo

- Prof. Dr. Antônio Eduardo Gomes

- Prof. Dra. Cicilia Yuko Wada 
Este foi um caminho de muitas escolhas e muitas mudanças de trajeto.

Ao terminar, a sensação que fica é que tudo está apenas começando.

Dedico esta tese ao Antonio Carlos. 


\section{Agradecimentos}

Ao Antonio Carlos, orientador e amigo, agradeço muitíssimo sua ajuda e paciência. Suas atitudes, postura profissional e sensibilidade foram essenciais para que eu concluísse este trabalho.

Ao Julio Singer, que eu sempre admirei e que sempre me orientou, desde a graduação até aqui. Foi muito bom tê-lo por perto com suas palavras firmes, principalmente nos momentos difíceis.

Ao Professor Clóvis A. Peres, pelo carinho, amizade e por ter me dado todo o apoio, permitindo que eu me ausentasse da UNIFESP.

Ao Humberto Freitas e Alfredo Mansur do Instituto do Coração, por terem gentilmente fornecido os dados que motivaram este trabalho.

À Professora Lúcia Barroso pela amizade e por ter participado da banca de qualificação.

Eu não teria concluído a parte de resultados sem a ajuda dos meus amigos e suas poderosas máquinas: Paulo Paiva, Liliam Lima, Rejane, Valdir, Kelsy e, em especial, ao David Pires, cuja contribuição foi fundamental para a conclusão das simulações.

Aos meus amigos do doutorado que acompanharam meus passos nessa jornada e com quem troquei muitas figurinhas, Mariana, Gisela, Iracema, Maria Paula, Adriana, Gleice, Edijane, Carine, Lilian Natis e Fred.

Aos meus pais, que mesmo sem entender muito bem o que é Estatística, sentem orgulho de mim e da minha profissão. À minha prima-irmã Maria e às meninas Natália, Nívea, Marcela e Marina. Sem o amor e os valores da minha família eu não chegaria à lugar nenhum.

Aos meus queridos amigos e confidentes Valdir, Helen, Cintia, Rê e Kelsy que cuidaram muito de mim e não deixaram que eu me privasse totalmente dos bons momentos da vida com boa música, boa comida, bons vinhos e boa conversa.

Ao meu companheiro Júnior e ao meu enteado Pedro. Aprendi muito com a nossa convivência e tive lições de vida muito mais importantes que qualquer lição dada em sala de aula.

Muito obrigada a todos ! 


\section{Resumo}

Em estudos cujo interesse é avaliar o efeito de fatores prognósticos sobre a sobrevida ou algum outro evento de interesse, é comum o uso de modelos de regressão que relacionam tempos de sobrevivência e covariáveis. Quando covariáveis que apresentam dados omissos são incluídas nos modelos de regressão, os programas estatísticos usuais automaticamente excluem aqueles indivíduos que apresentam omissão em pelo menos uma das covariáveis. Com isso, muitos pesquisadores utilizam apenas as observações completas, descartando grande parte da informação disponível. Está comprovado que a análise baseada apenas nos dados completos pode levar a estimadores altamente viesados e ineficientes. Para lidar com este problema, alguns métodos foram propostos na literatura. O objetivo deste trabalho é estender métodos que lidam com dados de sobrevivência e omissão nas covariáveis para a situação em que existe uma proporção de pacientes na população que não são suscetíveis ao evento de interesse. A idéia principal é utilizar modelos com fração de cura incluindo ponderações para compensar possíveis desproporcionalidades na subamostra de casos completos, levando-se em conta uma possível relação entre omissão e pior prognóstico. Foi considerado um modelo de mistura no qual os tempos de falha foram modelados através da família Weibull ou do modelo semiparamétrico de Cox e as probabilidade de cura foram especificadas por um modelo logístico. Os métodos propostos foram aplicados a dados reais, em que a omissão foi simulada em 10\%, 30\% e 50\% das observações. 


\begin{abstract}
Survival regression models are considered to evaluate the effect of prognostic factors for survival or some other event of interest. The standard statistical packages automatically exclude cases with at least one missing covariate value. Thus, many researchers use only the complete cases, discarding substantial part of the available information. It is known that this complete case analysis provides biased and inefficient estimates. The aim of this work is to extend survival models with missing covariate values to situations where some individuals are not susceptible to the event of interest. The main idea is to use cure rate models introducing individual weights to incorporate possible bias in the sample with complete cases, taking a possible relation between missingness and worse prognosis into account. Mixture models in which Weibull and Cox models are used to represent the failure times and logistic models to model the cure probabilities are considered. The performance of the procedure was evaluated via a simulation study. The proposed methods were applied to real data where the missingness was simulated in $10 \%, 30 \%$ and $50 \%$ of the observations.
\end{abstract}




\section{Índice}

1 Introdução 1

1.1 O problema de dados omissos . . . . . . . . . . . . . . . . . 2

1.2 Motivação . . . . . . . . . . . . . . . . . . . . . . . 2

1.3 Mecanismos de omissão . . . . . . . . . . . . . . . . . . . . . . 6

1.4 Revisão Bibliográfica . . . . . . . . . . . . . . . . . . . . . . . . . . 8

1.5 Proposta do trabalho . . . . . . . . . . . . . . . . . . 14

2 Modelos com fração de cura $\quad 16$

2.1 Formulação geral . . . . . . . . . . . . . . . . . . . . . . . 17

2.2 Família Weibull com fração de cura . . . . . . . . . . . . . . . . . 19

2.2.1 Estimação dos parâmetros . . . . . . . . . . . . . . . 20

2.3 Modelo semiparamétrico com fração de cura . . . . . . . . . . . . . . . 23

2.3.1 Estimação dos parâmetros - Algoritmo EM . . . . . . . . . . . . 27

3 Método da ponderação em modelos com fração de cura 38

3.1 Modelos ponderados . . . . . . . . . . . . . . . . . . . 39

3.1.1 Ponderação com base nas probabilidades de omissão . . . . . . . . . 40

3.1.2 Ponderação com base nas probabilidades de cura . . . . . . . . . . . 43

3.2 Modelos com fração de cura ponderados . . . . . . . . . . . . . . . 46

3.2.1 Ponderação na família Weibull . . . . . . . . . . . . . . . 46

3.2 .2 Ponderação no modelo semiparamétrico . . . . . . . . . . . . . . . . 48

3.3 Obtenção dos estimadores ponderados . . . . . . . . . . . . . 52 
4.1 Modelos Weibull . . . . . . . . . . . . . . . . . 57

4.2 Modelos semiparamétricos . . . . . . . . . . . . . . 63

4.2.1 Baixo percentual de omissão . . . . . . . . . . . . . . 63

4.2 .2 Médio percentual de omissão . . . . . . . . . . . . . . . . . . . 66

4.2 .3 Alto percentual de omissão . . . . . . . . . . . . . . . . 68

4.2.4 Comparação entre os cenários . . . . . . . . . . . . . . . 71

5 Considerações finais e estudos futuros $\quad 76$

$\begin{array}{ll}\text { A Derivadas de } 2^{a} \text { ordem } & 80\end{array}$

B Algumas considerações sobre as propriedades assintóticas $\quad 82$

B.1 Modelo Weibull . . . . . . . . . . . . . . . . . . . . . . . 82

B.1.1 Caso sem ponderação . . . . . . . . . . . . . . . 83

B.1.2 Família Weibull com ponderação . . . . . . . . . . . . . . 86

B.2 Modelo semiparamétrico . . . . . . . . . . . . . . . . . . 88

B.2.1 Modelo logístico para a probabilidade de cura . . . . . . . . . 89

B.2.2 Modelo de Cox para a sobrevivência dos não curados . . . . . . . . . 93

B.2.3 Distribuição assintótica do estimador ponderado no modelo de Cox . 103

$\begin{array}{ll}\text { C Aspectos Computacionais } & 110\end{array}$ 


\section{Capítulo 1}

\section{Introdução}

Em estudos que envolvem o acompanhamento de pacientes, freqüentemente há interesse em avaliar o efeito de fatores prognósticos sobre a sobrevida ou algum evento de interesse. Os modelos de regressão que relacionam tempos de sobrevivência e covariáveis são muito utilizados em estudos deste tipo, pois permitem analisar a influência simultânea dos fatores de interesse na evolução dos pacientes.

Esta tese trata de modelos para dados de sobrevivência na situação especial em que existem valores omissos nas covariáveis e que, além disso, há uma fração de pacientes não suscetíveis ao evento de interesse que está relacionada à omissão. Neste capítulo é feita uma introdução ao problema de dados omissos e ao conjunto de dados que motivou este trabalho. São descritas algumas características observadas nos dados e informações clínicas que evidenciam a presença de pacientes não suscetíveis ao evento de interesse. Ao final do capítulo, fazemos uma revisão bibliográfica com algumas propostas encontradas na literatura e descrevemos o conteúdo dos capítulos seguintes. 


\subsection{O problema de dados omissos}

Na prática, são comuns situações em que, por diversas razões, existem covariáveis com valores omissos para alguns e, freqüentemente, para muitos pacientes. Exemplos de situações que geram omissão incluem a recusa do paciente em fornecer alguma informação, perda de prontuários ou seu preenchimento incorreto, falha em análises bioquímicas, necessidade de exames invasivos ou de alto custo que não podem ser realizados em todos os pacientes e classificação indeterminada de sintomas ou doenças. No caso particular em que a realização de exames invasivos depende da condição do paciente, fica clara a relação entre omissão e pior prognóstico, pois os pacientes com omissão são os mais debilitados e sobrevivem menos que os pacientes sem omissão.

Quando covariáveis com dados omissos são incluídas nos modelos de regressão, os programas estatísticos usuais automaticamente excluem todos os indivíduos que apresentam pelo menos um dado omisso. Com isso, o procedimento tipicamente empregado por grande parte dos pesquisadores é utilizar apenas os sujeitos sem dados omissos, desconsiderando aqueles com observações incompletas, mesmo que isso aconteça em apenas algumas covariáveis. Além de descartar uma grande parte da informação disponível, está comprovado que a análise baseada apenas nos dados completos pode levar a estimadores altamente viesados e ineficientes, principalmente se a proporção de omissão for alta [Demissie, LaValley, Horton, Glynn \& Cupples (2003)]. Portanto, é importante desenvolver métodos que incorporem na análise informações sobre os casos incompletos.

\subsection{Motivação}

Nosso interesse em estudar dados omissos surgiu a partir de um conjunto de dados com informações de 1220 pacientes com insuficiência cardíaca grave encaminhados para o 
grupo de transplantes do Instituto do Coração - FMUSP.

A fim de avaliar os possíveis fatores de risco de óbito, foram registradas várias características demográficas, dados laboratoriais, dados de exames invasivos e não invasivos e de outras variáveis de interesse. O tempo da entrada no protocolo até o óbito, transplante (quando este ocorria) ou perda de acompanhamento (pelo encerramento da coleta das informações ou por outras causas) foi utilizado como resposta na análise dos dados.

A tabela abaixo apresenta algumas variáveis de interesse e a correspondente freqüência de dados omissos.

\begin{tabular}{lcc}
\hline variável & casos válidos (\%) & casos sem informação (\%) \\
\hline $\mathrm{Sx}$ & $1220(100)$ & - \\
$\mathrm{Fe}$ & $898(74)$ & $322(26)$ \\
Alt dop & $894(73)$ & $326(27)$ \\
$\mathrm{Na}$ & $679(56)$ & $541(44)$ \\
Indcar & $210(17)$ & $1010(83)$ \\
\hline Sx=sexo; Fe=fração de ejeção; Alt dop = alteração no doppler; Na = sódio sérico; Indcar=índice cardíaco.
\end{tabular}

Em uma primeira análise, Freitas, Chizzola, Paes, Lima \& Mansur (2005) ajustaram dois modelos de Cox para estes dados: um apenas com variáveis relacionadas a exames não invasivos (com 856 pacientes $(70 \%)$ da amostra total) e outro com variáveis de exames invasivos (com 194 pacientes, ou seja, apenas 16\% da amostra total). Apesar de a abordagem utilizada produzir modelos intuitivos e interpretativos, não foi utilizada toda a informação disponível para estimativas e testes. Procurou-se amenizar o impacto dos valores omissos ajustando os modelos invasivo e não invasivo separadamente, uma vez que o uso de um único modelo ocasionaria uma perda de informação ainda maior.

Como Freitas et al. (2005) utilizaram programas usuais, apenas os casos completos eram considerados no ajuste dos modelos. Portanto, a amostra que era de fato utilizada tornava-se cada vez menor à medida que variáveis com dados omissos eram incluídas, conforme se observa na tabela a seguir. 


\begin{tabular}{lc}
\hline variáveis incluídas no modelo & número de casos completos (\%) \\
\hline $\mathrm{Sx}$ & $1220(100)$ \\
$\mathrm{Sx}+\mathrm{Fe}$ & $898(74)$ \\
$\mathrm{Sx}+\mathrm{Fe}+$ Alt Dop & $820(67)$ \\
$\mathrm{Sx}+\mathrm{Fe}+\mathrm{Alt}$ dop $+\mathrm{Na}$ & $587(48)$ \\
$\mathrm{Sx}+\mathrm{Fe}+$ Alt dop $+\mathrm{Na}+$ Indcar & $123(10)$ \\
\hline Sx=sexo; Fe=fração de ejeção; Alt dop = alteração no doppler; $\mathrm{Na}=$ sódio sérico; Indcar=índice cardíaco.
\end{tabular}

Reanalisando os dados, observamos que, além da presença de dados omissos em muitas covariáveis de interesse, os pacientes com dados omissos apresentavam pior evolução quando comparados aos pacientes com dados completos (Figura 1).

\section{Relação entre sobrevivência e omissão}

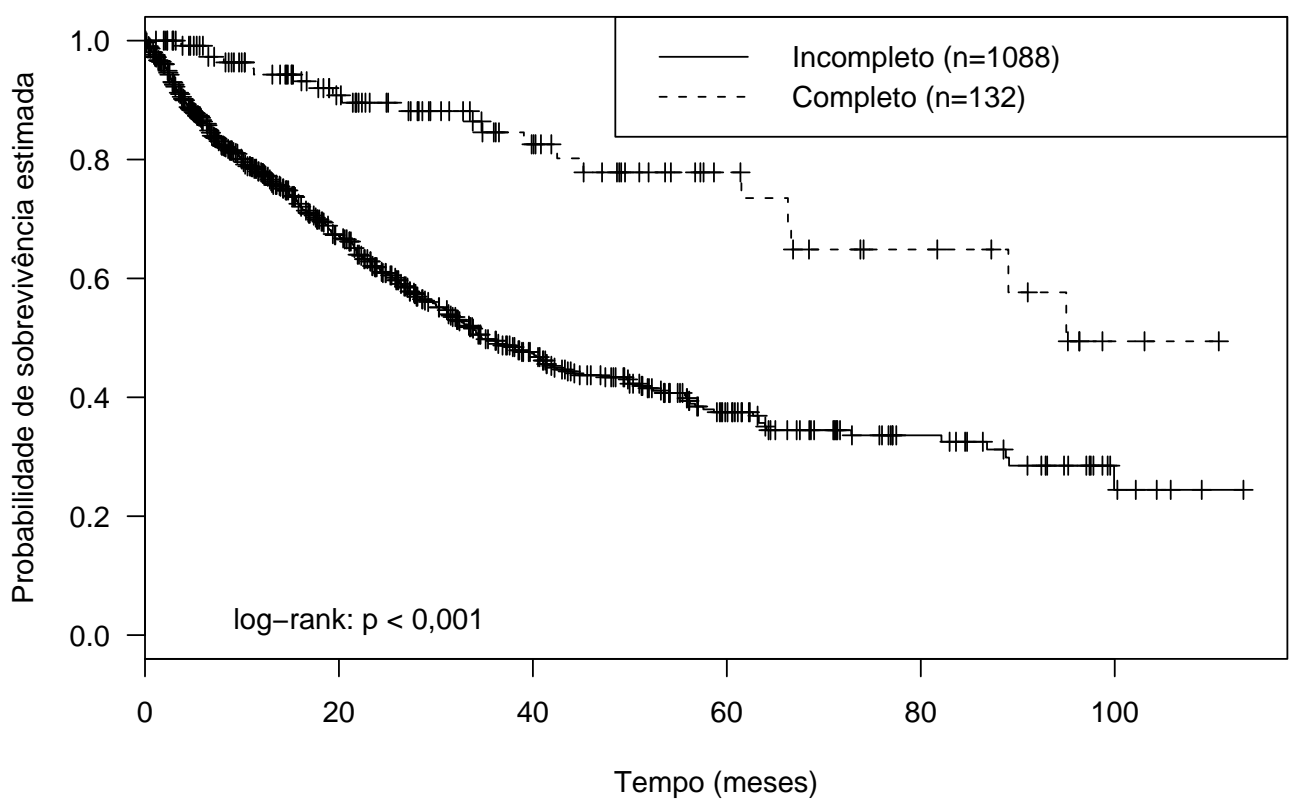

Figura 1: Estimativas de Kaplan-Meier para as proporções de sobreviventes em pacientes com dados completos e incompletos em pelo menos uma das seguintes covariáveis: fração de ejeção, alteração no dopler, sódio sérico e índice cardíaco. 
Além disso, quando comparamos subgrupos levando-se em conta a omissão segundo cada covariável, as diferenças entre as curvas de sobrevivência aumentavam ou diminuiam, dependendo da covariável em questão (Figura 2).

Fração de Ejeção

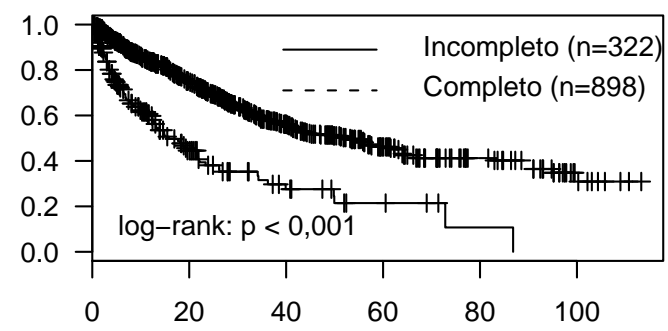

Alteração no Doppler

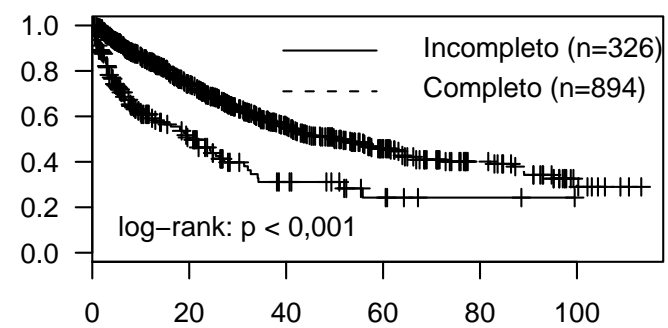

Sódio Sérico

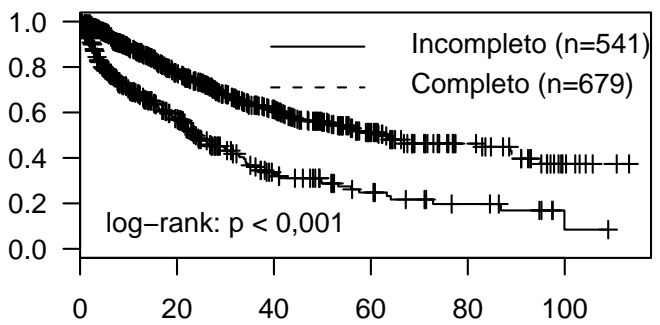

Índice Cardíaco

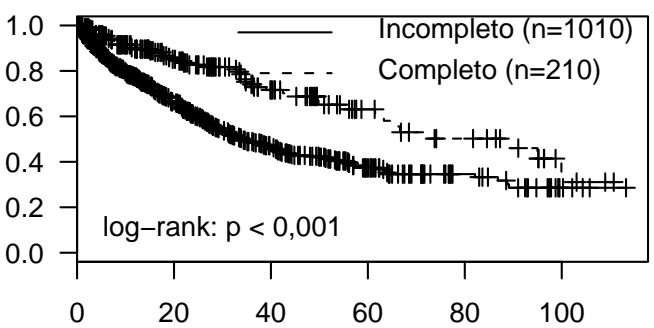

Figura 2: Estimativas de Kaplan-Meier para as proporções de sobreviventes em pacientes com dados completos e incompletos para algumas covariáveis de interesse.

As Figuras 1 e 2 mostram claramente que a suposição de omissão não informativa não é razoável para este conjunto de dados. O fato de a omissão estar relacionada à pior evolução e a algumas covariáveis não foi utilizado nos modelos ajustados em Freitas et al. (2005).

É conveniente notar que, enquanto aguardavam na fila de transplantes, os pacientes eram tratados e que, segundo os pesquisadores, alguns deles respondiam bem 
ao tratamento e apresentavam prognóstico melhor que o esperado para pacientes com insuficiência cardíaca grave que necessitavam ter o coração transplantado. Como conseqüência, mais de $60 \%$ das observações eram censuradas.

O alto percentual de censuras pode ser um indício da presença de curados (Maller \& Zhou (1996)), ou seja, pacientes não suscetíveis ao evento de interesse. Devido à presença de muitas censuras, ocasionadas pela boa resposta ao tratamento, é razoável supor a presença de curados nesse conjunto de dados. Como existe uma relação direta entre a cura e a sobrevida (quanto maior a proporção de curados maior o tempo de sobrevivência), é provável que as diferenças entre os subgrupos observados na Figura 2 sejam devidas a diferentes proporções de curados e não a taxas de falhas distintas.

A partir deste problema surgiu o interesse em se investigar formas para analisar dados com proporção de curados e omissão nas covariáveis, buscando alternativas para aproveitar os dados disponíveis dos pacientes com valores omissos, que até então estavam sendo totalmente desconsiderados.

\subsection{Mecanismos de omissão}

A análise de dados com omissão deve levar em conta o processo segundo o qual as omissões acontecem, conhecido como mecanismo de omissão [Little \& Rubin (2002)]. Intuitivamente, pode-se dizer que, quando a subamostra de dados completos apresenta o mesmo perfil da amostra original, a análise que considera apenas as observações completas é adequada. Porém, se os indivíduos com dados omissos, quando comparados aos indivíduos com dados completos, apresentam diferenças significativas quanto às covariáveis e à resposta de interesse, a perda de informação pode ser grande, pois os casos excluídos podem conter informação relevante sobre os parâmetros de interesse.

Em dados de sobrevivência, supor que os indivíduos com dados omissos têm o 
mesmo perfil dos indivíduos com dados completos significa supor que a probabilidade de omissão não depende das covariáveis nem dos tempos de falha ou censura. Esta suposição é razoável se, por exemplo, a omissão ocorre porque o paciente muda de cidade ou entra tardiamente no estudo sem realizar todos os exames antes do término do período de observação. Nesse caso, a omissão é chamada de completamente aleatória (missing completely at random - MCAR) e não exerce influência sobre as estimativas de interesse (omissão não informativa).

Outra suposição menos restritiva é que a distribuição dos dados omissos depende apenas do que é observado, ou seja, das covariáveis com dados completos e dos tempos observados de falha ou censura. Por exemplo, nos dados sobre insuficiência cardíaca analisados neste trabalho, dados omissos foram observados em pacientes chagásicos que morreram antes da realização dos exames. Pode-se supor, neste caso, que a omissão está associada apenas à etiologia chagásica e aos menores tempos de falha, variáveis observadas para todos os indivíduos. Neste caso, a omissão é chamada de aleatória (missing at random - MAR).

Nos dois casos anteriores (MAR e MCAR), o mecanismo de omissão é ignorável, pois a probabilidade da observação ser omissa não depende de qualquer quantidade não observada. Quando a omissão depende também de covariáveis com dados omissos e não pode ser completamente explicada pelos dados observados, a omissão é chamada de não aleatória (missing not at random - MNAR ) ou não ignorável.

Na notação usualmente empregada em análise de sobrevivência, considera-se que $\left\{T_{i}, C_{i}, \mathbf{Z}_{\mathbf{i}}, i=1, \ldots, n\right\}$ são observações independentes em que $T_{i}$ são os tempos de falha, $C_{i}$ são os tempos de censura e $\mathbf{Z}_{i}$ são os vetores $p$ x 1 de covariáveis associadas ao indivíduo $i$. Para situações em que há dados omissos, pode-se particionar $\mathbf{Z}_{i}$ em $\left\{\mathbf{Z}_{i, \text { comp }}, \mathbf{Z}_{i, \text { incomp }}\right\}$ em que $\mathbf{Z}_{i, \text { comp }}$ é o vetor de covariáveis completamente observadas e $\mathbf{Z}_{i, \text { incomp }}$ é o vetor de covariáveis com dados omissos. 
Definindo, para a $j$-ésima covariável, $j=1, \ldots, p$, um indicador de omissão $R_{i j}$ que vale 1 se $Z_{i j}$ é observada e 0 em caso contrário e utilizando o símbolo $\perp$ para indicar independência ${ }^{1}$, os 3 mecanismos de omissão podem ser representados por:

- MCAR: $R_{i j}(t) \perp\left\{\mathbf{Z}_{i, c o m p}, \mathbf{Z}_{i, i n c o m p}, X_{i}, \Delta_{i}\right\} / X_{i} \geq t, \quad \forall j=1, \ldots, p$,

- MAR: $R_{i j}(t) \perp\left\{\mathbf{Z}_{i, \text { incomp }}\right\} / X_{i}, \Delta_{i}, X_{i} \geq t, \mathbf{Z}_{i, \text { comp }}, \quad \forall j=1, \ldots, p$,

- MNAR: $R_{i j}(t) \not \perp\left\{\mathbf{Z}_{i, \text { comp }}, \mathbf{Z}_{i, \text { incomp }}, X_{i}, \Delta_{i}\right\} / X_{i} \geq t, \quad \forall j=1, \ldots, p$.

em que $X_{i}=\min \left\{T_{i}, C_{i}\right\}$ e $\Delta_{i}=I_{\left(T_{i} \leq C_{i}\right)}, i=1, \ldots, n$.

O procedimento que consiste em analisar apenas os dados completos é conhecido na literatura como Análise de Casos Completos ou ACC (Complete Case Analysis - CCA) e apesar de ser a técnica mais freqüentemente utilizada, pressupõe que a omissão é do tipo completamente aleatória (MCAR), o que não é razoável na maioria das situações práticas.

\subsection{Revisão Bibliográfica}

A maior parte dos artigos que tratam de dados omissos considera a omissão na resposta e não nas covariáveis. Muitos deles são relacionados com dados longitudinais, cuja omissão na resposta ocorre em um ou mais momentos de avaliação [Diggle \& Kenward (1994), Folmann \& Wu (1995), Wu \& Follmann (1999)].

Os primeiros artigos que consideram omissão nas covariáveis estão no contexto de modelos lineares generalizados (MLG) e, em especial, de regressão logística (Vach 1994). Alguns desses artigos consideram omissão ignorável (MAR ou MCAR), dentre os quais destacamos Gibbons \& Hosmer (1991) em regressão logística e Lipsitz, Parzen

\footnotetext{
${ }^{1}$ Isto é, $X \perp Y \mid Z$ significa que $X$ e $Y$ são independentes, condicional a qualquer valor de $Z$
} 
\& Ewell (1998) em regressão logística condicional. Blackhurst \& Schluchter (1989) consideram também omissão não ignorável e Horton \& Laird (1998) tratam o caso em que a omissão depende de uma variável auxiliar que não faz parte do modelo de regressão.

Sob o enfoque mais geral de MLG com omissão ignorável nas covariáveis foram encontradas basicamente duas abordagens: uma baseada em máxima verossimilhança usando o algoritmo EM [Ibrahim (1990), Little \& Schluchter (1985)] e outra em equações de estimação ponderadas [Robins, Rotnitzky \& Zhao (1994), Zhao, Lipsitz \& Lew (1996)].

Ibrahim (1990) examina a classe MLG quando há omissão aleatória (MAR) e as covariáveis são discretas. Ele utiliza o algoritmo EM para obter os estimadores de máxima verossimilhança dos parâmetros do modelo e mostra que o passo E para qualquer MLG pode ser expresso como uma ponderação da verossimilhança para dados completos. Esta metodologia, originalmente proposta por Little \& Schluchter (1985) para dados com distribuição Normal, ficou conhecida na literatura como método dos pesos e deu origem a algumas extensões para dados longitudinais, modelos de sobrevivência paramétricos e semiparamétricos. Ibrahim, Chen \& Lipsitz (1999), por exemplo, investigam situações que incluem, além de variáveis categorizadas, variáveis contínuas e utilizam uma adaptação da versão Monte Carlo do algorimo EM para obter as estimativas de máxima verossimilhança. Horton \& Laird (1998) descrevem o método de pesos em detalhes e, por meio de exemplos, ilustram sua aplicação discutindo vantagens e limitações.

Sob a abordagem de equações de estimação ponderadas, Robins et al. (1994) propõem uma classe de estimadores semiparamétricos baseados em equações de estimação ponderadas pelo inverso da probabilidade de o dado ser observado (inverse probability weighted estimating equations). Eles são consistentes sob a suposição MAR e quando 
as probabilidades de omissão são conhecidas ou podem ser modeladas parametricamente. Buscando relacionar esta proposta com o método de pesos, Lipsitz, Ibrahim \& Zhao (1999) sugerem um algoritmo EM para resolver equações de estimação ponderadas, obtendo propriedades de forma semelhantes às de máxima verossimilhança, e comparam as duas abordagens através de simulações e estudos assintóticos.

Em um artigo mais recente, Ibrahim, Chen, Lipsitz \& Herring (2005) fazem uma revisão dos métodos para analisar dados omissos em MLG. Além do método dos pesos e de equações ponderadas, eles consideram ainda métodos de imputação múltipla e métodos bayesianos completos. Na imputação múltipla, para cada dado omisso, considera-se um conjunto de valores plausíveis que representam a incerteza sobre o verdadeiro valor a ser imputado. A idéia é criar múltiplos conjuntos de dados completos, imputando valores plausíveis onde há omissão, analisar cada conjunto como se os dados fossem completos e então combinar as inferências em um único procedimento. Os métodos bayesianos envolvem a especificação de prioris para todos os parâmetros bem como a especificação das distribuições das covariáveis com omissão, que são amostradas a partir da distribuição condicional via amostrador de Gibbs. Maiores detalhes sobre métodos bayesianos são encontrados em Ibrahim, Chen \& Lipsitz (2002).

No contexto de análise de sobrevivência, os primeiros autores que tratam explicitamente o problema de omissão nas covariáveis são Schluchter \& Jackson (1989). Sua proposta é estender a metodologia de modelos log-lineares para análise de sobrevivência no caso em que há covariáveis categorizadas com omissão ignorável. O método consiste em assumir um modelo log-linear para o risco e um modelo multinomial para as probabilidades em uma tabela de contingência definida pelas covariáveis categorizadas. Eles consideram os algoritmos EM e Newton-Raphson na estimação dos parâmetros do modelo e obtêm suas propriedades assintóticas. Além da suposição MAR, o método requer que os tempos de censura não dependam de qualquer covariável com omissão. 
Baker (1994) propôs uma extensão deste modelo para o caso com omissão não ignorável considerando dados agrupados, porém ainda supondo covariáveis categorizadas.

Sob a suposição MAR e considerando covariáveis categorizadas, Lipsitz \& Ibrahim (1996a) sugerem uma extensão do método dos pesos de Ibrahim (1990) para modelos de sobrevivência paramétricos com distribuição Weibull e Log-normal.

Para acomodar covariáveis com dados omissos em modelos semiparamétricos, inicialmente foram propostas modificações na função de verossimilhança parcial e métodos de imputação. Schemper \& Smith (1990) discutem a situação em que se deseja testar os efeitos de tratamentos ajustados por covariáveis dicotômicas, das quais algumas são observações com omissão. A idéia é utilizar imputação de dados, substituindo os dados omissos pelas médias dos dados completos, calculadas e imputadas separadamente para cada tratamento. A metodologia é ilustrada por um estudo de pacientes com câncer no qual uma importante covariável apresenta dados omissos. Apesar de sua simplicidade, o método é inválido quando a omissão não é aleatória e as covariáveis com dados omissos estão associadas aos tratamentos.

O estimador de máxima verossimilhança parcial aproximada (Approximate Partial Likelihood Estimator - APLE), proposto por Lin \& Ying (1993), é um dos primeiros métodos a sugerir modificações na função de verossimilhança parcial (Cox (1975)). Com base no método de pseudo-escore de Self \& Prentice (1986), desenvolvido para estudos de caso-coorte, Lin \& Ying (1993) sugerem substituir o termo da média ponderada empírica no tempo $t$ da função escore obtida a partir da verossimilhança parcial de Cox, por uma estimativa baseada apenas nos indivíduos que estão em risco e têm dados completos para todas as covariáveis no tempo $t$. Introduzindo indicadores de omissão na função escore, as observações incompletas também são consideradas na estimação dos parâmetros. A função escore modificada pode ser utilizada mesmo quando 
o padrão de omissão não é monótono ${ }^{2}$, porém, é necessário supor que a omissão seja completamente aleatória (MCAR). Portanto, embora o estimador tenha um bom desempenho na comparação com a ACC, ele perde eficiência e torna-se viesado quando o mecanismo de omissão não é MCAR (Paik \& Tsai 1997).

Em outra abordagem, Zhou \& Pepe (1995) apresentam métodos para lidar com a omissão quando há um bloco de covariáveis com omissão. O estimador proposto por Zhou \& Pepe (1995), conhecido como Estimador de Verossimilhança Parcial Estimada (Estimated Partial Likelihood Estimator - EPLE), requer informações sobre dados de covariáveis auxiliares e uma amostra de validação com dados completos em todas as covariáveis.

Sob o modelo de Cox, o estimador baseado em imputação de dados mais conhecido foi proposto por Paik \& Tsai (1997) e é denominado Estimador de Máxima Verossimilhança Parcial Imputada (Imputed Parcial Likelihood Estimator - IPLE). O método consiste em imputar os valores omissos na equação escore da verossimilhança parcial e requer que algumas covariáveis sejam completamente observadas para todos os indivíduos do estudo. Uma de suas principais limitações é a dificuldade de implementação computacional, o que faz com que ele seja muito pouco utilizado na prática.

Lipsitz \& Ibrahim (1998) propõem um conjunto de equações de estimação para estimar os parâmetros do modelo de Cox quando há omissão em covariáveis categorizadas, sob a suposição MAR. Eles usam o algoritmo EM via Monte Carlo para resolver essas equações, obtendo também as variâncias assintóticas. Segundo os autores, este método pode ser considerado uma extensão dos métodos de Schluchter \& Jackson (1989), Ibrahim (1990) e Lipsitz \& Ibrahim (1996b) para o modelo semiparamétrico

\footnotetext{
${ }^{2}$ Se as covariáveis são ordenadas da maior para menor porcentagem de dados omissos, considerase que o padrão de omissão é monótono quando os sujeitos observados na primeira variável são um subconjunto do grupo de sujeitos que são observados na segunda variável, aqueles observados na segunda são um subconjunto da terceira e assim por diante (Little \& Rubin (2002),pag.7).
} 
de Cox. Leong, Lipsitz \& Ibrahim (2001) estenderam o método proposto por Lipsitz \& Ibrahim (1998) para o caso de omissão não ignorável, mas além de considerar apenas covariáveis binárias, o método não pode ser estendido para variáveis contínuas. A extensão proposta por Herring \& Ibrahim (2001) utiliza uma aproximação diferente e usa um algoritmo EM ponderado que permite omissão em covariáveis categorizadas e contínuas.

O método proposto por Chen \& Little (1999), chamado por eles de não paramétrico, utiliza o algoritmo EM para estimar os parâmetros do modelo de Cox com omissão nas covariáveis, assumindo que a omissão é aleatória (MAR). O método é direto quando as covariáveis são categorizadas; no entanto, para covariáveis contínuas, a estimação no passo E é mais complicada, pois envolve técnicas numéricas de integração. Chen (2002) melhorou o método de Chen \& Little (1999) relaxando algumas suposições, porém, considera que a omissão ocorre apenas nas covariáveis discretas.

Paralelamente ao trabalho de Chen \& Little (1999), Martinussen (1999) propõe outra extensão do método de Ibrahim (1990) para modelos de Cox com omissão nas covariáveis. Usando algoritmo EM, ele especifica um modelo completo deixando as covariáveis incompletas como aleatórias e então maximiza, em cada iteração, uma verossimilhança perfilada, dadas as estimativas correntes e os dados observados. Neste caso assume-se que as covariáveis com omissão são categorizadas. Se forem contínuas, as somas especificadas no passo E são substituídas por integrais e métodos numéricos de integração bem como outras aproximações devem ser considerados.

Entre os demais artigos que analisam dados de sobrevivência com omissão nas covariáveis, citamos Herring, Ibrahim \& Lipsitz (2004) para modelos de Cox com omissão não ignorável e Herring, Ibrahim \& Lipsitz (2002) que tratam a omissão em modelos de fragilidade. Ambos são métodos bayesianos que utilizam a versão Monte Carlo do algoritmo EM e Amostrador de Gibbs. Não foram encontrados na literatura métodos 
freqüentistas que tratam a omissão não ignorável, considerando covariáveis categorizadas e contínuas.

Métodos para análise de dados com proporções de curados têm sido objeto de estudo de muitos artigos recentes (Zhao \& Zhou (2006), Fang, Li \& Sun (2005), Lam \& Xue (2005), Li \& Feng (2005), Yin \& Ibrahim (2005a), Yin \& Ibrahim (2005b)). Apesar disso, poucos foram os artigos encontrados que consideram omissão nas covariáveis. Os artigos que tratam de omissão em dados com fração de curados em geral utilizam métodos bayesianos, alguns computacionalmente intensivos (Cho, Schenker, Taylor \& Zhuang (2001), Chen \& Ibrahim (2001), Chen, Ibrahim \& Lipsitz (2002), Gagnon, Glickman, Myers \& Cupples (2003)). Até o momento não foram encontradas propostas clássicas para lidar com dados com fração de curados quando há omissão nas covariáveis de interesse.

\subsection{Proposta do trabalho}

Uma das maneiras de lidar com dados omissos é ponderar as observações completas com o intuito de compensar grupos que eventualmente estejam sub-representados e assim corrigir parte do viés de seleção (Therneau \& Grambsch 2000). Como o interesse é analisar dados que apresentam uma proporção substancial de curados, a proposta deste trabalho é utilizar modelos com fração de cura inserindo ponderações que levem em conta a omissão nas covariáveis e sua relação com piores prognósticos. Neste trabalho são consideradas duas alternativas para os pesos, uma baseada nas probabilidades de omissão e outra baseada nas probabilidades de cura. Apesar de os modelos finais considerarem apenas os casos completos, os métodos propostos utilizam parte da informação contida nas observações incompletas, o que normalmente é desperdiçado na análise de casos completos. 
Este trabalho está organizado da seguinte forma: o Capítulo 2 traz uma introdução aos modelos com fração de cura e uma descrição do processo de estimação geralmente usado para modelos Weibull e semiparamétrico de Cox. Os modelos com fração de cura ponderados, bem como as propostas para obtenção dos pesos, são descritos no Capítulo 3. O Capítulo 4 ilustra a aplicação dos métodos através de simulações no conjunto de dados de insuficiência cardíaca descrito anteriormente. Considerações finais e perspectivas de trabalhos futuros são discutidos no Capítulo 5. 


\section{Capítulo 2}

\section{Modelos com fração de cura}

Em muitos estudos de acompanhamento, pacientes que respondem bem ao tratamento podem tornar-se imunes aos sinais e sintomas da doença e assim serem considerados curados, ou seja, não suscetíveis ao evento de interesse. Neste caso, é importante estudar a proporção de pacientes que permanecem curados e analisar os efeitos das covariáveis, não só sobre o tempo de sobrevivência, como também sobre a taxa (ou fração) de cura.

Modelos para análise de dados com proporção de curados são freqüentemente chamados de modelos com fração de cura. Neste capítulo, é feita uma introdução aos principais conceitos relacionados a esses modelos e uma descrição dos processos de estimação dos parâmetros dos modelos utilizados neste trabalho.

Consideramos uma população heterogênea que pode ser dividida em duas subpopulações: imunes (curados) e suscetíveis (não curados). Em um estudo experimental analisado por Pierce, Stewart \& Kopecky (1979), por exemplo, os autores observaram que havia uma proporção substancial de animais expostos a toxinas que continuavam vivos ao final do experimento. Segundo eles, não fazia sentido considerar tais animais como observações censuradas vindas da mesma população daqueles que haviam morrido. Devido a essa heterogeneidade, é natural considerar um modelo construído a 
partir da mistura de duas distribuições: uma representando a distribuição dos tempos de falha ou sobrevivência dos não curados e outra correspondendo a uma distribuição degenerada que permita tempos de sobrevida (em princípio, infinitos) para pacientes curados (Maller \& Zhou (1996)).

A idéia de utilizar modelos de mistura na análise de dados de sobrevivência com proporção de curados não é recente (Boag (1949), Berkson \& Gage (1952)). Desde então, algumas alternativas têm sido sugeridas baseadas em métodos paramétricos e não paramétricos e nas abordagens clássicas e bayesianas.

Este trabalho concentra-se em dois tipos de modelos de mistura: um paramétrico, supondo a família Weibull, e outro semiparamétrico, baseado no modelo de Cox. A seguir, a formulação usualmente empregada em modelos com fração de cura é apresentada. O termo "sobreviventes de longa duração" (long-term survivors) é comumente utilizado para denominar indivíduos não suscetíveis ao evento, porém, por convenção, esses indivíduos serão sempre referidos como curados .

\subsection{Formulação geral}

Seja $T$ uma variável aleatória que representa o tempo até o evento de interesse e sejam $f_{C}(t)$ e $S_{C}(t)$ a função densidade de probabilidade e a função de sobrevivência condicionais a pacientes curados. Como os indivíduos curados (C) não apresentarão o evento de interesse, define-se seu tempo de falha como infinito. Consequentemente,

$$
S_{C}(t)=P(T>t \mid C)=1 \quad \forall t<\infty .
$$

Ou seja, em pacientes curados, a função de sobrevivência é igual a 1 para todo $t \geq 0$. 
Em uma população com fração de curados, um indivíduo sobrevive até o tempo $t$ se ele for curado ou se ele for não curado, mas com tempo de falha superior a $t$. Portanto, denotando por $\pi$ a probabilidade de não cura, a função de sobrevivência (não condicional) pode ser escrita como

$$
S(t)=(1-\pi) S_{C}(t)+\pi S_{N C}(t) \Rightarrow S(t)=(1-\pi)+\pi S_{N C}(t)
$$

isto é,

$$
f(t)=\pi f_{N C}(t)
$$

em que $f_{N C}(t)$ e $S_{N C}(t)$ são a função densidade de probabilidade e a função de sobrevivência, condicionais a pacientes não curados.

Note que, quando $t \rightarrow \infty, S_{N C}(t) \rightarrow 0$ e $S(t) \rightarrow 1-\pi$, isto é, a fração de cura corresponde ao limite da função de sobrevivência não condicional, que é imprópria.

Em modelos de sobrevivência, as falhas e as censuras são representadas na função de verossimilhança, respectivamente, pela função densidade e pela função de sobrevivência. Logo, um indivíduo que falha em $t_{i}$ contribui com $f\left(t_{i}\right)=\pi_{i} f_{N C}\left(t_{i}\right)$ e a contribuição de um indivíduo que está em risco no tempo $t_{i}$ é $S\left(t_{i}\right)=\left(1-\pi_{i}\right)+\pi_{i} S_{N C}\left(t_{i}\right)$. Portanto, se $\boldsymbol{\theta}$ é o parâmetro de interesse e $\Delta_{i}$ é o indicador de falha do indivíduo $i$, a forma geral da função de verossimilhança para modelos com fração de cura é

$$
\begin{aligned}
L_{n}(\boldsymbol{\theta}) & =\prod_{i=1}^{n}\left[f\left(t_{i}\right)\right]^{\Delta_{i}}\left[S\left(t_{i}\right)\right]^{1-\Delta_{i}} \\
& =\prod_{i=1}^{n}\left[\pi_{i} f_{N C}\left(t_{i}\right)\right]^{\Delta_{i}}\left[1-\pi_{i}+\pi_{i} S_{N C}\left(t_{i}\right)\right]^{1-\Delta_{i}}
\end{aligned}
$$

Note que a probabilidade de não cura $\left(\pi_{i}\right)$ pode variar de indivíduo para indivíduo, pois é razoável assumir que pode depender de características individuais a serem representadas por covariáveis. Nos modelos de mistura para dados com fração de curados, 
o efeito de covariáveis na probabilidade de cura é naturalmente especificado por um modelo logístico.

No termo de sobrevivência, a distribuição dos tempos de sobrevivência também pode variar entre os indivíduos e assim os parâmetros são especificados em função de covariáveis de interesse. Nesse contexto, têm sido utilizados modelos paramétricos da família Weibull (Ghitany, Maller \& Zhou (1994), Farewell (1982), Ng, McLachlan, Yau \& Lee (2004)), Lognormal e Gama (Peng, Dear \& Denham (1998)) ou modelos semiparamétricos (Kuk \& Chen (1992), Sy \& Taylor (2000), Peng (2003b), Lu \& Ying (2004) e Zhao \& Zhou (2006)). Neste trabalho, para a abordagem paramétrica, escolhemos a família Weibull por ser a mais frequentemente utilizada. Pela mesma razão, o modelo de Cox foi escolhido para a abordagem semiparamétrica.

\subsection{Família Weibull com fração de cura}

No modelo da família Weibull com fração de cura, assume-se que os tempos de falha dos indivíduos não curados seguem uma distribuição $W$ eibull $\left(\lambda_{i}, \delta\right), \operatorname{com} \lambda_{i}>0 \mathrm{e}$ $\delta>0, i=1, \ldots, n$. Neste modelo, a função densidade e a função de sobrevivência condicionais a pacientes não curados são dadas por

$$
f_{N C}\left(t_{i}\right)=\lambda_{i} \delta\left(\lambda_{i} t_{i}\right)^{\delta-1} \mathrm{e}^{-\left(\lambda_{i} t_{i}\right)^{\delta}} \quad \text { e } \quad S_{N C}\left(t_{i}\right)=\mathrm{e}^{-\left(\lambda_{i} t_{i}\right)^{\delta}}, \quad i=1, \ldots, n .
$$

Substituindo em (2.1), a função de verossimilhança é dada por

$$
L_{n}(\boldsymbol{\theta})=\prod_{i=1}^{n}\left(\pi_{i} \lambda_{i} \delta\left(\lambda_{i} t_{i}\right)^{\delta-1} \mathrm{e}^{-\left(\lambda_{i} t_{i}\right)^{\delta}}\right)^{\Delta_{i}}\left(1-\pi_{i}+\pi_{i} \mathrm{e}^{-\left(\lambda_{i} t_{i}\right)^{\delta}}\right)^{1-\Delta_{i}}
$$

É importante notar que os termos $\lambda_{i}$ e $\pi_{i}$, devem ser parametrizados adequadamente a fim de se incluir o efeito de potenciais fatores prognósticos nos tempos de falha e 
probabilidades de cura, respectivamente. Além disso, a parametrização utilizada deve evitar, sempre que possível, a ocorrência de problemas de fronteira.

Este modelo pode ser facilmente generalizado para permitir a inclusão de covariáveis no parâmetro de forma $\delta$. Neste caso, pode-se utilizar uma função de ligação loglinear que faz com que cada $\delta_{i}$ seja estritamente positivo (Maller \& Zhou (1996)). Neste trabalho considerou-se o caso em que $\delta$ não varia entre indivíduos, e o que consequentemente tem interpretação mais simples. Portanto, no processo de estimação, o interesse é obter os estimadores do escalar $\delta$ e de dois vetores de parâmetros: um associado à probabilidade de não cura $\left(\pi_{i}\right)$ e outro relacionado à distribuição dos tempos de sobrevivência $\left(\lambda_{i}\right)$.

\subsubsection{Estimação dos parâmetros}

Sob a parametrização considerada neste trabalho, o termo $\lambda_{i}$ depende de um vetor $\left(r_{1}+1\right) \times 1$ de covariáveis $\mathbf{Z}_{1 i}=\left(Z_{0,1 i}=1, Z_{1,1 i}, \ldots, Z_{r_{1}, 1 i}\right)$ através da função

$$
\lambda_{i}=\mathrm{e}^{\mathbf{Z}_{1 i}^{\prime} \boldsymbol{\beta}}, \quad \boldsymbol{\beta} \in \mathbb{R}^{r_{1}+1}
$$

na qual $\boldsymbol{\beta}$ é um vetor $\left(r_{1}+1\right)$ x 1 de parâmetros desconhecidos.

Por outro lado, assume-se que a probabilidade de não cura $\pi_{i}$ depende de um vetor $\left(r_{2}+1\right) \times 1$ de covariáveis $\mathbf{Z}_{2 i}=\left(Z_{0,2 i}=1, Z_{1,2 i}, \ldots, Z_{r_{2}, 2 i}\right)$ por meio do modelo logístico

$$
\pi_{i}=\frac{\mathrm{e}^{\left(\boldsymbol{Z}_{2 i}^{\prime} \boldsymbol{\alpha}\right)}}{1+\mathrm{e}^{\left(\boldsymbol{Z}_{2 i}^{\prime} \boldsymbol{\alpha}\right)}}, \quad \boldsymbol{\alpha} \in \mathbb{R}^{r_{2}+1}
$$

em que $\boldsymbol{\alpha}$ é um vetor de parâmetros desconhecidos de dimensão $\left(r_{2}+1\right)$ x 1 .

Convém observar que pode haver intersecção ou até coincidência dos conjuntos das covariáveis contidas em $\boldsymbol{Z}_{1 i}$ e $\boldsymbol{Z}_{2 i}$, ou seja, covariáveis possivelmente relacionadas à 
sobrevivência também podem estar relacionadas à fração de cura.

Tomando o logaritmo da verossimilhança (2.2), tem-se que

$$
\begin{aligned}
\log L_{n}(\boldsymbol{\theta})= & \sum{ }_{i=1}^{n} \Delta_{i}\left(\log \pi_{i}+\log \delta+\delta \log \lambda_{i}+(\delta-1) \log t_{i}-\left(\lambda_{i} t_{i}\right)^{\delta}\right) \\
& +\quad\left(1-\Delta_{i}\right) \log \left(1-\pi_{i}+\pi_{i} \mathrm{e}^{-\left(\lambda_{i} t_{i}\right)^{\delta}}\right) .
\end{aligned}
$$

As derivadas do logaritmo de $L_{n}(\boldsymbol{\theta})$ em relação aos parâmetros do modelo logístico $(\boldsymbol{\alpha})$ e do modelo Weibull ( $\boldsymbol{\beta}$ e $\delta$ ), obtemos a função escore

$$
\begin{aligned}
\mathbf{U}_{n}(\boldsymbol{\theta}) & =\frac{\partial \log L_{n}(\boldsymbol{\theta})}{\partial \boldsymbol{\theta}}=\left(\begin{array}{c}
\frac{\partial \log L_{n}(\boldsymbol{\theta})}{\partial \boldsymbol{\beta}} \\
\frac{\partial \log L_{n}(\boldsymbol{\theta})}{\partial \boldsymbol{\alpha}} \\
\frac{\partial \log L_{n}(\boldsymbol{\theta})}{\partial \delta}
\end{array}\right)_{r \times 1} \\
& =\sum_{i=1}^{n}\left(\begin{array}{ccc}
\mathbf{Z}_{1 i} & 0 & 0 \\
0 & \mathbf{Z}_{2 i} & 0 \\
0 & 0 & 1
\end{array}\right)_{r \times 3}\left(\begin{array}{c}
s_{i 1}(\boldsymbol{\theta}) \\
s_{i 2}(\boldsymbol{\theta}) \\
s_{i 3}(\boldsymbol{\theta})
\end{array}\right)_{3 \times 1}
\end{aligned}
$$

em que $r=r_{1}+r_{2}+3 \mathrm{e}$

$$
\begin{gathered}
s_{i 1}(\boldsymbol{\theta})=\Delta_{i}\left(\delta-\delta\left(\lambda_{i} t_{i}\right)^{\delta}\right)-\left(1-\Delta_{i}\right)\left(\frac{\pi_{i}\left(\lambda_{i} t_{i}\right)^{\delta} \delta \mathrm{e}^{-\left(\lambda_{i} t_{i}\right)^{\delta}}}{1-\pi_{i}\left(1-\mathrm{e}^{-\left(\lambda_{i} t_{i}\right)^{\delta}}\right)}\right), \\
s_{i 2}(\boldsymbol{\theta})=\Delta_{i}\left(1-\pi_{i}\right)+\left(1-\Delta_{i}\right)\left(\frac{-\pi_{i}\left(1-\pi_{i}\right)\left(1-\mathrm{e}^{-\left(\lambda_{i} t_{i}\right)^{\delta}}\right)}{1-\pi_{i}\left(1-\mathrm{e}^{-\left(\lambda_{i} t_{i}\right)^{\delta}}\right)}\right), \\
\text { e } s_{i 3}(\boldsymbol{\theta})=\Delta_{i}\left(\frac{1}{\delta}+\log \lambda_{i}+\log t_{i}-\left(\lambda_{i} t_{i}\right)^{\delta} \log \left(\lambda_{i} t_{i}\right)\right)+\left(1-\Delta_{i}\right)\left(\frac{\pi_{i}\left(\lambda_{i} t_{i}\right)^{\delta} \log \left(\lambda_{i} t_{i}\right) \mathrm{e}^{-\left(\lambda_{i} t_{i}\right)^{\delta}}}{1-\pi_{i}\left(1-\mathrm{e}^{-\left(\lambda_{i} t_{i}\right)^{\delta}}\right)}\right) .
\end{gathered}
$$


Note que, para cada indivíduo, constrói-se uma matriz de covariáveis, e não um vetor de covariáveis como nos modelos tradicionais, sem curados.

O estimador de máxima verossimilhança de $(\boldsymbol{\alpha}, \boldsymbol{\beta}, \delta)$ para o modelo Weibull com fração de cura é obtido solucionando a equação

$$
\mathbf{U}_{n}(\boldsymbol{\theta})=\sum_{i=1}^{n} \mathbf{Z}_{i} \mathbf{s}_{i}(\boldsymbol{\theta})=\mathbf{0}
$$

em que $\mathbf{Z}_{i}$ denota a matriz não estocástica dada por $\left(\begin{array}{ccc}\mathbf{Z}_{1 i} & 0 & 0 \\ 0 & \mathbf{Z}_{2 i} & 0 \\ 0 & 0 & 1\end{array}\right)_{r \times 3}$ e $\mathbf{s}_{i}(\boldsymbol{\theta})=\left(s_{i 1}(\boldsymbol{\theta}), s_{i 2}(\boldsymbol{\theta}), s_{i 3}(\boldsymbol{\theta})\right)^{\prime}$.

Como não existe forma explícita para os estimadores, é preciso recorrer a métodos iterativos e encontrá-los numericamente. Com a inversa da matriz de segundas derivadas parciais do logaritmo da verossimilhança, obtém-se uma estimativa da matriz de covariância assintótica dos estimadores dos parâmetros. Algumas propriedades assintóticas desses estimadores serão discutidas brevemente no Capítulo 4.

Para a implementação do algoritmo de Newton-Raphson, é necessária a matriz de informação

$$
\mathbf{F}_{n}(\boldsymbol{\theta})=-\frac{\partial^{2} \log L_{n}(\boldsymbol{\theta})}{\partial \boldsymbol{\theta} \partial \boldsymbol{\theta}^{\prime}}=-\left(\begin{array}{ccc}
\frac{\partial^{2} \log L_{n}(\boldsymbol{\theta})}{\partial \boldsymbol{\beta} \partial \boldsymbol{\beta}^{\prime}} & \frac{\partial^{2} \log L_{n}(\boldsymbol{\theta})}{\partial \boldsymbol{\beta} \partial \boldsymbol{\alpha}^{\prime}} & \frac{\partial^{2} \log L_{n}(\boldsymbol{\theta})}{\partial \boldsymbol{\beta} \partial \delta} \\
\frac{\partial^{2} \log L_{n}(\boldsymbol{\theta})}{\partial \boldsymbol{\alpha} \partial \boldsymbol{\beta}^{\prime}} & \frac{\partial^{2} \log L_{n}(\boldsymbol{\theta})}{\partial \boldsymbol{\alpha} \partial \boldsymbol{\alpha}^{\prime}} & \frac{\partial^{2} \log L_{n}(\boldsymbol{\theta})}{\partial \boldsymbol{\alpha} \partial \delta} \\
\frac{\partial^{2} \log L_{n}(\boldsymbol{\theta})}{\partial \delta \partial \boldsymbol{\beta}^{\prime}} & \frac{\partial^{2} \log L_{n}(\boldsymbol{\theta})}{\partial \delta \partial \boldsymbol{\alpha}^{\prime}} & \frac{\partial^{2} \log L_{n}(\boldsymbol{\theta})}{\partial \delta^{2}}
\end{array}\right)_{r \times r}=\sum_{i=1}^{n} \mathbf{Z}_{\mathbf{i}} \mathcal{F}_{i}(\boldsymbol{\theta}) \mathbf{Z}_{\mathbf{i}}{ }^{\prime}
$$

em que $\mathcal{F}_{i}(\boldsymbol{\theta})$ é uma matriz simétrica de dimensão $3 \times 3$, cujas componentes $f_{i}^{j k}$, $j, k=1,2,3$ são detalhadas no Apêndice A. 
A partir de um valor inicial para o parâmetro $\boldsymbol{\theta}$, o processo iterativo tem como núcleo

$$
\boldsymbol{\theta}_{K+1}=\boldsymbol{\theta}_{K}+\mathbf{F}_{n}^{-1}\left(\boldsymbol{\theta}_{K}\right) \mathbf{U}_{n}\left(\boldsymbol{\theta}_{K}\right),
$$

em que $\boldsymbol{\theta}_{K}$ e $\boldsymbol{\theta}_{K+1}$ são as estimativas de $\boldsymbol{\theta}$ nos passos $K$ e $K+1$, respectivamente, e $\mathbf{F}_{n}^{-1}$ é a inversa da matriz $\mathbf{F}_{n}$.

A expressão (2.3) é utilizada recursivamente até que $\left\|\boldsymbol{\theta}_{K+1}-\boldsymbol{\theta}_{K}\right\|<\epsilon$, com $\epsilon$ igual a um valor pré especificado. Ao atingir a convergência obtem-se $\hat{\boldsymbol{\theta}}$, o e.m.v. de $\boldsymbol{\theta}$, que satisfaz

$$
\mathbf{U}_{n}(\hat{\boldsymbol{\theta}})=\mathbf{0}
$$

A matriz $\mathbf{F}_{n}^{-1}(\hat{\boldsymbol{\theta}})$ fornece um estimador consistente para a matriz de covariância de $\hat{\boldsymbol{\theta}}$, sob algumas condições (ver, por exemplo, Teoremas 8.2 e 8.3, (Maller \& Zhou 1996)).

Segundo Maller \& Zhou (1996), existem alguns problemas no processo iterativo de Newton-Raphson. Primeiro, assume-se que a matriz de segundas derivadas tem inversa em cada valor de $\boldsymbol{\theta}_{K}$ e em um ponto estacionário $\hat{\boldsymbol{\theta}}$. Se $\mathbf{F}_{n}(\boldsymbol{\theta})$ for singular no ponto $\boldsymbol{\theta}$, a superfície da verossimilhança não é côncava próximo ao ponto estacionário, o que pode trazer dificuldades. Outra limitação é que, como a variação do parâmetro $\pi_{i}$ é restrita ao intervalo $(0,1]$, a verossimilhança pode ser maximizada na borda do espaço paramétrico. Esta não é uma situação prática incomum, pois usualmente ocorreria quando os dados apresentassem uma pequena (ou nenhuma) proporção de curados, levando a se descartar o uso de modelos com fração de cura.

\subsection{Modelo semiparamétrico com fração de cura}

O modelo de Cox com fração de cura, proposto originalmente por Kuk \& Chen (1992), assume que o efeito das covariáveis sobre os tempos de falha de pacientes não curados 
é especificado por um modelo de Cox. Inicialmente, define-se a variável binária $b$ como indicadora de "não cura", isto é, $b_{i}=1$ se o $i$-ésimo paciente é não curado e $b_{i}=0$ se o $i$-ésimo paciente é curado.

Dado um vetor de covariáveis $\mathbf{Z}_{1}$ de dimensão $r_{1} \times 1$, a função de risco condicional a pacientes não curados é dada por

$$
h_{N C}(t)=h\left(t \mid b=1 ; \mathbf{Z}_{1}\right)=h_{0}\left(t \mid b=1 ; \mathbf{Z}_{1}\right) \mathrm{e}^{\mathbf{Z}_{1}^{\prime} \boldsymbol{\beta}}=h_{N C}^{0}(t) \mathrm{e}^{\mathbf{Z}_{1}^{\prime} \boldsymbol{\beta}}, \quad \boldsymbol{\beta} \in \mathbb{R}^{r_{1}}
$$

em que $h_{N C}^{0}($.$) é a função de risco padrão condicional e a função de sobrevivência$ condicional é

$$
S_{N C}(t)=S\left(t \mid b=1 ; \mathbf{Z}_{1}\right)=\left[S_{0}(t \mid b=1)\right]^{\mathrm{e}^{\left(\mathbf{Z}_{1}^{\prime} \boldsymbol{\beta}\right)}}=\left[S_{N C}^{0}(t)\right]^{\mathrm{e}^{\mathbf{z}_{1}{ }^{\prime} \boldsymbol{\beta}}}
$$

em que $S_{N C}^{0}(t)$ é a função de sobrevivência padrão condicional a pacientes não curados e $\boldsymbol{\beta}$ é o vetor de parâmetros desconhecidos associados ao vetor de covariáveis $\mathbf{Z}_{1}$.

Assim como no modelo paramétrico, a probabilidade de não cura $\pi_{i}$ associada ao indivíduo $i$ é especificada por um modelo logístico com vetor $r_{2}$ x 1 de covariáveis $\mathbf{Z}_{2 i}$ e vetor de parâmetros $\boldsymbol{\alpha} \in \mathbb{R}^{r_{2}+1}$. Portanto, os parâmetros a serem estimados no modelo semiparamétrico com fração de cura são $\boldsymbol{\alpha}, \boldsymbol{\beta}$ e $S_{N C}^{0}(t)$. A estimação de $\boldsymbol{\alpha}$ e $\boldsymbol{\beta}$ é baseada nos modelos usuais de regressão logística e de Cox. Já a implementação computacional da estimação de $S_{N C}^{0}(t)$ requer mais cuidado pois, como este parâmetro tem dimensão infinita, existem algumas dificuldades que serão discutidas adiante.

Kuk \& Chen (1992) propõem estimar os parâmetros $\boldsymbol{\alpha}$ e $\boldsymbol{\beta}$ por um método de simulação de Monte Carlo a fim de obter uma aproximação da função de verossimilhança. Este método requer programas específicos para implementar a simulação, o que o torna menos atrativo sob o ponto de vista prático. A utilização do algoritmo EM para estimação desses parâmetros foi proposta inicialmente por Taylor (1995) para modelos 
mais simples e posteriormente estendida por Sy \& Taylor (2000) e Peng \& Dear (2000). Com o objetivo de facilitar a implementação destes métodos, Peng (2003b) sugere uma nova aproximação que permite o uso de funções disponíveis em programas estatísticos convencionais conforme descrito a seguir.

Note que se o indivíduo $i$ "falha" $\left(\Delta_{i}=1\right)$, necessariamente ele é não curado $\left(b_{i}=1\right)$. No entanto, se o indivíduo é censurado $\left(\Delta_{i}=0\right)$, ele pode ser curado ou não $\left(b_{i}=0\right.$ ou $\left.b_{i}=1\right)$. Logo, ao se observar os indicadores de falha, os valores de $b_{i}$ são parcialmente observados. Peng (2003b) sugere escrever a verossimilhança completa, supondo que $\mathbf{b}=\left(b_{i}, i=1, \ldots, n\right)$ é observável. Conforme (2.1), em modelos com fração de cura, se a $i$-ésima observação é uma falha, sua contribuição para a verossimilhança é $\pi_{i} f_{N C}\left(t_{i}\right)$, que neste caso é igual a $\left[\pi f_{N C}\left(t_{i}\right)\right]^{b_{i}}$, pois para as falhas, $b_{i}=1, \forall i$. Quando o indivíduo $i$ é censurado, a contribuição é dada pela função de sobrevivência $S\left(t_{i}\right)=P\left(T>t_{i}\right)$ que, no caso de dados com proporção de curados, é

$$
S\left(t_{i}\right)=\left\{\begin{array}{c}
1-\pi_{i}, \quad \text { se } b_{i}=0(\text { curado }) \\
\pi_{i} S_{N C}\left(t_{i}\right) \quad \text { se } b_{i}=1(\text { não curado })
\end{array}\right.
$$

Logo, é possível reescrever a função de verossimilhança dada em (2.1) incluindo a informação sobre a cura $\left(b_{i}\right)$, isto é,

$$
\begin{aligned}
L_{n}(\boldsymbol{\theta}) & =\prod_{i=1}^{n}\left[\left(\pi_{i} f_{N C}\left(t_{i}\right)\right)^{b_{i}}\right]^{\Delta_{i}}\left[\left(1-\pi_{i}\right)^{\left(1-b_{i}\right)}\left(\pi_{i} S_{N C}\left(t_{i}\right)\right)^{b_{i}}\right]^{1-\Delta_{i}} \\
& =\prod_{i=1}^{n}\left(\frac{\pi_{i}^{b_{i}} f_{N C}\left(t_{i}\right)^{b_{i}}}{\pi_{i}^{b_{i}}\left(S_{N C}\left(t_{i}\right)\right)^{b_{i}}} \frac{1}{\left(1-\pi_{i}\right)^{\left(1-b_{i}\right)}}\right)^{\Delta_{i}}\left(1-\pi_{i}\right)^{\left(1-b_{i}\right)}\left(\pi_{i} S_{N C}\left(t_{i}\right)\right)^{b_{i}} \\
& =\prod_{i=1}^{n} \frac{\left(f_{N C}\left(t_{i}\right) / S_{N C}\left(t_{i}\right)\right)^{b_{i} \Delta_{i}}}{\left(1-\pi_{i}\right)^{\left(1-b_{i}\right) \Delta_{i}}}\left(1-\pi_{i}\right)^{\left(1-b_{i}\right)}\left(\pi_{i}\right)^{b_{i}}\left(S_{N C}\left(t_{i}\right)\right)^{b_{i}}
\end{aligned}
$$

Usando o fato que $h(t)=f(t) / S(t), b_{i} \Delta_{i}=\Delta_{i}$ e $\left(1-b_{i}\right) \Delta_{i}=0$, a função $L_{n}(\boldsymbol{\theta})$ 
pode ser escrita como

$$
\begin{aligned}
L_{n}(\boldsymbol{\theta}) & =\prod_{i=1}^{n} \pi_{i}^{b_{i}}\left(1-\pi_{i}\right)^{\left(1-b_{i}\right)} \prod_{i=1}^{n}\left[h_{N C}\left(t_{i}\right)\right]^{\Delta_{i}}\left[S_{N C}\left(t_{i}\right)\right]^{b_{i}} \\
& =\prod_{i=1}^{n} \pi_{i}^{b_{i}}\left(1-\pi_{i}\right)^{\left(1-b_{i}\right)} \prod_{i=1}^{n}\left[h_{N C}^{0}\left(t_{i}\right) \mathrm{e}^{\mathbf{Z}_{1 i}^{\prime} \boldsymbol{\beta}}\right]^{\Delta_{i}}\left[S_{N C}^{0}\left(t_{i}\right)\right]^{b_{i} \mathrm{e}^{\mathbf{Z}_{1 i}^{\prime} \boldsymbol{\beta}}} \\
& =L_{1}(\boldsymbol{\alpha} ; \mathbf{b}) L_{2}\left(\boldsymbol{\beta} ; \mathbf{S}_{N C}^{0} ; \mathbf{b}\right)
\end{aligned}
$$

em que $\mathbf{b}=\left(b_{1}, \ldots, b_{n}\right)$ é o vetor de indicadores de não cura, $\mathbf{S}_{N C}^{0}=\left(S_{N C}^{0}\left(t_{1}\right), \ldots, S_{N C}^{0}\left(t_{n}\right)\right)$ é a função de sobrevivência padrão calculada nos tempos de falha ou censura observados, $\boldsymbol{\beta}$ é o vetor de parâmetros relacionado ao termo de sobrevivência (modelo de Cox) e $\boldsymbol{\alpha}$ é o vetor de parâmetros correspondente ao modelo logístico para a probabilidade de não cura $\pi_{i}$, sendo que $\pi_{i}$ está associado a um vetor de covariáveis $\mathbf{Z}_{2 i}$, da mesma forma que no modelo paramétrico.

Note que $L_{1}$ corresponde à função de verossimilhança de um modelo logístico usual no qual $b_{i}$ tem distribuição de Bernoulli com parâmetro $\pi_{i}$. Pode-se notar também que o termo $L_{2}$ corresponde à função de verossimilhança de um modelo de sobrevivência usual incluindo a covariável $\log b_{i}$ com coeficiente constante igual a 1 conforme verificase a seguir.

$$
\begin{aligned}
L_{2}\left(\boldsymbol{\beta} ; \mathbf{S}_{N C}^{0} ; \mathbf{b}\right) & =\prod_{i=1}^{n}\left(h_{N C}\left(t_{i}\right)\right)^{\Delta_{i}}\left(S_{N C}\left(t_{i}\right)\right)^{b_{i}}=\prod_{i=1}^{n}\left(\frac{f_{N C}\left(t_{i}\right)}{S_{N C}\left(t_{i}\right)}\right)^{\Delta_{i}}\left(S_{N C}\left(t_{i}\right)\right)^{b_{i}} \\
& =\prod_{i=1}^{n}\left(f_{N C}\left(t_{i}\right)\right)^{\Delta_{i}}\left(S_{N C}\left(t_{i}\right)\right)^{b_{i}-\Delta_{i}}=\prod_{i=1}^{n}\left(f_{N C}\left(t_{i}\right)\right)^{\Delta_{i}}\left(S_{N C}\left(t_{i}\right)^{b_{i}}\right)^{1-\Delta_{i}} \\
& =\prod_{i=1}^{n}\left(f_{N C}\left(t_{i}\right)\right)^{\Delta_{i}}\left(S_{N C}^{0}\left(t_{i}\right)^{b_{i} \mathrm{e}^{\prime}{ }_{1 i}^{\beta}}\right)^{1-\Delta_{i}} \\
& =\prod_{i=1}^{n}\left(f_{N C}\left(t_{i}\right)\right)^{\Delta_{i}}\left(S_{N C}^{0}\left(t_{i}\right)^{\mathrm{e}^{\log b_{i}+\mathbf{z}_{1 i}^{\prime} \boldsymbol{\beta}}}\right)^{1-\Delta_{i}}
\end{aligned}
$$


Portanto, como a função de verossimilhança pode ser escrita como produto de funções de verossimilhança conhecidas, uma Bernoulli envolvendo os parâmetros associados à probabilidade de cura e outra com os parâmetros do modelo de Cox, não haveria problemas no processo de estimação caso os valores de $b_{i}$ fossem completamente observáveis.

\subsubsection{Estimação dos parâmetros - Algoritmo EM}

A proposta de Peng (2003b) é utilizar o algoritmo EM, substituindo $b_{i}$ por uma estimativa de sua esperança condicional, dadas as estimativas dos parâmetros em cada iteração. Segundo o autor, a vantagem de usar o algoritmo EM é que como os parâmetros $\boldsymbol{\alpha}, \boldsymbol{\beta}$ e $\mathbf{S}_{N C}^{0}$ podem ser estimados separadamente, pode-se utilizar programas estatísticos usuais para regressão logística e regressão de Cox, desde que permitam a inclusão de covariáveis com coeficientes fixos (offset) e forneçam estimativas para a função de sobrevivência padrão.

Seja $p_{i}^{(k)}$ a esperança estimada de $b_{i}$, condicional às estimativas de $\boldsymbol{\alpha}, \boldsymbol{\beta}$ e $S_{N C}^{0}\left(t_{i}\right)$ obtidas na iteração $k$, isto é, $p_{i}^{(k)}=E\left(b_{i} \mid \boldsymbol{\alpha}^{(k)}, \boldsymbol{\beta}^{(k)}, S_{N C}^{0(k)}\left(t_{i}\right)\right), i=1, \ldots, n$.

O algoritmo EM consiste em aplicar iterativamente os seguintes passos:

Passo E: A partir das estimativas de $\boldsymbol{\alpha}^{(k)}, \boldsymbol{\beta}^{(k)}$ e $S_{N C}^{0(k)}\left(t_{i}\right)$ obtidas na k-ésima iteração, atualizar a estimativa da esperança condicional $p_{i}$.

Passo M: Com a estimativa de $\mathbf{p}=\left(p_{1}, \ldots, p_{n}\right)$ gerada no passo E, maximizar as funções de verossimilhança $L_{1}(\boldsymbol{\alpha} ; \mathbf{p})$ e $L_{2}\left(\boldsymbol{\beta} ; \mathbf{S}_{N C}^{0} ; \mathbf{p}\right)$, usando um método iterativo como Newton-Raphson ou Scoring de Fisher para obter uma atualização de $\boldsymbol{\alpha}, \boldsymbol{\beta}$ e $\mathbf{S}_{N C}^{0}$.

No passo M, o parâmetro $\boldsymbol{\alpha}$ é estimado através da maximização de $L_{1}(\boldsymbol{\alpha} ; \mathbf{p})$ e os 
parâmetros $\boldsymbol{\beta}$ e $\mathbf{S}_{N C}^{0}$ em $L_{2}$ são estimados da forma usual do modelo de Cox, isto é, estima-se $\boldsymbol{\beta}$ por máxima verossimilhança parcial e $\mathbf{S}_{N C}^{0}$ é estimada na seqüência pela fórmula de Breslow modificada apresentada em (2.10).

Os passos são aplicados repetidamente até que se atinja algum critério de convergência pré-estabelecido. Os detalhes de cada passo são apresentados a seguir.

\section{Atualização de $p_{i}$ no passo E}

Para a atualização de $p_{i}$ considere o seguinte esquema: a esperança de $b_{i}$, condicional aos valores de $\boldsymbol{\alpha}, \boldsymbol{\beta}$ e $S_{N C}^{0}\left(t_{i}\right)$, é dada por

$$
p_{i}=E\left(b_{i} \mid \boldsymbol{\alpha}, \boldsymbol{\beta}, S_{N C}^{0}\left(t_{i}\right)\right)=P\left(b_{i}=1 \mid \boldsymbol{\alpha}, \boldsymbol{\beta}, S_{N C}^{0}\left(t_{i}\right)\right)
$$

Como existe uma relação entre a cura $\left(b_{i}\right)$ e o evento $\left(\Delta_{i}\right)$, a probabilidade de cura depende de a observação ser falha ou censura. Note que

$$
\begin{aligned}
P\left(b_{i}=1 \mid \Delta_{i}=1\right) & =\frac{P\left(\Delta_{i}=1 \mid b_{i}=1\right) P\left(b_{i}=1\right)}{P\left(\Delta_{i}=1 \mid b_{i}=0\right) P\left(b_{i}=0\right)+P\left(\Delta_{i}=1 \mid b_{i}=1\right) P\left(b_{i}=1\right)} \\
& =\frac{P\left(\Delta_{i}=1 \mid b_{i}=1\right) \pi_{i}}{0 .\left(1-\pi_{i}\right)+P\left(\Delta_{i}=1 \mid b_{i}=1\right) \pi_{i}}=1, \\
P\left(b_{i}=1 \mid \Delta_{i}=0\right) & =\frac{P\left(\Delta_{i}=0 \mid b_{i}=1\right) P\left(b_{i}=1\right)}{P\left(\Delta_{i}=0 \mid b_{i}=0\right) P\left(b_{i}=0\right)+P\left(\Delta_{i}=0 \mid b_{i}=1\right) P\left(b_{i}=1\right)} \\
& =\frac{P\left(T>t_{i} \mid b_{i}=1\right) \pi_{i}}{P\left(T>t_{i} \mid b_{i}=0\right)\left(1-\pi_{i}\right)+P\left(T>t_{i} \mid b_{i}=1\right) \pi_{i}} \\
& =\frac{S_{N C}\left(t_{i}\right) \pi_{i}}{\left(1-\pi_{i}\right)+S_{N C}\left(t_{i}\right) \pi_{i}} .
\end{aligned}
$$


Logo, para os indivíduos que "falham", $P\left(b_{i}=1 \mid \boldsymbol{\alpha}, \boldsymbol{\beta}, S_{N C}^{0}\left(t_{i}\right), \Delta_{i}=1\right)=1$, pois dado que o paciente "falhou", a única possibilidade é que ele seja não curado. Para os indivíduos censurados, $P\left(b_{i}=1 \mid \boldsymbol{\alpha}, \boldsymbol{\beta}, S_{N C}^{0\left(t_{i}\right)}, \Delta_{i}=0\right)=\pi_{i} S_{N C}\left(t_{i}\right) / S\left(t_{i}\right)$, pois $S(t)=1-\pi+\pi S_{N C}(t)$.

Portanto, dados $\boldsymbol{\alpha}^{(k)}, \boldsymbol{\beta}^{(k)}$ e $S_{N C}^{0(k)}\left(t_{i}\right)$, as estimativas obtidas na iteração $k$, a estimativa de $p_{i}$ nesta iteração é:

$$
\begin{aligned}
p_{i}^{(k)} & =E\left(b_{i} \mid \boldsymbol{\alpha}^{(k)}, \boldsymbol{\beta}^{(k)}, S_{N C}^{0(k)}\left(t_{i}\right)\right) \\
& =\Delta_{i}+\left(1-\Delta_{i}\right) \frac{\pi_{i}^{(k)} S_{N C}^{0(k)}\left(t_{i}\right)^{\mathrm{e}^{\left(\mathbf{Z}_{1 i}^{\prime} \boldsymbol{\beta}^{(k)}\right)}}}{1-\pi_{i}^{(k)}+\pi_{i}^{(k)} S_{N C}^{0(k)}\left(t_{i}\right)^{\mathrm{e}^{\left(\mathbf{Z}_{1 i}^{\prime} \boldsymbol{\beta}^{(k)}\right)}}}
\end{aligned}
$$

em que $\pi_{i}^{(k)}=\frac{\mathrm{e}^{\left(Z_{2 i}^{\prime} \alpha^{(k)}\right)}}{1+\mathrm{e}^{\left(Z_{2 i}^{\prime}{ }^{(k)}\right)}}$

No processo de estimação sugerido, a cada iteração é preciso obter as estimativas da função de sobrevivência padrão condicionalmente a pacientes não $\operatorname{curados}\left(S_{N C}^{0}\left(t_{i}\right), i=\right.$ $1, \ldots, n)$. No caso de modelos com fração de cura, $S_{N C}^{0}(t)$ contém informação sobre os parâmetros de interesse e também precisa ser estimada.

A função de sobrevivência padrão do modelo com fração de cura pode ser estimada utilizando-se métodos semelhantes aos usados no modelo de Cox. O programa computacional de Peng (2003b) utiliza uma adaptação do estimador de Breslow [Peng \& Dear (2000)], porém outros métodos podem ser considerados, como o estimador produto limite [Sy \& Taylor (2000)].

\section{Maximização de $L_{1}$ e $L_{2}$ - passo $\mathbf{M}$}

O passo E do algoritmo EM consiste basicamente em atribuir probabilidades de não cura para cada indivíduo. O passo M consiste em maximizar a função de verossimi- 
lhança, substituindo o indicador de não cura $b_{i}$ por uma estimativa de sua esperança condicional $\left(p_{i}\right)$, a fim de obter novas estimativas para $\boldsymbol{\alpha}, \boldsymbol{\beta}$ e $S_{N C}^{0}\left(t_{i}\right)$.

Tomando o logaritmo da verossimilhança (2.4), tem-se

$$
\log L_{n}(\boldsymbol{\theta})=\log L_{1}(\boldsymbol{\alpha} ; \mathbf{b})+\log L_{2}\left(\boldsymbol{\beta} ; \mathbf{S}_{N C}^{0} ; \mathbf{b}\right)
$$

Supondo b conhecido, as estimativas de máxima verossimilhança de $\boldsymbol{\alpha}$ podem ser obtidas separadamente das estimativas de $\boldsymbol{\beta}$ e $\mathbf{S}_{N C}^{0}$ pois, neste caso, o primeiro termo da soma em (2.6) só depende de $\boldsymbol{\alpha}$ e o segundo termo só depende de $\boldsymbol{\beta}$ e $\mathbf{S}_{N C}^{0}$. Este fato facilita consideravelmente o processo de estimação.

Logo, a partir do valor de $p_{i}^{(k)}$ calculado por (2.5) no passo E, obtem-se $\boldsymbol{\alpha}^{(k+1)}$ maximizando a função

$$
\log L_{1}\left(\boldsymbol{\alpha} ; p_{i}^{(k)}\right)=\sum_{i=1}^{n} p_{i}^{(k)} \log \pi_{i}(\boldsymbol{\alpha})+\left(1-p_{i}^{(k)}\right) \log \left(1-\pi_{i}(\boldsymbol{\alpha})\right)
$$

que corresponde ao logaritmo da verossimilhanca de um modelo logístico usual.

Métodos convencionais também podem ser utilizados para estimar $\boldsymbol{\beta}$ e $\mathbf{S}_{N C}^{0}$. Como a verossimilhança $L_{2}$ corresponde a uma função de verossimilhança para dados de sobrevivência sob o modelo de Cox, sua maximização na verdade é feita pelo método usual de verossimilhança parcial, o que implica que não é necessário conhecer $\mathbf{S}_{N C}^{0}$ para estimar o parâmetro $\boldsymbol{\beta}$. A partir da estimativa de $\boldsymbol{\beta}$, obtem-se $\hat{\mathbf{S}}_{N C}^{0}$ por meio de um estimador de Breslow modificado descrito a seguir. 
Estimação de $S_{N C}^{0}(t)$

O método descrito aqui é adotado por Peng (2003b) e é baseado na abordagem proposta por Kalbfleisch \& Prentice (2002). A idéia básica é escrever a verossimilhança em termos da função de sobrevivência e obter $\hat{S}_{N C}^{0}(t)$ por meio de uma verossimilhança perfilada, assumindo que $\boldsymbol{\beta}$ tem o mesmo valor que seu estimador de máxima verossimilhança $\hat{\boldsymbol{\beta}}$.

Sejam $\tau_{1}<\tau_{2}<\ldots<\tau_{L}$ os tempos distintos de falha. Por convenção, define-se que $\tau_{0}=0$ e $\tau_{L+1}=\infty$. Seja $D_{j}$ o conjunto de indivíduos que falham em $\tau_{j}$ e $R_{j}$ o conjunto de indivíduos em risco no instante $\tau_{j}$, isto é, que não falharam nem foram censurados até o instante imediatamente anterior a $\tau_{j}$.

Seguindo o argumento de Kalbfleisch \& Prentice (2002), a contribuição para a verossimilhança de um paciente que falha em $\tau_{j}$ é $\left[S_{N C}^{0}\left(\tau_{j}\right)\right]^{\mathrm{e}^{\mathbf{z}_{1 i}^{\prime} \boldsymbol{\beta}}}-\left[S_{N C}^{0}\left(\tau_{j}+0\right)\right]^{\mathrm{e}_{1 i}^{\prime} \boldsymbol{\beta}} \mathrm{e}$ a contribuição de um paciente que é censurado em $\tau_{j}$ é $\left[S_{N C}^{0}\left(\tau_{j}+0\right)\right]^{\mathrm{e}_{1 i}^{\prime} \boldsymbol{\beta}}$.

Como $S_{N C}^{0}(t)$ é uma função de sobrevivência, ela é não crescente e contínua à esquerda e então deve ser constante exceto nos tempos de falha. Assume-se que $S_{N C}^{0}(t+$ $0)=S_{N C}^{0}\left(\tau_{j}+0\right)$ para $t \in\left[\tau_{j}, \tau_{j+1}\right)$, o que leva a uma distribuição discreta com massa apenas nas observações não censuradas.

$$
\begin{aligned}
& \text { Tomando } g_{j}=S_{N C}^{0}\left(\tau_{j+1}\right) / S_{N C}^{0}\left(\tau_{j}\right), j=1, \ldots, L, \text { tem-se que } \\
& g_{0}=1, \quad S_{N C}^{0}(t)=\prod_{j: \tau_{j}<t} g_{j}, \quad S_{N C}^{0}\left(\tau_{j}\right)=\prod_{i=1}^{j-1} g_{i} \quad \text { e } S_{N C}^{0}\left(\tau_{j}+0\right)=\prod_{i=1}^{j} g_{i} .
\end{aligned}
$$

Portanto, substituindo $b_{i}$ por sua esperança condicional $p_{i}$, a função de verossimilhança $L_{2}$ pode ser reescrita como 


$$
\prod_{j=1}^{L}\left[\prod_{i \in D_{j}}\left(1-g_{j}^{\mathrm{e}^{\mathbf{z}_{1}^{\prime} \beta}}\right) \prod_{i \in R_{j}-D_{j}} g_{j}^{p_{i}} \mathrm{e}^{\mathbf{z}_{1 i}^{\prime} \beta}\right]
$$

A estimativa da função de sobrevivência padrão é obtida maximizando (2.8) com respeito a $g_{j}$, dadas as estimativas de $\boldsymbol{\beta}$. O logaritmo da função em (2.8) é dado por

$$
\log L_{2}=\sum_{j=1}^{L}\left\{\sum_{i \in D_{j}} \log \left(1-g_{j}^{\mathrm{e}^{\mathbf{Z}_{1 i}^{\prime} \boldsymbol{\beta}}}\right)+\sum_{i \in R_{j}-D_{j}} p_{i} \mathrm{e}^{\mathbf{Z}_{1 i}^{\prime} \boldsymbol{\beta}} \log g_{j}\right\}
$$

Derivando em relação a $g_{j}$,

$$
\begin{aligned}
\frac{\partial \log L_{2}}{\partial g_{j}} & =\sum_{i \in D_{j}}-\frac{\mathrm{e}^{\mathbf{Z}_{1 i}^{\prime} \boldsymbol{\beta}} g_{j}^{\mathrm{e}^{\mathbf{Z}_{1 i} \boldsymbol{\beta}}-1}}{\left(1-g_{j}^{\mathrm{e}_{1 i}^{\prime} \boldsymbol{\beta}}\right)}+\sum_{i \in R_{j}-D_{j}} \frac{p_{i} \mathrm{e}^{\mathbf{Z}_{1 i}^{\prime} \boldsymbol{\beta}}}{g_{j}} \\
& =\frac{1}{g_{j}} \sum_{i \in R_{j}} p_{i} \mathrm{e}^{\mathbf{Z}_{1 i}^{\prime} \boldsymbol{\beta}}-\frac{1}{g_{j}} \sum_{i \in D_{j}} \frac{\mathrm{e}^{\mathbf{Z}_{1 i}^{\prime} \boldsymbol{\beta}}}{\left(1-g_{j}^{\mathrm{e}^{\mathbf{Z}_{1 i}^{\prime} \boldsymbol{\beta}}}\right)}, \quad j=1, \ldots, L .
\end{aligned}
$$

A equação de estimação obtida ao se igualar a derivada acima a zero é

$$
\sum_{i \in R_{j}} p_{i} \mathrm{e}^{\mathbf{Z}_{1 i}^{\prime} \boldsymbol{\beta}}=\sum_{i \in D_{j}} \frac{\mathrm{e}^{\mathbf{Z}_{1 i}^{\prime} \boldsymbol{\beta}}}{\left(1-\hat{g}_{j}^{\mathrm{e}^{\mathbf{Z}_{i i}^{\prime} \boldsymbol{\beta}}}\right)} \quad j=1, \ldots, L
$$

que não tem solução analítica; o estimador de máxima verossimilhança de $g_{j}$ pode ser obtido iterativamente utilizando o método de Newton-Raphson.

O processo iterativo pode ser evitado se for utilizada uma aproximação para a soma do membro direito de (2.9). O termo $\hat{g}_{j}^{\mathrm{e}_{1 i}^{\prime} \beta}$ pode ser escrito como

$$
\exp \left\{\mathrm{e}^{\mathbf{Z}_{1 i}^{\prime} \boldsymbol{\beta}} \log \hat{g}_{j}\right\}
$$


e tomando os dois primeiros termos da expansão da exponencial ( $\left.\mathrm{e}^{x}=\sum_{k=0}^{\infty} \frac{x^{k}}{k !}\right)$, tem-se a aproximação

$$
\exp \left\{\mathrm{e}^{\mathbf{Z}_{1 i}^{\prime} \boldsymbol{\beta}} \log \hat{g}_{j}\right\} \approx 1+\mathrm{e}^{\mathbf{Z}_{1 i}^{\prime} \boldsymbol{\beta}} \log \hat{g}_{j}
$$

Substituindo $\hat{g}_{j}^{\mathrm{e}^{\prime}{ }^{\prime} \boldsymbol{\beta}}$ em (2.9) pela aproximação acima, tem-se que

$$
\sum_{i \in R_{j}} p_{i} \mathrm{e}^{\mathbf{Z}_{1 i}^{\prime} \boldsymbol{\beta}} \approx \sum_{i \in D_{j}} \frac{\mathrm{e}^{\mathbf{Z}_{1 i}^{\prime} \boldsymbol{\beta}}}{\left(1-1-\mathrm{e}^{\mathbf{Z}_{1 i}^{\prime} \boldsymbol{\beta}} \log \hat{g}_{j}\right)} \Longrightarrow-\sum_{i \in R_{j}} p_{i} \mathrm{e}^{\mathbf{Z}_{1 i}^{\prime} \boldsymbol{\beta}} \approx \frac{d_{j}}{\log \hat{g}_{j}}
$$

em que $d_{j}$ é o número de falhas em $\tau_{j}$.

Portanto, supondo $p_{i}$ e $\boldsymbol{\beta}$ conhecidos, o estimador de máxima verossimilhança de $g_{j}$ pode ser aproximado por

$$
\hat{g_{j}} \approx \exp \left\{\frac{-d_{j}}{\sum_{i \in R_{j}} p_{i} \mathrm{e}^{\prime}{ }_{1 i}^{\boldsymbol{\beta}}}\right\}
$$

Como $S_{N C}^{0}(t)=\prod_{j: \tau_{j}<t} g_{j}$, a função de sobrevivência padrão, supondo $p_{i}$ conhecido, pode ser estimada por

$$
\hat{S}_{N C}^{0}(t)=\exp \left\{-\sum_{j: \tau_{j}<t} \frac{d_{j}}{\sum_{i \in R_{j}} p_{i} \mathrm{e}^{\mathbf{Z}_{1 i}^{\prime} \hat{\boldsymbol{\beta}}}}\right\}
$$

em que $\hat{\boldsymbol{\beta}}$ é o estimador de máxima verossimilhança parcial de $\boldsymbol{\beta}$.

Este estimador corresponde a uma generalização da fórmula de Breslow para modelos com fração de cura e inclui $p_{i}$, a esperança condicional de $b_{i}$, no denominador. 
Isto implica em considerar o modelo de Cox usual com a inclusão de uma covariável $\log p_{i}$ com coeficiente fixo igual a 1 para a estimação de $\boldsymbol{\beta}$.

No processo de estimação do algoritmo $\operatorname{EM}, S_{N C}^{0(k+1)}\left(t_{i}\right)$ é atualizado por $(2.10)$ substituindo $\hat{\boldsymbol{\beta}}$ e $p_{i}$ pelos valores de $\boldsymbol{\beta}^{(k+1)}$ e $p_{i}^{(k)}$, respectivamente. Note que a cada iteração, as estimativas de $S_{N C}^{0}\left(t_{i}\right)$ devem ser calculadas em todos os tempos individuais observados, o que torna o processo de estimação bastante demorado.

Um fator importante a ser considerado é que as estimativas da função de sobrevivência obtidas a partir de (2.10) não se aproximam de zero quando $t \rightarrow \infty$, a menos que não existam observações censuradas com tempos maiores que o maior tempo de falha $\tau_{L}$. Em dados com proporções de curados, observações censuradas com tempos de sobrevivência maiores que o maior tempo de falha são comuns pois elas evidenciam a presença de curados na amostra. Isto implica uma distribuição imprópria para pacientes não curados e, como conseqüência, gera um problema de identificabilidade (Peng 2003a).

Uma alternativa é fixar ou completar a cauda da distribuição de $S_{N C}(t)$. Taylor (1995) sugere impor que as estimativas sejam nulas nos tempos maiores que o maior tempo de falha, isto é, $\hat{S}_{N C}^{0}(t)=0, \forall t>\tau_{L}$. Isto implica supor que todos os indivíduos com tempos de sobrevivência maiores que $\tau_{L}$ são curados. Peng (2003a) propõe usar uma distribuição exponencial ou Weibull para estimar a cauda de modo que, a partir de $\tau_{L}, \hat{S}_{N C}(t)$ descresça suavemente até zero.

Outros métodos podem ser considerados para estimar a função de sobrevivência padrão em modelos com fração de cura, como o estimador produto limite (Sy \& Taylor 2000). Na aproximação de Monte Carlo para a verossimilhança marginal sugerida por Kuk \& Chen (1992), a função padrão é eliminada. Com os parâmetros de regressão fixados nos valores das estimativas, eles maximizam a verossimilhança completa via algoritmo EM para estimar a função de sobrevivência padrão. 


\section{Precisão das estimativas}

Nos métodos de estimação sugeridos por Peng \& Dear (2000) e Sy \& Taylor (2000), os erros padrão não podem ser obtidos diretamente a partir das iterações do algoritmo EM. O uso da inversa da matriz de informação também não é simples, uma vez que a dimensão da matriz pode ser muito grande, principalmente quando houver muitos tempos distintos de falha devido à estimação de $S_{N C}^{0}$. Segundo Peng (2003b), deve-se levar em conta a instabilidade numérica da inversão de uma matriz muito grande e a adequabilidade deste procedimento no processo de estimação. O autor propõe utilizar a técnica bootstrap (Davison \& Hinkley 1997) para calcular a variância dos parâmetros estimados. Apesar de ser um método computacionalmente intensivo, a idéia é simples e de fácil implementação.

\section{Implementação do algoritmo EM}

Os passos para implementação do algoritmo EM detalhados anteriormente são resumidos a seguir.

- 1. Inicializar os valores de $p_{i}$. Como $p_{i}$ é a probabilidade de o paciente $i$ ser não curado, uma possível escolha é considerar as falhas como não curados e as censuras como curados.

- 2. (Passo M - maximização de $L_{1}$ ) Considerando os valores de $p_{i}$ como resposta e $\mathbf{Z}_{2 i}$ como covariáveis, ajustar um modelo linear generalizado com distribuição binomial para obter as estimativas de $\boldsymbol{\alpha}^{1}$.

\footnotetext{
${ }^{1}$ Neste passo, em geral utiliza-se a função de ligação logito e ajusta-se um modelo de regressão logística. Outras funçoes de ligação e outros modelos como, por exemplo, modelos não lineares aditivos, também poderiam ser considerados.
} 
- 3. (Passo M - maximização de $L_{2}$ ) Ajustar um modelo de riscos proporcionais de Cox ${ }^{2}$ considerando o vetor de covariáveis $\mathbf{Z}_{1 i}$ e uma covariável adicional $\log p_{i}$ com coeficiente fixo igual a 1 e assim obter as estimativas de $\boldsymbol{\beta}$.

- 4. Com base no modelo ajustado no passo 3, obter as estimativas de $S_{N C}^{0}{ }^{3}$ para todos os tempos observados, usando (2.10). Substituir os valores por zero nos casos em que o tempo observado exceder o maior tempo de falha.

- 5. (Passo E - esperança de $\left.b_{i}\right)$ A partir das estimativas $\hat{\boldsymbol{\alpha}}, \hat{\boldsymbol{\beta}}$ e $\hat{S}_{N C}^{0}\left(t_{i}\right)$, atualizar as estimativas dos $p_{i}$ 's usando a fórmula dada em (2.5).

- 6. Repetir os passos 2, 3, 4 e 5 até a convergência das estimativas de $\boldsymbol{\alpha}$ e $\boldsymbol{\beta}$.

- 7. Aplicar o método de bootstrap para obter os erros padrão das estimativas dos parâmetros.

Este procedimento pode ser implementado em qualquer programa estatístico que contenha funções para bootstrap e para ajuste de modelos lineares generalizados, modelo de Cox e suas extensões. Peng (2003b) disponibilizou um programa para o software S-Plus chamado semicure, que fornece estimativas de $\boldsymbol{\alpha}$, para o modelo logístico referente à fração de cura, e de $\boldsymbol{\beta}$, para o modelo de Cox referente à distribuição dos tempos de falha. Convém ressaltar que a função semicure não contempla o passo 7 com o procedimento bootstrap para a obtenção das estimativas dos erros.

\footnotetext{
${ }^{2}$ No modelo de Cox, estratificações e covariáveis dependentes do tempo podem ser consideradas, se necessário.

${ }^{3} \mathrm{Na}$ maioria dos programas estatísticos, na função que ajusta o modelo de Cox, existe uma opção para calcular as estimativas da função de sobrevivência padrão $S^{0}(t)$.
} 
Na função semicure, o critério de convergência adotado é

$$
\max \left\{\max _{0 \leq i \leq r_{2}}\left|\frac{\boldsymbol{\alpha}_{i}^{(k+1)}-\boldsymbol{\alpha}_{i}^{(k)}}{\boldsymbol{\alpha}_{i}^{(k+1)}}\right| ; \max _{1 \leq j \leq r_{1}}\left|\frac{\boldsymbol{\beta}_{j}^{(k+1)}-\boldsymbol{\beta}_{j}^{(k)}}{\boldsymbol{\beta}_{j}^{(k+1)}}\right|\right\}<0,0001
$$

$\operatorname{com} \boldsymbol{\alpha}=\left(\alpha_{0}, \alpha_{1}, \ldots, \alpha_{r_{2}}\right)$ e $\boldsymbol{\beta}=\left(\beta_{1}, \ldots, \beta_{r_{1}}\right)$ representando, respectivamente, os parâmetros do modelo logístico para a probabilidade de cura e os parâmetros do modelo de Cox pra os tempos de sobrevivência. 


\section{Capítulo 3}

\section{Método da ponderação em modelos com fração de cura}

A análise de casos completos (ACC), freqüentemente utilizada na prática, consiste em considerar apenas os casos sem omissão em todas as covariáveis e, conforme mencionado anteriormente, pode levar à exclusão de grande parte da amostra. Com o intuito de amenizar o impacto dessa exclusão, uma possível abordagem é ponderar as observações completas, atribuindo maior importância a grupos sub-representados, na tentativa de corrigir eventuais desproporcionalidades na subamostra de casos completos em relação à amostra original.

A idéia da ponderação foi proposta originalmente por Binder (1992) no contexto de levantamentos amostrais (survey data) nos quais os indivíduos que compõem a amostra apresentam diferentes probabilidades de seleção e, são ponderados de acordo com estas quantidades (sampling weights). No caso de dados omissos, os indivíduos selecionados são aqueles que não apresentam omissão em nenhuma das covariáveis e a probabilidade de seleção pode estar relacionada tanto ao mecanismo de omissão quanto à taxa de cura.

A proposta deste trabalho é utilizar a abordagem de ponderação dos casos completos para lidar com a omissão em situações em que a ocorrência de dados omissos está 
relacionada com uma maior ocorrência do evento de interesse. Mais especificamente, será considerada a situação em que a omissão está associada a uma menor fração de curados.

Neste capítulo são apresentados os modelos com fração de cura ponderados considerando a abordagem paramétrica (família Weibull) e semiparamétrica (modelo de Cox). Dois tipos de ponderação são propostos: pesos com base nas probabilidades de omissão e pesos com base nas probabilidades de cura.

\subsection{Modelos ponderados}

Para descrever a metodologia associada à ponderação de observações completas, suponha válido um modelo estatístico (paramétrico ou semiparamétrico) a partir do qual constrói-se a função de verossimilhança $L_{n}(\boldsymbol{\theta})$. O parâmetro $\boldsymbol{\theta}$ é estimado pelo método da máxima verossimilhança, que consiste em resolver a equação de estimação $\mathbf{U}_{n}(\boldsymbol{\theta})=\sum_{i=1}^{n} \mathbf{U}_{i}(\boldsymbol{\theta})=\mathbf{0}$.

Nos modelos ponderados, a idéia é inserir pesos $w_{i}$ na contribuição dos indivíduos dentro da função escore, obtendo uma função escore ponderada $\mathbf{U}_{n, w}(\boldsymbol{\theta})=\sum_{i=1}^{n} w_{i} \mathbf{U}_{i}(\boldsymbol{\theta})$.

Para os levantamentos amostrais abordados por Binder (1992), os pesos são determinísticos pois correspondem às probabilidades de seleção, que por sua vez são definidas a partir do planejamento amostral. No entanto, para a situação em que se tem dados com omissão, os pesos não são conhecidos e precisam ser estimados com base em algum modelo. Neste caso, adota-se um modelo paramétrico em que $\gamma$ representa um vetor de parâmetros desconhecidos. Conseqüentemente, a função escore ponderada depende também da parametrização para os pesos, e pode ser expressa em função do novo vetor de parâmetros, ou seja, 


$$
\mathbf{U}_{n, w}(\boldsymbol{\theta}, \boldsymbol{\gamma})=\sum_{i=1}^{n} w_{i}(\boldsymbol{\gamma}) \mathbf{U}_{i}(\boldsymbol{\theta})
$$

Embora teoricamente seja possível estimar os parâmetros $\boldsymbol{\gamma}$ e $\boldsymbol{\theta}$ simultaneamente, como o interesse é utilizar a amostra total para estimação dos pesos, o processo de estimação é realizado em duas etapas. Portanto, para estimar o parâmetro $\boldsymbol{\theta}$, obtémse separadamente $\hat{\gamma}$, a estimativa de máxima verossimilhança de $\gamma$ com base no modelo para $w_{i}(\boldsymbol{\gamma})$, e a partir daí obtém-se $\hat{\boldsymbol{\theta}}$ como solução da equação $\mathbf{U}_{n, w}(\boldsymbol{\theta}, \hat{\boldsymbol{\gamma}})=\mathbf{0}$.

O primeiro passo é especificar um modelo para os pesos. A seguir são apresentadas duas propostas de ponderação. Os métodos convencionais comumente utilizados em dados de sobrevivência, além de desperdiçarem todos os dados dos casos incompletos, assumem que a omissão é completamente aleatória (MCAR), o que muitas vezes não é razoável na prática. Na primeira proposta, assume-se que a omissão depende apenas das covariáveis que são observadas para todos os indivíduos e dos tempos de falha ou censura. Na segunda proposta, considera-se que a omissão está relacionada às taxas de cura e que estas devem ser levadas em conta na obtenção dos pesos, que é o caso do conjunto de dados discutido no Capítulo 1.

\subsubsection{Ponderação com base nas probabilidades de omissão}

Neste caso, utilizam-se pesos que correspondem ao recíproco das probabilidades estimadas de não omissão, modeladas por um modelo logístico.

Seja

$$
R_{i}=\left\{\begin{array}{l}
1, \quad \text { se a observação } i \text { é completa (sem dados omissos) } \\
0, \quad \text { se a observação } i \text { é incompleta (com dados omissos) }
\end{array}\right.
$$

Convém observar que na análise de casos completos (ACC), a função escore de fato 
utilizada na estimação dos parâmetros é:

$$
\mathbf{U}_{n}(\boldsymbol{\theta})=\sum_{i=1}^{n} R_{i} \mathbf{U}_{i}(\boldsymbol{\theta}),
$$

o que corresponde a uma função escore ponderada com pesos determinísticos iguais a 0 para os indivíduos com omissão e 1 para os casos completos.

Seja $\xi_{i}$ a probabilidade de o indivíduo $i$ ter dados completos $\left(\xi_{i}=P\left(R_{i}=1\right)\right)$. Neste trabalho, assumimos que a omissão depende apenas do que é observado para todos os indivíduos da amostra, isto é,

$$
\xi_{i}=P\left(R_{i}=1 \mid X_{i}, \Delta_{i}, \mathbf{Z}_{i, \text { comp }}\right), \quad i=1, \ldots, n
$$

em que $X_{i}$ é o mínimo entre o tempo de falha e o tempo de censura, $\Delta_{i}$ é o indicador de falha e $\mathbf{Z}_{i, \text { comp }}$ é o subvetor do vetor de covariáveis $\mathbf{Z}_{i}$ contendo apenas as covariáveis completamente observadas (sem dados omissos).

Com base nessa suposição, é natural estimar as probabilidades de dados completos por um modelo de regressão logística, considerando como resposta a variável $R_{i}$ e como covariáveis todas aquelas com dados completos para todos os indivíduos da amostra, incluindo como covariáveis os tempos de falha (ou censura) e os indicadores de falha.

A partir do modelo logístico

$$
\xi_{i}(\boldsymbol{\gamma})=\frac{\mathrm{e}^{\left(\boldsymbol{Z}_{i}^{* \prime} \boldsymbol{\gamma}\right)}}{1+\mathrm{e}^{\left(\boldsymbol{Z}_{i}^{* \prime} \boldsymbol{\gamma}\right)}}
$$

em que $\mathbf{Z}_{i}^{* \prime}=\left(\mathbf{Z}_{i, c o m p}, X_{i}, \Delta_{i}\right)$, o estimador de máxima verossimilhança de $\boldsymbol{\gamma}$ é obtido maximizando a função de verossimilhança 


$$
L(\boldsymbol{\gamma})=\prod_{i=1}^{n}\left(\xi_{i}(\gamma)\right)^{R_{i}}\left(1-\xi_{i}(\gamma)\right)^{\left(1-R_{i}\right)}
$$

Dessa forma, a probabilidade de que o $i$-ésimo indivíduo tenha dados completos (não omissão) é estimada por

$$
\hat{\xi}_{i}=\xi_{i}(\hat{\gamma})=\frac{\mathrm{e}^{\left(\mathbf{Z}_{i}^{* \prime} \hat{\gamma}\right)}}{1+\mathrm{e}^{\left(\mathbf{Z}_{i}^{* \prime} \hat{\gamma}\right)}}
$$

em que $\hat{\gamma}$ é o estimador de máxima verossimilhança de $\gamma$, obtido da forma usual (ver McCullagh \& Nelder (1989)).

Vale notar que, como o modelo logístico inclui apenas as covariáveis completamente observadas, este método aproveita boa parte da informação disponível para os indivíduos incompletos, que seria totalmente desperdiçada na ACC. No modelo final para estimar $\boldsymbol{\theta}$ são utilizados apenas os casos completos, sendo que aqueles mais "parecidos" com indivíduos com dados omissos terão maior peso na análise.

A idéia do método é dar maior peso aos casos que são semelhantes àqueles que foram excluídos devido a omissões em outras covariáveis, utilizando assim o recíproco das probabilidades estimadas com os dados completos, ou seja, considerando $w_{i}(\hat{\gamma})=$ $1 / \xi_{i}(\hat{\gamma})$. Dessa forma, as estimativas do parâmetro $\boldsymbol{\theta}$ são obtidas a partir da equação de estimação ponderada

$$
\mathbf{U}_{n, w}(\boldsymbol{\theta}, \hat{\gamma})=\sum_{i=1}^{n} \frac{R_{i}}{\xi_{i}(\hat{\gamma})} \mathbf{U}_{i}(\boldsymbol{\theta})=\mathbf{0}
$$

em que $\xi_{i}(\hat{\gamma})$ é dado por (3.1).

A proposta de ponderar os casos completos pelo recíproco da probabilidade de não omissão foi abordada originalmente por Robins et al. (1994). Este método foi abordado 
por Pugh, Robbins, Lipsitz \& Harrington (1993) para modelos de riscos proporcionais de Cox.

\subsubsection{Ponderação com base nas probabilidades de cura}

Uma limitação do método proposto na seção anterior é que, para a estimação dos pesos, não se leva em conta que os dados contêm uma proporção de curados e que esta característica pode estar relacionada com a omissão de informações. Isto parece incoerente, uma vez que no modelo final ponderado, parte-se do pressuposto que existem duas subpopulações (curados e não curados) com prognósticos diferenciados, e que aqueles com pior prognóstico apresentam mais dados omissos.

A partir da idéia de ponderar os casos completos segundo a probabilidade de não omissão, pode-se pensar em outra proposta de ponderação com base nas probabilidades de cura, que leve em conta a presença de curados e a relação entre cura e omissão.

Nos dados que serviram de motivação para este trabalho, para várias das covariáveis presentes, os pacientes com dados completos apresentam maiores tempos de sobrevivência, que por sua vez podem estar relacionados a maiores probabilidades de cura. Logo, é natural procurar incorporar esta informação no tratamento das omissões, levando-se em consideração que quanto menor a probabilidade de cura, maior a probabilidade de omissão. Desta forma, em vez de aumentar a participação dos casos semelhantes àqueles com valores omissos, procura-se aumentar a participação dos casos com menor probabilidade de cura, assumindo-se que existe uma relação entre cura e não omissão. Assim como no caso anterior, é atribuída maior importância àqueles indivíduos que teoricamente teriam mais dificuldades em ser selecionados para a sub-amostra de casos completos, porém considerando que estas dificuldades estão relacionadas à taxa de cura. 
Neste caso, a probabilidade de cura também é definida por um modelo logístico, porém que faz parte de um modelo de mistura. Como assume-se que a omissão está relacionada à cura, o modelo para estimar as probabilidades de cura pode incluir o indicador de omissão (representado pela variável $R_{i}$ ) como mais uma covariável, além daquelas completamente observadas.

Considere um modelo de fração de cura não ponderado em que, tanto no termo de sobrevivência quanto no termo de cura, são incluídas apenas as covariáveis completamente observadas para todos os indivíduos e os indicadores de omissão. Seja $\xi_{i}$ a probabilidade de o indivíduo $i$ ser curado que é expressa em função do vetor de covariáveis $\mathbf{Z}_{i}^{*}$ e do correspondente vetor de parâmetros $\gamma$. A estimativa de máxima verossimilhança $\hat{\gamma}$ é obtida iterativamente de acordo com o processo de estimação descrito no Capítulo 2, seja no modelo Weibull ou no semiparamétrico ${ }^{1}$. Então, dado $\hat{\gamma}$, a probabilidade de cura do indivíduo $i$ é estimada por

$$
\hat{\xi}_{i}=\xi_{i}(\hat{\gamma})=\frac{1}{1+\mathrm{e}^{\left(\boldsymbol{Z}_{i}^{* \prime} \hat{\gamma}\right)}}
$$

na qual $\mathbf{Z}_{i}^{* \prime}=\left(\mathbf{Z}_{i, \text { comp }}, R_{i}\right)^{\prime}, \mathbf{Z}_{i, \text { comp }}$ é o vetor de covariáveis sem omissão.

Logo, utilizam-se covariáveis sem dados omissos e os indicadores de omissão para ajustar um modelo com fração de cura usual sem ponderação e as estimativas das probabilidades de cura são utilizadas como pesos no modelo final com os casos completos.

Para atribuir maior peso aos indivíduos com dados completos e que tenham menor probabilidade de cura, toma-se como peso o inverso da probabilidade de cura, ou seja, $w_{i}=1 / \xi_{i}(\hat{\gamma})$. Assim como na seção anterior, a informação dos indivíduos com dados omissos é aproveitada na estimação dos pesos e os parâmetros de interesse são obtidos por $(3.2)$, porém $\operatorname{com} \xi_{i}(\hat{\gamma})$ correspondendo à estimativa da probabilidade de cura.

\footnotetext{
${ }^{1}$ Convem notar que no Capítulo 2 o modelo está especificado em termos da probabilidade de não cura $\pi_{i}=1-\xi_{i}$.
} 
Neste método também é utilizado o critério de "parecença" no sentido que os indivíduos mais parecidos com aqueles que foram excluídos por apresentarem omissão receberão maior peso na análise. No entanto, considera-se que a omissão é devida à não cura e assim assume-se que o peso pela omissão corresponde ao peso pela não cura.

O esquema a seguir resume as duas propostas de ponderação.

\section{SUBAMOSTRA DE CASOS COMPLETOS MAIOR SOBREVIVÊNCIA}

(maior taxa de cura quando comparada à subamostra de casos incompletos)

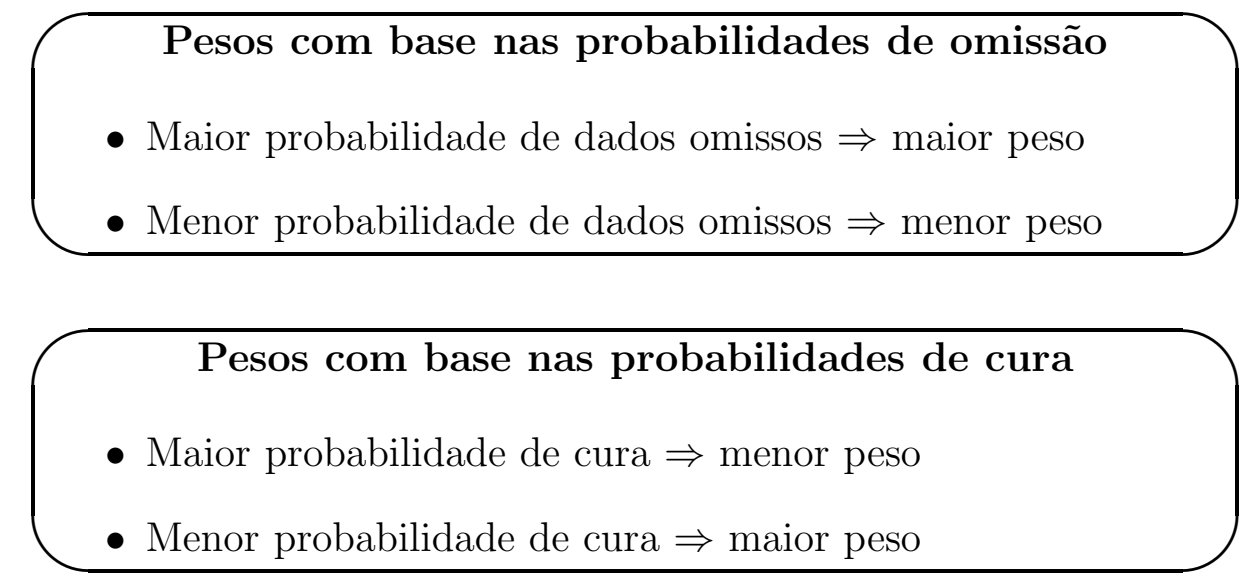

Em resumo, em ambos os métodos estima-se os pesos considerando-se todos os indivíduos da amostra (com e sem dados omissos). Com os pesos estimados, ajusta-se um novo modelo com fração de cura ponderado, considerando-se apenas os indivíduos com dados completos.

Embora os casos com omissão continuem a não ser considerados no modelo final, parte da informação contida nestes casos é utilizada para a estimação dos pesos. Esta informação usualmente é desperdiçada na análise de casos completos (ACC). 


\subsection{Modelos com fração de cura ponderados}

A metodologia proposta neste trabalho consiste em estender o procedimento proposto por Robins et al. (1994) e Pugh et al. (1993) para modelos com fração de cura. O processo de estimação é dividido em duas etapas: uma para a estimação dos pesos, que corresponde ao ajuste de um modelo não ponderado e outra que ajusta um modelo ponderado, assumindo funções das estimativas obtidas do primeiro modelo como pesos. Nos modelos para estimação dos pesos (modelo 1) são incluídas apenas as covariáveis sem omissão e, portanto, todos os indivíduos são utilizados (com dados completos e incompletos). Já no modelo final ponderado (modelo 2), são incluídas todas as covariáveis de interesse e, com isso, apenas a subamostra de casos completos é considerada.

Para estimar os pesos, pode-se adotar um modelo de regressão logística usual no caso de ponderação pela omissão, ou um modelo com fração de cura não ponderado no caso de ponderação pela fração de cura. Como o interesse é analisar dados com proporção de curados, o segundo modelo corresponde sempre a um modelo com fração de cura ponderado, utilizando como pesos $w_{i}=1 / \xi_{i}(\hat{\gamma})$ nos quais $\xi_{i}(\hat{\gamma})$ podem ser as estimativas das probabilidades de dado completo (não omissão) ou das probabilidades de cura.

\subsubsection{Ponderação na família Weibull}

No Capítulo 2, foi visto que a função escore referente ao modelo Weibull com fração de cura pode ser escrita como

$$
\mathbf{U}_{n}(\boldsymbol{\theta})=\sum_{i=1}^{n} \mathbf{Z}_{i} \mathbf{s}_{i}(\boldsymbol{\theta}),
$$

em que $\mathbf{Z}_{i}$ é uma matriz não estocástica que envolve os vetores $\mathbf{Z}_{1 i}$ e $\mathbf{Z}_{2 i}$ de covariáveis incluídas na parte de sobrevivência e de cura, respectivamente, e $\mathbf{s}_{i}(\boldsymbol{\theta})$ é um vetor de 
dimensão $3 \times 1$, cujos elementos são funções dos parâmetros de interesse $\delta, \lambda_{i}$ e $\pi_{i}$ especificados na Seção 2.2.

Logo, no modelo Weibull com fração de cura ponderado, que leva em conta apenas os casos completos, a função escore considerada é

$$
\mathbf{U}_{n, w}(\boldsymbol{\theta})=\sum_{i=1}^{n} R_{i} w_{i} \mathbf{Z}_{i} \mathbf{s}_{i}(\boldsymbol{\theta})
$$

na qual $w_{i}$ é o peso atribuído ao indivíduo $i$, que pode ser o recíproco da probabilidade de dado completo estimada por um modelo de regressão logística ou o recíproco da probabilidade de cura estimada por um modelo Weibull com fração de cura.

O estimador (ponderado) para o parâmetro $\boldsymbol{\theta}$ é obtido pela solução da equação $\mathbf{U}_{n, w}\left(\boldsymbol{\theta}_{w}\right)=0$, que é resolvida iterativamente pelo algoritmo de Newton-Raphson.

Assumindo que os pesos $w_{i}$ não dependem dos parâmetros $\pi_{i}, \lambda_{i}$ e $\delta$, a matriz de informação pode ser escrita como

$$
\mathbf{F}_{n, w}(\boldsymbol{\theta})=-\frac{\partial \mathbf{U}_{n, w}(\boldsymbol{\theta})}{\partial \boldsymbol{\theta}}=\sum_{i=1}^{n} R_{i} w_{i} \mathbf{Z}_{i} \mathcal{F}_{i}(\boldsymbol{\theta}) \mathbf{Z}_{i}^{\prime}
$$

em que $\mathcal{F}_{i}(\boldsymbol{\theta})$ é a matriz 3 x 3 simétrica descrita no Capítulo 2, cujas componentes são detalhadas no Apêndice A.

Assim como no modelo não ponderado, a partir de um valor inicial para o parâmetro $\boldsymbol{\theta}$, a estimativa de $\boldsymbol{\theta}$ é atualizada por

$$
\boldsymbol{\theta}_{K+1}=\boldsymbol{\theta}_{K}+\mathbf{F}_{n, w}^{-1}\left(\boldsymbol{\theta}_{K}\right) \mathbf{U}_{n, w}\left(\boldsymbol{\theta}_{K}\right)
$$

em que $\boldsymbol{\theta}_{K}$ e $\boldsymbol{\theta}_{K+1}$ são as estimativas de $\boldsymbol{\theta}$ nos passos $K$ e $K+1$. A convergência é atingida quando $\frac{\left\|\boldsymbol{\theta}_{K+1}-\boldsymbol{\theta}_{K}\right\|}{\left\|\boldsymbol{\theta}_{K}\right\|}<\epsilon$, com $\epsilon$ igual a um valor pré especificado. 


\subsubsection{Ponderação no modelo semiparamétrico}

No modelo semiparamétrico com fração de cura, o vetor de parâmetros de interesse é $\boldsymbol{\theta}=(\boldsymbol{\alpha}, \boldsymbol{\beta})$ em que $\boldsymbol{\alpha}$ está associado à fração de cura e $\boldsymbol{\beta}$ à sobrevivência. Conforme foi visto no Capítulo 2, supondo que a cura é observável, a função de verossimilhança completa pode ser escrita como

$$
L_{n}(\boldsymbol{\theta})=L_{1}(\boldsymbol{\alpha} ; \mathbf{b}) L_{2}\left(\boldsymbol{\beta} ; \mathbf{S}_{N C}^{0} ; \mathbf{b}\right)=\prod_{i=1}^{n} L_{1 i}\left(\boldsymbol{\alpha} ; b_{i}\right) \prod_{i=1}^{n} L_{2 i}\left(\boldsymbol{\beta} ; S_{N C}^{0}\left(t_{i}\right) ; b_{i}\right)
$$

em que $\mathbf{b}=\left(b_{1}, \ldots, b_{n}\right)$ é vetor dos indicadores de não cura, $L_{1}(\boldsymbol{\alpha} ; \mathbf{b})$ é a função de verossimilhança de um modelo logístico usual e $L_{2}\left(\boldsymbol{\beta} ; \mathbf{S}_{N C}^{0} ; \mathbf{b}\right)$ é a função de verossimilhança completa para dados de sobrevivência sob o modelo de Cox.

Tomando o logaritmo desta função e derivando em relação a $\boldsymbol{\theta}$, obtém-se a função escore

$$
\begin{aligned}
\mathbf{U}_{n}(\boldsymbol{\theta}) & =\frac{\partial \log L_{n}(\boldsymbol{\theta})}{\partial \boldsymbol{\theta}}=\sum_{i=1}^{n} \frac{\partial \log L_{1 i}\left(\boldsymbol{\alpha} ; b_{i}\right)}{\partial \boldsymbol{\theta}}+\sum_{i=1}^{n} \frac{\partial \log L_{2 i}\left(\boldsymbol{\beta} ; S_{N C}^{0}\left(t_{i}\right) ; b_{i}\right)}{\partial \boldsymbol{\theta}} \\
& =\sum_{i=1}^{n}\left[\mathbf{U}_{1 i}\left(\boldsymbol{\alpha} ; b_{i}\right)+\mathbf{U}_{2 i}\left(\boldsymbol{\beta} ; S_{N C}^{0}\left(t_{i}\right) ; b_{i}\right)\right]
\end{aligned}
$$

Portanto, a contribuição do indivíduo $i$ para a função escore corresponde à soma das contribuições individuais nas funções escore do termo logístico e do termo de sobrevivência.

Considerando apenas os casos completos e inserindo os pesos individuais $w_{i}$, a função escore ponderada pode ser escrita como a soma das funções escores ponderadas

$$
\mathbf{U}_{n, w}(\boldsymbol{\theta})=\mathbf{U}_{n, w 1}(\boldsymbol{\alpha} ; \mathbf{b})+\mathbf{U}_{n, w 2}\left(\boldsymbol{\beta} ; \mathbf{S}_{N C}^{0} ; \mathbf{b}\right)
$$


$\operatorname{com} \mathbf{U}_{n, w 1}(\boldsymbol{\alpha} ; \mathbf{b})=\sum_{i=1}^{n} R_{i} w_{i} \mathbf{U}_{1 i}\left(\boldsymbol{\alpha}, b_{i}\right) \quad \mathrm{e}$

$\mathbf{U}_{w 2}\left(\boldsymbol{\beta} ; \mathbf{S}_{N C}^{0} ; \mathbf{b}\right)=\sum_{i=1}^{n} R_{i} w_{i} \mathbf{U}_{2 i}\left(\boldsymbol{\beta} ; S_{N C}\left(t_{i}\right) ; b_{i}\right)$.

Como a maximização é feita separadamente, para ajustar um modelo com fração de cura semiparamétrico ponderado basta considerar, no passo $\mathrm{M}$ do processo de estimação, um modelo logístico ponderado e um modelo de sobrevivência ponderado. Vale notar que, embora $L_{2}\left(\boldsymbol{\beta}, \mathbf{S}_{N C}, \mathbf{b}\right)$ seja a função de verossimilhança completa para dados de sobrevivência sob o modelo de Cox, no processo de estimação considerase o procedimento usual de maximização da verossimilhança parcial de Cox e $\mathbf{S}_{N C}$ é estimado pela fórmula de Breslow modificada.

No método da ponderação para tratar dados omissos, os pesos $w_{i}$ são estimados por um modelo paramétrico $w_{i}(\gamma)$. Apesar disso, assim como no modelo paramétrico, para a descrição dos modelos ponderados assume-se que os pesos são conhecidos e não dependem dos parâmetros a serem estimados (parâmetro $\boldsymbol{\alpha}$ do modelo logístico e parâmetro $\boldsymbol{\beta}$ do modelo de sobrevivência).

\section{Modelo logístico ponderado}

Para o modelo logístico, assume-se que os pesos $w_{i}$ não dependem do parâmetro $\boldsymbol{\alpha}$ e a função escore ponderada é dada pela expressão:

$$
\mathbf{U}_{n, w 1}(\boldsymbol{\alpha} ; \mathbf{b})=\sum_{i=1}^{n} R_{i} w_{i} \frac{\partial}{\partial \boldsymbol{\alpha}}\left\{b_{i} \log \pi_{i}(\boldsymbol{\alpha})+\left(1-b_{i}\right) \log \left(1-\pi_{i}(\boldsymbol{\alpha})\right)\right\}
$$

Se os valores de $\mathbf{b}=\left\{b_{i} ; i=1, \ldots, n\right\}$ fossem conhecidos, o estimador de máxima verossimilhança ponderado $\boldsymbol{\alpha}_{w}$ seria obtido pela solução da equação $\mathbf{U}_{n, w 1}(\boldsymbol{\alpha} ; \mathbf{b})=\mathbf{0}$.

No algoritmo EM descrito anteriormente, a verossimilhança é maximizada substituindo $b_{i}$ por uma estimativa de sua esperança condicional $p_{i}$, calculada no passo E. 
Logo, cada iteração $k$, o passo M consiste em atualizar a estimativa $\boldsymbol{\alpha}_{w}^{(k)}$ resolvendo a equação $\mathbf{U}_{n, w 1}\left(\boldsymbol{\alpha}_{w}^{(k+1)} ; \mathbf{p}^{(k)}\right)=\mathbf{0}$, em que $\mathbf{p}^{(\mathbf{k})}=\left(p_{1}^{(k)}, p_{2}^{(k)}, \ldots, p_{n}^{(k)}\right)$ são as estimativas dos $p_{i}$ 's calculadas na $k$-ésima iteração.

\section{Modelo de Cox ponderado}

No modelo de sobrevivência, o que é de fato maximizado para estimar o parâmetro $\boldsymbol{\beta}$ é a função de verossimilhança parcial (Cox (1975)). Para o modelo de Cox, a ponderação tem algumas particularidades. Usualmente, nos modelos de Cox ponderados, a função escore não corresponde apenas à soma ponderada da função escore usual $\sum_{i} w_{i} \mathbf{U}_{i}$, pois as ponderações são incorporadas também nos termos da soma, isto é, $\mathbf{U}_{w}=\sum_{i} w_{i} \mathbf{U}_{w i}$ em que $\mathbf{U}_{w i}$ é uma função do parâmetro de interesse, dos dados observados e dos pesos individuais.

Considera-se aqui o modelo de Cox ponderado descrito sob o enfoque de processos de contagem (Andersen, Borgan, Gill \& Keiding (1993)).

Sejam $T_{1}, T_{2}, \ldots, T_{n}$ os tempos de falha, $C_{1}, C_{2}, \ldots, C_{n}$ os tempos de censura e defina $Y_{i}(t)=I_{\left\{\min \left(T_{i}, C_{i}\right) \geq t\right\}}$ como o indicador de risco do indivíduo $i$ no instante $t$. Considere $d N_{i}(t)=N_{i}(t)-N_{i}(t-d t)$ o incremento de um processo de contagem $N_{i}(t)=I_{\left\{\min \left(T_{i}, C_{i}\right) \leq t, \Delta_{i}=1\right\}}$, que corresponde ao indicador de falha para o indivíduo $i$.

Dados os vetores de covariáveis $\mathbf{Z}_{i}, i=1, \ldots, n$, a função escore relativa à verossimilhança parcial, escrita em termos de processos de contagem, é:

$$
\mathbf{U}_{n}(\boldsymbol{\beta})=\sum_{i=1}^{n} \int_{0}^{\infty}\left\{\mathbf{Z}_{i}-\overline{\mathbf{Z}}(\boldsymbol{\beta}, s)\right\} d N_{i}(s)
$$

em que $\overline{\mathbf{Z}}(\boldsymbol{\beta}, t)=\frac{\sum_{j=1}^{n} Y_{j}(t) \mathbf{Z}_{j} \mathrm{e}^{\mathbf{Z}_{j}^{\prime} \boldsymbol{\beta}}}{\sum_{j=1}^{n} Y_{j}(t) \mathrm{e}^{\mathbf{Z}_{j}^{\prime} \boldsymbol{\beta}}}$.

Embora, em geral, a função escore de modelos ponderados corresponda simples- 
mente à soma ponderada da função escore, no modelo de Cox ponderado (Therneau \& Grambsch (2000)), usualmente os pesos individuais $w_{i}$ também são incorporados na soma em $\overline{\mathbf{Z}}(\boldsymbol{\beta}, t)$ e a função escore ponderada, considerando somente os casos completos, é expressa por:

$$
\mathbf{U}_{n, w}(\boldsymbol{\beta})=\sum_{i=1}^{n} \int_{0}^{\infty} R_{i} w_{i}\left\{\mathbf{Z}_{i}-\overline{\mathbf{Z}}_{w}(\boldsymbol{\beta}, s)\right\} d N_{i}(s)
$$

$\operatorname{com} \overline{\mathbf{Z}}_{w}(\boldsymbol{\beta}, t)=\frac{\sum_{j=1}^{n} R_{j} w_{j} Y_{j}(t) \mathbf{Z}_{j} \mathrm{e}^{\mathbf{Z}_{j}^{\prime} \boldsymbol{\beta}}}{\sum_{j=1}^{n} R_{j} w_{j} Y_{j}(t) \mathrm{e}^{\mathbf{Z}_{j}^{\prime} \boldsymbol{\beta}}}$. Assim, o estimador de máxima verossimilhança parcial ponderado $\hat{\boldsymbol{\beta}}_{w}$ é obtido resolvendo-se a equação $\mathbf{U}_{n, w}(\boldsymbol{\beta})=\mathbf{0}$.

Voltando aos modelos com fração de cura, no processo de estimação usando o algoritmo EM, a função de verossimilhança parcial de Cox maximizada no passo M corresponde à função usual com a inserção de $\log b_{i}$ como mais uma covariável com coeficiente fixo igual a 1 e considerando as estimativas da esperança condicional $p_{i}$ no lugar de $b_{i}$. Portanto, no modelo ponderado, dadas as estimativas $p_{i}^{(k)}$, obtidas na $k$-ésima iteração, o passo $\mathrm{M}$ consiste em atualizar a estimativa $\boldsymbol{\beta}_{w}^{(k)}$ resolvendo a equação

$$
\mathbf{U}_{n, w 2}\left(\boldsymbol{\beta}_{w}^{(k+1)} ; \mathbf{p}^{(k)}\right)=\sum_{i=1}^{n} \int_{0}^{\infty} R_{i} w_{i}\left\{\mathbf{Z}_{i}-\overline{\mathbf{Z}}_{w}^{*}\left(\boldsymbol{\beta}_{w}^{(k+1)}, \mathbf{p}^{(k)}, s\right)\right\} d N_{i}(s)=\mathbf{0}
$$

$\operatorname{com} \overline{\mathbf{Z}}_{w}^{*}\left(\boldsymbol{\beta}_{w}, \mathbf{p}, t\right)=\frac{\sum_{j=1}^{n} R_{j} w_{j} Y_{j}(t) \mathbf{Z}_{j} p_{j} \mathrm{e}^{\mathbf{Z}_{j}^{\prime} \boldsymbol{\beta}_{w}}}{\sum_{j=1}^{n} R_{j} w_{j} Y_{j}(t) p_{j} \mathrm{e}^{\mathbf{Z}_{j}^{\prime} \boldsymbol{\beta}_{w}}}$

No modelo ponderado, a função de sobrevivência padrão $\mathbf{S}_{N C, w}^{0}$ é estimada pela fórmula de Breslow modificada (expressão (2.10)) considerando $\hat{\boldsymbol{\beta}}_{w}$ no lugar de $\hat{\boldsymbol{\beta}}$. Isto é, a cada iteração, $\mathbf{S}_{N C, w}^{0(k+1)}$ é atualizada a partir de $\boldsymbol{\beta}_{w}^{(k+1)}$.

Portanto, para ajustar um modelo semiparamétrico com fração de cura ponderado, utiliza-se o algoritmo EM descrito no Capítulo 2. Porém, as funções escore correspon- 
dentes a $L_{1}$ e $L_{2}$ utilizadas no passo $\mathrm{M}$ são substituídas pelas funções escore ponderadas $\mathbf{U}_{n, w 1}$ e $\mathbf{U}_{n, w 2}$. Isto significa que, a cada passo do processo iterativo, ajusta-se um modelo logístico ponderado e um modelo de Cox ponderado para atualização das estimativas de $\boldsymbol{\alpha}_{w}, \boldsymbol{\beta}_{w}$ e $\mathbf{S}_{N C, w}^{0}$.

\subsection{Obtenção dos estimadores ponderados}

Os passos a seguir resumem o método proposto neste capítulo.

1. Estimam-se os pesos utilizando a amostra total. Nesta etapa, utiliza-se um modelo de regressão logística usual ou um modelo com fração de cura, considerando apenas covariáveis sem dados omissos. A partir do modelo $\xi_{i}(\gamma)$ assumido para as probabilidades de não omissão (ou de cura), obtem-se o estimador de máxima verossimilhança $\hat{\gamma}$ e estimam-se as probabilidades por $\xi_{i}(\hat{\gamma})$.

2. Com as estimativas obtidas no passo 1, ajusta-se um modelo com fração de cura ponderado utilizando-se apenas a sub-amostra de casos completos. Neste caso, os pesos $w_{i}$ são assumidos como parâmetros fixos iguais a $R_{i} / \xi_{i}(\hat{\gamma})$, isto é, o estimador de máxima verossimilhança ponderado, $\hat{\boldsymbol{\theta}}_{w}$, é aquele que maximiza uma função de verossimilhança perfilada $L_{n, w}(\boldsymbol{\theta}, \hat{\gamma})$ e é obtido resolvendo a equação $\mathbf{U}_{n, w}(\boldsymbol{\theta}, \hat{\gamma})=\mathbf{0}$ 


\section{Capítulo 4}

\section{Aplicações}

Com o intuito de ilustrar os métodos propostos neste trabalho, foi realizado um estudo de simulação com base nos dados de insuficiência cardíaca apresentados no Capítulo 1. A partir do banco de dados original de 1101 pacientes, foi selecionada uma sub-amostra de 898 pacientes com dados completos em quatro covariáveis: sexo (1=masculino e $0=$ feminino), idade (em anos), doença de Chagas $(1=$ presente e $0=$ ausente) e fração de ejeção (em \%).

As variáveis sexo e idade foram escolhidas pois, além de estarem disponíveis para todos os indivíduos, em estudos da área médica estas são variáveis de controle que normalmente são incluídas nos modelos finais, mesmo que não sejam estatisticamente significantes. A doença de Chagas foi um dos principais fatores identificados na análise de Freitas et al. (2005), sendo que pacientes chagásicos apresentam pior prognóstico do que não chagásicos. Por último, a fração de ejeção usualmente é um indicador importante de gravidade (quanto maior a fração de ejeção melhor a condição do paciente) e também foi considerada significante na análise prévia.

Convém notar que as três primeiras variáveis (sexo, idade e doença de Chagas) são observadas para todos os indivíduos, enquanto que a fração de ejeção depende do resultado de exames, que nem sempre são realizados em todos os indivíduos. Embora 
existam vários métodos para avaliar a fração de ejeção, neste trabalho considerou-se a obtida por cintilografia. Portanto, das quatro variáveis consideradas, a fração de ejeção é a única sujeita a dados omissos, sendo que a omissão pode estar relacionada a um pior estado de saúde, dado que para que o paciente seja submetido ao exame é necessário que seu quadro clínico seja estável.

Para a comparação entre os 3 métodos (ACC e os dois tipos de ponderação), considerou-se o banco de dados com 898 pacientes como a amostra total a partir da qual foram obtidas amostras de mesmo tamanho com dados omissos simulados. Levando-se em conta a natureza das variáveis, simulou-se a omissão apenas na covariável fração de ejeção, pois era a única sujeita à omissão possivelmente relacionada a um pior prognóstico.

Foram considerados 3 cenários: baixo percentual de omissão (10\%), médio percentual de omissão (30\%) e alto percentual de omissão (50\%). Para que a omissão não fosse completamente aleatória e estivesse relacionada com a sobrevivência, foram simulados dados omissos predominantemente nas falhas e nos menores tempos observados. A idéia é que quanto maior o tempo de falha ou censura, menor a probabilidade de omissão e maior a probabilidade de cura.

Na amostra selecionada, havia 594 censuras $(66,1 \%)$ e 304 falhas $(33,9 \%)$. Primeiro os pacientes foram divididos segundo tercis de tempo, separadamente para falhas e censuras. Em seguida, foi simulada a omissão em cada subgrupo de modo que o percentual de omissão nas falhas fosse aproximadamente o dobro do percentual de omissão nas censuras (risco relativo aproximadamente igual a 2). Além disso, considerou-se que quanto menor o tempo, mais freqüente era a omissão, ou seja, o percentual de omissão era decrescente nos tercis de tempo (maior percentual no $1^{o .}$ tercil, segundo maior percentual no $2^{o .}$ tercil e menor percentual no $3^{o}$ tercil). A Tabela 4.1 mostra o percentual de omissão simulado em cada subgrupo. 
Com este esquema de simulação, o percentual de censuras também aumentou gradativamente com o aumento do percentual de omissão, sendo respectivamente $68 \%$, $74 \%$ e $83 \%$ de censuras nos cenários 10\%, 30\% e 50\% de omissão. Vale lembrar que estes são valores aproximados, uma vez que as observações com dados omissos variam a cada simulação.

Tabela 4.1: Probabilidades de omissão simuladas segundo falhas e tercis de tempo

\begin{tabular}{|c|c|c|c|c|}
\hline $\begin{array}{l}\text { Percentual } \\
\text { de omissão }\end{array}$ & Subgrupo & $\begin{array}{c}\text { Tercil } \\
\text { de tempo }\end{array}$ & $\begin{array}{l}\text { Probabilidades de } \\
\text { omissão por tercil }\end{array}$ & $\begin{array}{c}\text { Probabilidades de } \\
\text { omissão por subgrupo }\end{array}$ \\
\hline \multirow{6}{*}{ Baixa $(10 \%)$} & \multirow{3}{*}{ Falhas $(\mathrm{n}=304)$} & $1^{o}$ & 0,25 & \multirow{3}{*}{0,15} \\
\hline & & $2^{o}$ & 0,15 & \\
\hline & & $3^{o}$ & 0,05 & \\
\hline & \multirow{3}{*}{ Censuras $(\mathrm{n}=594)$} & $1^{O}$ & 0,10 & \multirow{3}{*}{0,075} \\
\hline & & $2^{o}$ & 0,08 & \\
\hline & & $3^{o}$ & 0,04 & \\
\hline \multirow{6}{*}{ Média $(30 \%)$} & & $1^{o}$ & 0,80 & \multirow{3}{*}{0,48} \\
\hline & Falhas $(\mathrm{n}=304)$ & $2^{o}$ & 0,50 & \\
\hline & & $3^{o}$ & 0,15 & \\
\hline & \multirow{3}{*}{ Censuras $(\mathrm{n}=594)$} & $1^{o}$ & 0,40 & \multirow{3}{*}{0,23} \\
\hline & & $2^{o}$ & 0,25 & \\
\hline & & $3^{o}$ & 0,05 & \\
\hline \multirow{6}{*}{ Alta $(50 \%)$} & & $1^{O}$ & 0,95 & \multirow{3}{*}{0,75} \\
\hline & Falhas $(\mathrm{n}=304)$ & $2^{o}$ & 0,75 & \\
\hline & & $3^{\circ}$ & 0,55 & \\
\hline & \multirow{3}{*}{ Censuras $(\mathrm{n}=594)$} & $1^{o}$ & 0,55 & \multirow{3}{*}{0,375} \\
\hline & & $2^{o}$ & 0,45 & \\
\hline & & $3^{o}$ & 0,12 & \\
\hline
\end{tabular}

Os modelos com fração de cura foram ajustados sob os 3 cenários (baixo, médio e alto percentual de omissão). Em cada cenário, foram realizadas 500 réplicas (500 amostras com dados omissos simulados). Os resultados a seguir são expressos em médias e desvios padrão das estimativas dos coeficientes, vício, vício relativo e erro quadrático médio. Para o cálculo do vício, considerou-se como verdadeiras as estimativas dos parâmetros com base no total de observações antes de simular a omissão (898 casos). 
Para cada parâmetro de interesse $\theta$, considere o vetor $\left(\hat{\theta}_{i}, i=1, \ldots, 500\right)$, cujo elemento $\hat{\theta}_{i}$ corresponde à estimativa obtida na $i$-ésima amostra com omissões simuladas. Seja $\hat{\theta}^{*}$ a estimativa obtida a partir da amostra total de 898 casos, isto é, as estimativas que seriam obtidas caso os dados omissos fossem observados. Com esta notação, para cada parâmetro de interesse, as quantidades apresentadas nas tabelas correspondem às expressões

$$
\begin{aligned}
\text { Média }=\sum_{i=1}^{500} \frac{\hat{\theta}_{i}}{500}, & \mathrm{DP}=\sqrt{\sum_{i=1}^{500} \frac{\left(\hat{\theta}_{i}-\text { Média }\right)^{2}}{500-1}}, \\
\text { Vício }=\text { Média }-\hat{\theta}^{*}, & \mathrm{EQM}=\mathrm{Vício}^{2}+\mathrm{DP}^{2} .
\end{aligned}
$$

A coluna estimativas sem omissão, presente nas tabelas, refere-se às estimativas pontuais e respectivos erros padrão obtidos a partir da amostra total de 898 casos, isto é, antes de simular a omissão na variável fração de ejeção.

Com base na metodologia proposta, o processo de estimação dos parâmetros foi realizado em duas etapas: uma para estimação dos pesos e outra para estimação dos parâmetros do modelo final. Os programas computacionais utilizados em cada etapa são descritos no Apêndice C.

Nos resultados descritos a seguir, o método que utiliza como pesos o recíproco das probabilidades de não omissão é referido como método de pesos logísticos e o método que pondera as observações pelo recíproco das probabilidades de cura é chamado de método de pesos FC. Conforme mencionado anteriormente, o método sem ponderação, isto é, que considera pesos iguais a 1 para todos os indivíduos, é denominado $A C C$. Em todas as análises, os modelos ponderados foram comparados ao modelo sem ponderação. Tanto no modelo Weibull quanto no modelo de Cox, adotou-se o modelo logístico para descrever a relação entre as covariáveis e as probabilidades de cura. 


\subsection{Modelos Weibull}

As estimativas dos parâmetros referentes ao termo de sobrevivência no modelo com fração de cura Weibull, considerando baixo, médio e alto percentual de omissões são apresentados, respectivamente, nas Tabelas 4.2, 4.6 e 4.10. Os vícios e erros quadráticos médios destas estimativas encontram-se nas Tabelas 4.3, 4.7 e 4.11.

Para baixa omissão (10\% das observações), com exceção da variável sexo, o método de pesos logísticos apresentou melhores resultados em relação à ACC e à ponderação FC, com estimativas, em média, menos viciadas e menor erro quadrático médio. Neste cenário, as estimativas produzidas pelo modelo sem ponderação para o parâmetro referente à variável sexo foram melhores do que as obtidas nos modelos ponderados. Em termos de vício, o pior desempenho foi do método de pesos FC. No entanto, as estimativas produzidas por este método para as variáveis sexo, Chagas e fração de ejeção apresentaram menor variabilidade que as obtidas nos outros dois métodos.

O modelo com pesos logísticos também foi melhor no cenário de médio percentual de omissões (30\% das observações). Assim como no cenário de baixa omissão, a ACC apresentou menor vício e erro quadrático médio para a variável sexo, mas para as outras três covariáveis, o método de pesos logísticos apresentou em geral estimativas menos viciadas e com menor EQM. A menor variabilidade observada para o método de pesos FC no cenário de 10\% de omissões, só ocorreu na fração de ejeção, variável em que foi simulada a omissão. Verificou-se também que, comparativamente às médias, os desvios padrão das estimativas referentes às variáveis sexo e idade obtidas pelos três métodos foram muito altos. 
Na situação em que metade das observações apresentam dados omissos (alta omissão), as estimativas das amostras simuladas ficaram muito distantes das verdadeiras (obtidas a partir dos dados sem omissão), principalmente no que se refere às variáveis categorizadas sexo e doença de Chagas e para o parâmetro de escala. Neste cenário, a ponderação, seja por pesos logísticos ou por pesos FC, não trouxe benefícios e até comprometeu a estimação dos parâmetros.

Nos três cenários, as estimativas referentes ao termo de cura apresentaram muita instabilidade, alta imprecisão e viés em relação às estimativas com todas as observações (Tabelas 4.5, 4.9 e 4.13). Esta discrepância não foi observada nos modelos semiparamétricos apresentados na seção 4.2. É importante ressaltar que, mesmo no modelo com os 898 casos, os erros padrão das estimativas foram consideravelmente elevados, principalmente nas variáveis categorizadas e intercepto. No cenário de $50 \%$ de omissões, o método de pesos FC superou os outros dois métodos nas estimativas referentes às variáveis quantitativas (idade e fração de ejeção). Nos cenários de baixa e média omissão (10\% e 30\%), o método de pesos logísticos apresentou, em média, estimativas menos viciadas para a variável fração de ejeção.

Convém notar que a estimação dos modelos Weibull com fração de cura apresentou algumas dificuldades computacionais no que se refere à taxa de convergência. Observou-se que, em muitas amostras, o número de iterações excedeu o número máximo estabelecido que já era alto (100 iterações). Isto ocorreu mais frequentemente na ponderação pelas probabilidades de cura e no cenário de baixo percentual de omissões. O percentual de resultados que não convergiram nos cenários de baixa, média e alta omissão foi respectivamente, $6 \%, 13 \%$ e $17 \%$ do total de simulações. 
Tabela 4.2: Estimativas dos parâmetros do termo de sobrevivência para o modelo Weibull Baixo percentual de omissão (10\%)

\begin{tabular}{lcccc}
\hline Variável & $\begin{array}{c}\text { Total } \\
\text { média }(\mathrm{dp})\end{array}$ & $\begin{array}{c}\text { ACC } \\
\text { média }(\mathrm{dp})\end{array}$ & $\begin{array}{c}\text { Pesos Logísticos } \\
\text { média }(\mathrm{dp})\end{array}$ & $\begin{array}{c}\text { Pesos FC } \\
\text { média }(\mathrm{dp})\end{array}$ \\
\hline Sexo & $0,3476(0,2475)$ & $0,3315(0,1216)$ & $0,3099(0,1185)$ & $0,2473(0,1000)$ \\
Idade & $-0,0087(0,0085)$ & $-0,0072(0,0057)$ & $-0,0090(0,0046)$ & $-0,0034(0,0066)$ \\
Chagas & $0,9817(0,2333)$ & $0,9841(0,1009)$ & $0,9700(0,1021)$ & $1,0265(0,0882)$ \\
Feve & $-0,0384(0,0142)$ & $-0,0410(0,0060)$ & $-0,0380(0,0062)$ & $-0,0457(0,0059)$ \\
Intercepto & $-3,1624(0,5366)$ & $-3,2583(0,3400)$ & $-3,1217(0,2889)$ & $-3,3510(0,3735)$ \\
$\log (\delta)$ & $0,0990(0,0579)$ & $0,1383(0,0393)$ & $0,1061(0,0072)$ & $0,1276(0,0286)$ \\
\hline
\end{tabular}

Tabela 4.3: Vícios e erros quadráticos médios das estimativas dos parâmetros do termo de sobrevivência para o modelo Weibull - Baixo percentual de omissão (10\%)

\begin{tabular}{lcccccc}
\hline & \multicolumn{3}{c}{ Vício } & \multicolumn{3}{c}{ EQM } \\
\hline Variável & ACC & Logísticos & FC & ACC & Logísticos & FC \\
\hline Sexo & $-0,0161$ & $-0,0377$ & $-0,1003$ & 0,0150 & 0,0155 & 0,0201 \\
Idade & 0,0015 & $-0,0003$ & 0,0053 & $3,45 \times 10^{-5}$ & $2,17 \times 10^{-5}$ & $7,13 \times 10^{-5}$ \\
Chagas & 0,0024 & $-0,0118$ & 0,0448 & 0,0102 & 0,0106 & 0,0098 \\
Feve & $-0,0027$ & 0,0004 & $-0,0074$ & $4,32 \times 10^{-5}$ & $3,86 \times 10^{-5}$ & $8,89 \times 10^{-5}$ \\
Intercepto & $-0,0959$ & 0,0407 & $-0,1886$ & 0,1248 & 0,0851 & 0,1750 \\
$\log (\delta)$ & 0,0474 & 0,0099 & 0,0335 & 0,0025 & 0,0011 & 0,0020 \\
\hline
\end{tabular}

Tabela 4.4: Estimativas dos parâmetros do termo de cura para o modelo Weibull - Baixo percentual de omissão (10\%)

\begin{tabular}{lcccc}
\hline Variável & Total & ACC & Pesos Logísticos & Pesos FC \\
& média $(\mathrm{dp})$ & média $(\mathrm{dp})$ & média $(\mathrm{dp})$ & média $(\mathrm{dp})$ \\
\hline Sexo & $-0,4757(0,8937)$ & $-0,8655(4,4171)$ & $-0,3227(5,1536)$ & $-1,9904(4,3538)$ \\
Idade & $0,0524(0,0259)$ & $0,0544(0,0986)$ & $0,0649(0,1332)$ & $0,0721(0,0164)$ \\
Chagas & $-0,0842(0,8452)$ & $-0,8374(2,4974)$ & $-0,3279(2,5340)$ & $-2,4811(3,3585)$ \\
Feve & $-0,0494(0,0335)$ & $-0,0087(0,6524)$ & $-0,0417(0,1003)$ & $0,0293(0,0976)$ \\
Intercepto & $0,4486(1,4707)$ & $1,4093(6,1212)$ & $0,9321(7,2870)$ & $2,5728(6,4950)$ \\
\hline
\end{tabular}


Tabela 4.5: Vícios e erros quadráticos médios das estimativas dos parâmetros do termo de cura para o modelo Weibull - Baixo percentual de omissão (10\%)

\begin{tabular}{lcccccc}
\hline & \multicolumn{3}{c}{ Vício } & \multicolumn{3}{c}{ EQM } \\
\hline Variável & ACC & Logísticos & FC & ACC & Logísticos & FC \\
\hline Sexo & $-0,8179$ & $-0,2752$ & $-1,9428$ & 20,1793 & 26,6357 & 22,7299 \\
Idade & 0,0020 & 0,0125 & 0,0197 & $9,73 \times 10^{-3}$ & $1,79 \times 10^{-2}$ & $6,56 \times 10^{-4}$ \\
Chagas & $-0,7532$ & $-0,2437$ & $-2,3969$ & 6,8043 & 6,4804 & 17,0243 \\
Feve & 0,0408 & 0,0077 & 0,0787 & 0,4272 & 0,0101 & 0,0157 \\
Intercepto & 0,9607 & 0,4835 & 2,1242 & 38,3919 & 53,3349 & 46,6967 \\
\hline
\end{tabular}

Tabela 4.6: Estimativas dos parâmetros do termo de sobrevivência para o modelo Weibull Médio percentual de omissão $(30 \%)$

\begin{tabular}{lcccc}
\hline Variável & Total & ACC & Pesos Logísticos & Pesos FC \\
& média $(\mathrm{dp})$ & média $(\mathrm{dp})$ & média $(\mathrm{dp})$ & média $(\mathrm{dp})$ \\
\hline Sexo & $0,3476(0,2475)$ & $0,2071(0,2150)$ & $0,1923(0,2253)$ & $0,0741(0,2158)$ \\
Idade & $-0,0087(0,0085)$ & $-0,0054(0,0083)$ & $-0,0086(0,0089)$ & $-0,0041(0,0119)$ \\
Chagas & $0,9817(0,2333)$ & $0,6985(0,2162)$ & $0,8001(0,2317)$ & $0,6554(0,2637)$ \\
Feve & $-0,0384(0,0142)$ & $-0,0409(0,0084)$ & $-0,0361(0,0133)$ & $-0,0402(0,0087)$ \\
Intercepto & $-3,1624(0,5366)$ & $-3,3046(0,5106)$ & $-3,0041(0,5047)$ & $-3,2273(0,6019)$ \\
$\log (\delta)$ & $0,0990(0,0579)$ & $0,4072(0,0813)$ & $0,2080(0,0942)$ & $0,4428(0,1064)$ \\
\hline
\end{tabular}

Tabela 4.7: Vícios e erros quadráticos médios das estimativas dos parâmetros do termo de sobrevivência para o modelo Weibull - Médio percentual de omissão (30\%)

\begin{tabular}{lcccccc}
\hline & \multicolumn{3}{c}{ Vício } & \multicolumn{3}{c}{ EQM } \\
\hline Variável & ACC & Logísticos & FC & ACC & Logísticos & FC \\
\hline Sexo & $-0,1405$ & $-0,1553$ & $-0,2735$ & 0,0660 & 0,0749 & 0,1214 \\
Idade & 0,0033 & 0,0001 & 0,0046 & $7,95 \times 10^{-5}$ & $7,84 \times 10^{-5}$ & $1,62 \times 10^{-4}$ \\
Chagas & $-0,2832$ & $-0,1817$ & $-0,3263$ & 0,1269 & 0,0867 & 0,1760 \\
Feve & $-0,0026$ & 0,0022 & $-0,0018$ & $7,79 \times 10^{-5}$ & $1,82 \times 10^{-4}$ & $7,92 \times 10^{-5}$ \\
Intercepto & $-0,1422$ & 0,1583 & $-0,0649$ & 0,2810 & 0,2798 & 0,3665 \\
$\log (\delta)$ & 0,3082 & 0,1091 & 0,3438 & 0,1016 & 0,0208 & 0,1295 \\
\hline
\end{tabular}


Tabela 4.8: Estimativas dos parâmetros do termo de cura para o modelo Weibull - Médio percentual de omissão (30\%)

\begin{tabular}{lcccc}
\hline Variável & $\begin{array}{c}\text { Total } \\
\text { média }(\mathrm{dp})\end{array}$ & $\begin{array}{c}\text { ACC } \\
\text { média }(\mathrm{dp})\end{array}$ & $\begin{array}{c}\text { Pesos Logísticos } \\
\text { média }(\mathrm{dp})\end{array}$ & $\begin{array}{c}\text { Pesos FC } \\
\text { média }(\mathrm{dp})\end{array}$ \\
\hline Sexo & $-0,4757(0,8937)$ & $-3,7710(15,3521)$ & $-0,6984(8,3763)$ & $0,1417(2,8743)$ \\
Idade & $0,0524(0,0259)$ & $0,1132(0,4641)$ & $0,2019(1,1335)$ & $0,2709(1,2544)$ \\
Chagas & $-0,0842(0,8452)$ & $-3,3436(19,7486)$ & $-1,6889(16,4293)$ & $0,7044(3,6385)$ \\
Feve & $-0,0494(0,0335)$ & $0,0711(0,9810)$ & $-0,0662(0,9999)$ & $0,0914(0,9517)$ \\
Intercepto & $0,4486(1,4707)$ & $3,2473(21,7068)$ & $0,7693(6,4154)$ & $-0,3555(3,8234)$ \\
\hline
\end{tabular}

Tabela 4.9: Vícios e erros quadráticos médios das estimativas dos parâmetros do termo de cura para o modelo Weibull - Médio percentual de omissão (30\%)

\begin{tabular}{lcccccc}
\hline & \multicolumn{3}{c}{ Vício } & & \multicolumn{3}{c}{ EQM } \\
\hline Variável & ACC & Logísticos & FC & ACC & Logísticos & FC \\
\hline Sexo & $-3,7234$ & $-0,6508$ & 0,1892 & 249,5494 & 70,5854 & 8,2972 \\
Idade & 0,0608 & 0,1495 & 0,2185 & 0,2191 & 1,3073 & 1,6213 \\
Chagas & $-3,2593$ & $-1,6046$ & 0,7886 & 400,6317 & 272,4956 & 13,8603 \\
Feve & 0,1206 & $-0,0168$ & 0,1409 & 0,9768 & 1,0000 & 0,9255 \\
Intercepto & 2,7987 & 0,3207 & $-0,8041$ & 479,0194 & 41,2604 & 15,2649 \\
\hline
\end{tabular}

Tabela 4.10: Estimativas dos parâmetros do termo de sobrevivência para o modelo Weibull - Alto percentual de omissão (50\%)

\begin{tabular}{lcccc}
\hline Variável & $\begin{array}{c}\text { Total } \\
\text { média }(\mathrm{dp})\end{array}$ & $\begin{array}{c}\text { ACC } \\
\text { média }(\mathrm{dp})\end{array}$ & $\begin{array}{c}\text { Pesos Logísticos } \\
\text { média }(\mathrm{dp})\end{array}$ & $\begin{array}{c}\text { Pesos FC } \\
\text { média }(\mathrm{dp})\end{array}$ \\
\hline Sexo & $0,3476(0,2475)$ & $0,0466(0,4171)$ & $0,0612(0,2796)$ & $-0,0351(0,4155)$ \\
Idade & $-0,0087(0,0085)$ & $-0,0092(0,0166)$ & $-0,0066(0,0116)$ & $0,0020(0,0225)$ \\
Chagas & $0,9817(0,2333)$ & $0,5480(0,3909)$ & $0,4879(0,2630)$ & $0,4010(0,4217)$ \\
Feve & $-0,0384(0,0142)$ & $-0,0367(0,0168)$ & $-0,0285(0,0162)$ & $-0,0355(0,0165)$ \\
Intercepto & $-3,1624(0,5366)$ & $-3,0150(0,9850)$ & $-2,9286(0,6933)$ & $-3,4338(1,1810)$ \\
$\log (\delta)$ & $0,0990(0,0579)$ & $0,6088(0,1367)$ & $0,5143(0,1436)$ & $0,6608(0,1711)$ \\
\hline
\end{tabular}


Tabela 4.11: Vícios e erros quadráticos médios das estimativas dos parâmetros do termo de sobrevivência para o modelo Weibull - Alto percentual de omissão (50\%)

\begin{tabular}{lcccccc}
\hline & \multicolumn{3}{c}{ Vício } & \multicolumn{3}{c}{ EQM } \\
\hline Variável & ACC & Logísticos & FC & ACC & Logísticos & FC \\
\hline Sexo & $-0,3010$ & $-0,2864$ & $-0,3827$ & 0,2646 & 0,1602 & 0,3191 \\
Idade & $-0,0005$ & 0,0021 & 0,0108 & $2,74 \times 10^{-4}$ & $1,39 \times 10^{-4}$ & $6,23 \times 10^{-4}$ \\
Chagas & $-0,4337$ & $-0,4938$ & $-0,5807$ & 0,3410 & 0,3130 & 0,5151 \\
Feve & 0,0016 & 0,0098 & 0,0029 & $2,86 \times 10^{-4}$ & $3,60 \times 10^{-4}$ & $2,80 \times 10^{-4}$ \\
Intercepto & 0,1474 & 0,2338 & $-0,2715$ & 0,9919 & 0,5353 & 1,4685 \\
$\log (\delta)$ & 0,5098 & 0,4153 & 0,5618 & 0,2786 & 0,1931 & 0,3449 \\
\hline
\end{tabular}

Tabela 4.12: Estimativas dos parâmetros do termo de cura para o modelo Weibull - Alto percentual de omissão (50\%)

\begin{tabular}{lcccc}
\hline Variável & Total & ACC & Pesos Logísticos & Pesos FC \\
& média $(\mathrm{dp})$ & média $(\mathrm{dp})$ & média $(\mathrm{dp})$ & média $(\mathrm{dp})$ \\
\hline Sexo & $-0,4757(0,8937)$ & $-3,7577(20,5569)$ & $0,0392(18,0631)$ & $-0,8583(10,4179)$ \\
Idade & $0,0524(0,0259)$ & $0,2861(1,5494)$ & $0,3372(1,6780)$ & $0,1322(1,3768)$ \\
Chagas & $-0,0842(0,8452)$ & $-0,6191(18,7618)$ & $-0,4400(24,8730)$ & $7,0828(44,4319)$ \\
Feve & $-0,0494(0,0335)$ & $-0,0512(1,7590)$ & $-0,0628(1,4986)$ & $-0,0503(1,3415)$ \\
Intercepto & $0,4486(1,4707)$ & $0,6770(23,6385)$ & $-2,1552(32,2872)$ & $4,1766(38,5447)$ \\
\hline
\end{tabular}

Tabela 4.13: Vícios e erros quadráticos médios das estimativas dos parâmetros do termo de cura para o modelo Weibull - Alto percentual de omissão (50\%)

\begin{tabular}{lcccccc}
\hline & \multicolumn{3}{c}{ Vício } & \multicolumn{3}{c}{ EQM } \\
\hline Variável & ACC & Logísticos & FC & ACC & Logísticos & FC \\
\hline Sexo & $-3,7101$ & 0,0868 & $-0,8107$ & $4,36 \times 10^{2}$ & $3,26 \times 10^{2}$ & $1,09 \times 10^{2}$ \\
Idade & 0,2337 & 0,2848 & 0,0798 & 2,4552 & 2,8969 & 1,9019 \\
Chagas & $-0,5348$ & $-0,3558$ & 7,1671 & $3,52 \times 10^{2}$ & $6,19 \times 10^{2}$ & $2,03 \times 10^{3}$ \\
Feve & $-0,0017$ & $-0,0134$ & $-0,0009$ & 3,0942 & 2,2461 & 1,7996 \\
Intercepto & 0,2283 & $-2,6038$ & 3,7280 & $5,59 \times 10^{2}$ & $1,05 \times 10^{3}$ & $1,50 \times 10^{3}$ \\
\hline
\end{tabular}




\subsection{Modelos semiparamétricos}

Nesta seção são apresentados os resultados dos modelos com fração de cura semiparamétricos. Assim como no modelo paramétrico, os resultados em cada cenário estão divididos em duas tabelas: uma para as estimativas relacionadas aos tempos de sobrevivência e outra para as estimativas relacionadas à probabilidade de cura.

Convém lembrar que, nos modelos considerados, o que se avalia é a influência das covariáveis sobre a taxa de falha, no caso do modelo de Cox, e sobre a chance de não cura, no caso do modelo logístico. Portanto, coeficientes positivos indicam aumento do risco de óbito (ou da chance de ser não curado) e coeficientes negativos indicam redução do risco de óbito (ou da chance de não cura). Neste contexto, sob o ponto de vista clínico, espera-se valores positivos para os fatores de risco (sexo masculino, idade e doença de Chagas) e valores negativos para a fração de ejeção.

Os erros padrão das estimativas dos coeficientes dos modelos semiparamétricos com base nos dados completos foram estimados pelo método bootstrap não paramétrico (Efron (1982)). Foram sorteadas 500 amostras de mesmo tamanho (898 casos) e os erros padrão correspondem aos desvios padrão das 500 estimativas obtidas.

\subsubsection{Baixo percentual de omissão}

As Tabelas 4.14 a 4.17 apresentam os resultados produzidos pelos três métodos (ACC, pesos logísticos e pesos FC), considerando omissão em 10\% das observações.

Tanto no termo de sobrevivência quanto no termo de cura, o método dos pesos logísticos foi o que apresentou melhor desempenho, produzindo estimativas menos viciadas e com menor erro quadrático médio para todas as covariáveis analisadas. 
No termo de sobrevivência, as estimativas produzidas pelo método da ponderação pela fração de cura (FC) foram menos precisas e apresentaram maior vício que os outros dois métodos. Este comportamento também foi observado no termo de cura para as variáveis sexo, idade e doença de Chagas. Para a variável fração de ejeção em que foi simulada a omissão, a variabilidade das estimativas obtidas pelo método FC foi menor e o vício foi praticamente o mesmo da ACC.

Neste cenário de baixo percentual de omissão, as estimativas produzidas pelo método de pesos logísticos foram muito próximas às estimativas na $\mathrm{ACC}$. O método de pesos FC foi o que mais se diferenciou, sendo que as maiores discrepâncias foram observadas nos parâmetros relacionados à taxa de cura. Para a variável Chagas, o coeficiente estimado chegou a ser maior que o dobro dos valores produzidos pelos outros dois métodos, com vício relativo superior a 100\%. Em termos de estimativas de risco relativo, o valor esperado caso os dados omissos tivessem sido observados é de aproximadamente 2,8 , o que significa que espera-se que pacientes chagásicos tenham quase 3 vezes mais chance de serem não curados do que pacientes não chagásicos. Para o método FC, a estimativa de risco relativo foi superior a 8, atribuindo uma probabilidade de não cura muito maior que a esperada para os pacientes chagásicos.

Com o intuito de explicar o pior desempenho do método de pesos FC, investigou-se mais detalhadamente o processo de estimação dos pesos para este método. Analisando as amostras simuladas, observou-se que, em média, as probabilidades estimadas de cura eram muito baixas. Em uma das amostras, por exemplo, o maior valor estimado foi 0,12 . Isto aumenta consideravelmente a magnitude dos pesos definidos pelo recíproco das probabilidades de cura. Observou-se também que, nos modelos para estimar as probabilidades de cura, a inclusão da variável explicativa indicadora de omissão (com valores iguais a 1 em apenas 10\% da amostra) reduzia muito a precisão das estimativas, o que naturalmente prejudica a estimação dos pesos FC. 
Tabela 4.14: Estimativas dos parâmetros do termo de sobrevivência para o modelo semiparamétrico - Baixo percentual de omissão (10\%)

\begin{tabular}{lcccc}
\hline Variável & $\begin{array}{c}\text { Total } \\
\text { média }(\mathrm{dp})\end{array}$ & $\begin{array}{c}\text { ACC } \\
\text { média }(\mathrm{dp})\end{array}$ & $\begin{array}{c}\text { Pesos Logísticos } \\
\text { média }(\mathrm{dp})\end{array}$ & $\begin{array}{c}\text { Pesos FC } \\
\text { média }(\mathrm{dp})\end{array}$ \\
\hline Sexo & $0,2217(0,1492)$ & $0,2101(0,0509)$ & $0,2130(0,0491)$ & $0,1701(0,0600)$ \\
Idade & $0,0060(0,0055)$ & $0,0068(0,0022)$ & $0,0058(0,0021)$ & $0,0085(0,0029)$ \\
Chagas & $0,8256(0,1325)$ & $0,8129(0,0526)$ & $0,8256(0,0476)$ & $0,8101(0,0547)$ \\
Feve & $-0,0445(0,0087)$ & $-0,0462(0,0031)$ & $-0,0448(0,0033)$ & $-0,0395(0,0037)$ \\
\hline
\end{tabular}

Tabela 4.15: Vícios e erros quadráticos médios das estimativas dos parâmetros do termo de sobrevivência para o modelo semiparamétrico - Baixo percentual de omissão (10\%)

\begin{tabular}{lcccccc}
\hline & \multicolumn{3}{c}{ Vício } & \multicolumn{3}{c}{ EQM } \\
\hline Variável & ACC & Logísticos & FC & ACC & Logísticos & FC \\
\hline Sexo & $-0,0115$ & $-0,0086$ & $-0,0515$ & 0,0027 & 0,0025 & 0,0063 \\
Idade & 0,0008 & $-0,0001$ & 0,0025 & $5,74 \times 10^{-6}$ & $4,37 \times 10^{-6}$ & $1,48 \times 10^{-5}$ \\
Chagas & $-0,0127$ & 0,0000 & $-0,0155$ & 0,0029 & 0,0023 & 0,0032 \\
Feve & $-0,0017$ & $-0,0003$ & 0,0050 & $1,26 \times 10^{-5}$ & $1,07 \times 10^{-5}$ & $3,84 \times 10^{-5}$ \\
\hline
\end{tabular}

Tabela 4.16: Estimativas dos parâmetros do termo de cura para o modelo semiparamétrico Baixo percentual de omissão (10\%)

\begin{tabular}{lcccc}
\hline Variável & $\begin{array}{c}\text { Total } \\
\text { média }(\mathrm{dp})\end{array}$ & $\begin{array}{c}\text { ACC } \\
\text { média }(\mathrm{dp})\end{array}$ & $\begin{array}{c}\text { Pesos Logísticos } \\
\text { média }(\mathrm{dp})\end{array}$ & $\begin{array}{c}\text { Pesos FC } \\
\text { média }(\mathrm{dp})\end{array}$ \\
\hline Sexo & $1,0552(0,3947)$ & $1,0621(0,1779)$ & $1,0548(0,1794)$ & $1,3721(0,1901)$ \\
Idade & $0,0175(0,0184)$ & $0,0181(0,0076)$ & $0,0179(0,0076)$ & $0,0073(0,0131)$ \\
Chagas & $1,0388(0,6996)$ & $1,0158(0,1544)$ & $1,0179(0,1495)$ & $2,1324(0,2332)$ \\
Feve & $-0,1067(0,0187)$ & $-0,1085(0,0119)$ & $-0,1082(0,0121)$ & $-0,1087(0,0088)$ \\
\hline
\end{tabular}


Tabela 4.17: Vícios e erros quadráticos médios das estimativas dos parâmetros do termo de cura para o modelo semiparamétrico - Baixo percentual de omissão (10\%)

\begin{tabular}{lcccccc}
\hline & \multicolumn{3}{c}{ Vício } & \multicolumn{3}{c}{ EQM } \\
\hline Variável & ACC & Logísticos & FC & ACC & Logísticos & FC \\
\hline Sexo & 0,0069 & $-0,0003$ & 0,3169 & 0,0317 & 0,0322 & 0,1366 \\
Idade & 0,0006 & 0,0004 & $-0,0102$ & $5,82 \times 10^{-5}$ & $5,79 \times 10^{-5}$ & $2,76 \times 10^{-4}$ \\
Chagas & $-0,0230$ & $-0,0209$ & 1,0935 & 0,0244 & 0,0228 & 1,2502 \\
Feve & $-0,0018$ & $-0,0014$ & $-0,0019$ & $1,44 \times 10^{-4}$ & $1,47 \times 10^{-4}$ & $8,15 \times 10^{-5}$ \\
Intercepto & $-0,1448$ & $-0,0139$ & $-0,0018$ & 0,3187 & 0,2932 & 0,6011 \\
\hline
\end{tabular}

\subsubsection{Médio percentual de omissão}

As Tabelas 4.18 a 4.21 apresentam, respectivamente, os resultados do termo de sobrevivência e do termo de cura do modelo com fração de cura semiparamétrico, considerando omissão em $30 \%$ das observações.

Assim como no cenário de baixo percentual de omissão, nos parâmetros relacionados à sobrevivência, os resultados foram melhores para o modelo com pesos logísticos. Este método produziu, em média, estimativas bem menos viciadas e menor erro quadrático médio para todas as covariáveis. Convém notar que as estimativas obtidas pelo método de pesos logísticos, apesar de menos viciadas, apresentaram maiores desvios padrão. Neste termo de sobrevivência, o método de pesos FC apresentou maior vício e maior erro quadrático médio nas covariáveis sexo e idade. No entanto, para Chagas e fração de ejeção, seu desempenho foi ligeiramente melhor que a ACC.

Nos parâmetros referentes à fração de cura, o método de pesos logísticos superou os outros dois métodos apenas nas variáveis idade e Chagas. Na variável sexo, a ponderação não beneficiou a estimação do parâmetro, sendo que as estimativas obtidas com a ACC foram menos viciadas e mais precisas. A ACC também apresentou menor vício para a variável fração de ejeção, em que foi simulada a omissão. Nesta variável, 
a variabilidade das estimativas produzidas pelo método de pesos FC foi consideravelmente menor que na ACC e na ponderação logística. Esta estabilidade também foi observada no cenário de $10 \%$ de omissão.

Assim como na análise anterior, observou-se que, no modelo para cura, o método de pesos FC tende a subestimar o coeficiente referente à idade e tende a superestimar os coeficientes das variáveis categorizadas sexo e doença de Chagas.

Tabela 4.18: Estimativas dos parâmetros do termo de sobrevivência para o modelo semiparamétrico - Médio percentual de omissão (30\%)

\begin{tabular}{lcccc}
\hline Variável & $\begin{array}{c}\text { Total } \\
\text { média }(\mathrm{dp})\end{array}$ & $\begin{array}{c}\text { ACC } \\
\text { média }(\mathrm{dp})\end{array}$ & $\begin{array}{c}\text { Pesos Logísticos } \\
\text { média }(\mathrm{dp})\end{array}$ & $\begin{array}{c}\text { Pesos FC } \\
\text { média }(\mathrm{dp})\end{array}$ \\
\hline Sexo & $0,2217(0,1492)$ & $0,1455(0,0889)$ & $0,1947(0,1122)$ & $0,1060(0,0891)$ \\
Idade & $0,0060(0,0055)$ & $0,0098(0,0047)$ & $0,0058(0,0056)$ & $0,0111(0,0061)$ \\
Chagas & $0,8256(0,1325)$ & $0,6688(0,1181)$ & $0,8040(0,1383)$ & $0,6997(0,1339)$ \\
Feve & $-0,0445(0,0087)$ & $-0,0523(0,0068)$ & $-0,0463(0,0086)$ & $-0,0501(0,0076)$ \\
\hline
\end{tabular}

Tabela 4.19: Vícios e erros quadráticos médios das estimativas dos parâmetros do termo de sobrevivência para o modelo semiparamétrico - Médio percentual de omissão (30\%)

\begin{tabular}{lcccccc}
\hline & \multicolumn{3}{c}{ Vício } & \multicolumn{3}{c}{ EQM } \\
\hline Variável & ACC & Logísticos & FC & ACC & Logísticos & FC \\
\hline Sexo & $-0,0761$ & $-0,0270$ & $-0,1157$ & 0,0137 & 0,0133 & 0,0213 \\
Idade & 0,0038 & $-0,0002$ & 0,0051 & $3,72 \times 10^{-5}$ & $3,13 \times 10^{-5}$ & $6,34 \times 10^{-4}$ \\
Chagas & $-0,1568$ & $-0,0216$ & $-0,1260$ & 0,0386 & 0,0196 & 0,0338 \\
Feve & $-0,0079$ & $-0,0018$ & $-0,0057$ & $1,08 \times 10^{-4}$ & $7,66 \times 10^{-5}$ & $8,96 \times 10^{-5}$ \\
\hline
\end{tabular}


Tabela 4.20: Estimativas dos parâmetros do termo de cura para o modelo semiparamétrico Médio percentual de omissão (30\%)

\begin{tabular}{lcccc}
\hline Variável & $\begin{array}{c}\text { Total } \\
\text { média }(\mathrm{dp})\end{array}$ & $\begin{array}{c}\text { ACC } \\
\text { média }(\mathrm{dp})\end{array}$ & $\begin{array}{c}\text { Pesos Logísticos } \\
\text { média }(\mathrm{dp})\end{array}$ & $\begin{array}{c}\text { Pesos FC } \\
\text { média }(\mathrm{dp})\end{array}$ \\
\hline Sexo & $1,0552(0,3947)$ & $1,0890(0,2897)$ & $1,0958(0,3319)$ & $1,1963(0,3361)$ \\
Idade & $0,0175(0,0184)$ & $0,0217(0,0148)$ & $0,0216(0,0146)$ & $0,0148(0,0272)$ \\
Chagas & $1,0388(0,6996)$ & $1,0007(0,3368)$ & $1,0327(0,3807)$ & $1,6245(0,2936)$ \\
Feve & $-0,1067(0,0187)$ & $-0,1069(0,0210)$ & $-0,1054(0,0252)$ & $-0,1000(0,0141)$ \\
Intercepto & $4,5652(0,9965)$ & $3,5013(1,0762)$ & $4,0209(1,1501)$ & $3,4784(1,5177)$ \\
\hline
\end{tabular}

Tabela 4.21: Vícios e erros quadráticos médios das estimativas dos parâmetros do termo de cura para o modelo semiparamétrico - Médio percentual de omissão (30\%)

\begin{tabular}{lcccccc}
\hline & \multicolumn{3}{c}{ Vício } & \multicolumn{3}{c}{ EQM } \\
\hline Variável & ACC & Logísticos & FC & ACC & Logísticos & FC \\
\hline Sexo & 0,0339 & 0,0406 & 0,1411 & 0,0850 & 0,1118 & 0,1329 \\
Idade & 0,0042 & 0,0041 & $-0,0027$ & $2,36 \times 10^{-4}$ & $2,31 \times 10^{-4}$ & $7,47 \times 10^{-4}$ \\
Chagas & $-0,0381$ & $-0,0061$ & 0,5857 & 0,1149 & 0,1449 & 0,4293 \\
Feve & $-0,0002$ & 0,0014 & 0,0067 & $4,42 \times 10^{-4}$ & $6,35 \times 10^{-4}$ & $2,43 \times 10^{-4}$ \\
Intercepto & $-1,0639$ & $-0,5444$ & $-1,0868$ & 2,2901 & 1,6191 & 3,4847 \\
\hline
\end{tabular}

\subsubsection{Alto percentual de omissão}

As Tabelas 4.22 a 4.25 apresentam, respectivamente, os resultados do termo de sobrevivência e do termo de cura, considerando omissão em 50\% das observações.

Neste cenário, em todos os parâmetros do modelo de sobrevivência, o método de pesos logísticos produziu estimativas, em média, menos viciadas que os outros dois métodos. Neste método, houve mais variabilidade das estimativas relacionadas à fração de ejeção, o que resultou em um erro quadrático médio um pouco maior para esta variável. As estimativas obtidas pelo método de pesos FC para os parâmetros referentes 
a sexo e idade foram, em média, mais viciadas que nos outros dois métodos. Além disso, com exceção da fração de ejeção, observou-se maior variabilidade das estimativas produzidas pelo método FC. Com relação ao vício, a ponderação FC superou a ACC apenas na variável Chagas.

Nos parâmetros relacionados à probabilidade de cura, para a variável fração de ejeção em que foi simulada a omissão, o método de pesos FC superou os outros dois métodos, apresentando menor vício e menor erro quadrático médio. Este método também apresentou estimativas, em média, menos viciadas para o parâmetro referente à idade. Neste termo do modelo, a ponderação, tanto logística quanto FC, não melhorou as estimativas para as variáveis categorizadas sexo e doença de Chagas.

Apesas do método $\mathrm{FC}$ continuar superestimando as estimativas de risco de não cura para os chagásicos, as diferenças em relação à ACC e ponderação logística foram consideravelmente menores que as observadas nos cenários anteriores.

Uma análise comparativa entre os três cenários é apresentada na seção 4.2.4.

Tabela 4.22: Estimativas dos parâmetros do termo de sobrevivência para o modelo semiparamétrico - Alto percentual de omissão (50\%)

\begin{tabular}{lcccc}
\hline Variável & $\begin{array}{c}\text { Total } \\
\text { média }(\mathrm{dp})\end{array}$ & $\begin{array}{c}\text { ACC } \\
\text { média }(\mathrm{dp})\end{array}$ & $\begin{array}{c}\text { Pesos Logísticos } \\
\text { média }(\mathrm{dp})\end{array}$ & $\begin{array}{c}\text { Pesos FC } \\
\text { média }(\mathrm{dp})\end{array}$ \\
\hline Sexo & $0,2217(0,1492)$ & $0,0220(0,2024)$ & $0,1279(0,1998)$ & $-0,0262(0,2448)$ \\
Idade & $0,0060(0,0055)$ & $0,0104(0,0100)$ & $0,0067(0,0092)$ & $0,0118(0,0115)$ \\
Chagas & $0,8256(0,1325)$ & $0,5937(0,2560)$ & $0,7261(0,2264)$ & $0,6493(0,2756)$ \\
Feve & $-0,0445(0,0087)$ & $-0,0502(0,0157)$ & $-0,0497(0,0165)$ & $-0,0502(0,0160)$ \\
\hline
\end{tabular}


Tabela 4.23: Vícios e erros quadráticos médios das estimativas dos parâmetros do termo de sobrevivência para o modelo semiparamétrico - Alto percentual de omissão (50\%)

\begin{tabular}{lcccccc}
\hline & \multicolumn{3}{c}{ Vício } & \multicolumn{3}{c}{ EQM } \\
\hline Variável & ACC & Logísticos & FC & ACC & Logísticos & FC \\
\hline Sexo & $-0,1997$ & $-0,0937$ & $-0,2479$ & 0,0809 & 0,0487 & 0,1213 \\
Idade & 0,0044 & 0,0007 & 0,0058 & $1,19 \times 10^{-4}$ & $8,44 \times 10^{-5}$ & $1,65 \times 10^{-4}$ \\
Chagas & $-0,2320$ & $-0,0995$ & $-0,1763$ & 0,1193 & 0,0612 & 0,1070 \\
Feve & $-0,0058$ & $-0,0052$ & $-0,0057$ & $2,80 \times 10^{-4}$ & $2,99 \times 10^{-4}$ & $2,89 \times 10^{-4}$ \\
\hline
\end{tabular}

Tabela 4.24: Estimativas dos parâmetros do termo de cura para o modelo semiparamétrico Alto percentual de omissão (50\%)

\begin{tabular}{lcccc}
\hline Variável & $\begin{array}{c}\text { Total } \\
\text { média }(\mathrm{dp})\end{array}$ & $\begin{array}{c}\text { ACC } \\
\text { média }(\mathrm{dp})\end{array}$ & $\begin{array}{c}\text { Pesos Logísticos } \\
\text { média }(\mathrm{dp})\end{array}$ & $\begin{array}{c}\text { Pesos FC } \\
\text { média }(\mathrm{dp})\end{array}$ \\
\hline Sexo & $1,0552(0,3947)$ & $1,0645(0,5052)$ & $1,1205(0,5662)$ & $1,0181(0,5911)$ \\
Idade & $0,0175(0,0184)$ & $0,0207(0,0280)$ & $0,0254(0,0268)$ & $0,0164(0,0417)$ \\
Chagas & $1,0388(0,6996)$ & $1,4405(0,7090)$ & $1,4866(0,7496)$ & $1,6828(0,9777)$ \\
Feve & $-0,1067(0,0187)$ & $-0,1268(0,0408)$ & $-0,1364(0,0588)$ & $-0,1150(0,0389)$ \\
Intercepto & $4,5652(0,9965)$ & $2,8175(1,9568)$ & $4,1438(2,1866)$ & $2,7203(2,4943)$ \\
\hline
\end{tabular}

Tabela 4.25: Vícios e erros quadráticos médios das estimativas dos parâmetros do termo de cura para o modelo semiparamétrico - Alto percentual de omissão (50\%)

\begin{tabular}{lcccccc}
\hline & \multicolumn{3}{c}{ Vício } & \multicolumn{3}{c}{ EQM } \\
\hline Variável & ACC & Logísticos & FC & ACC & Logísticos & FC \\
\hline Sexo & 0,0094 & 0,0653 & $-0,0370$ & 0,2554 & 0,3248 & 0,3508 \\
Idade & 0,0032 & 0,0079 & $-0,0010$ & $7,94 \times 10^{-4}$ & $7,78 \times 10^{-4}$ & $1,74 \times 10^{-3}$ \\
Chagas & 0,4017 & 0,4477 & 0,6440 & 0,6641 & 0,7624 & 1,3707 \\
Feve & $-0,0201$ & $-0,0296$ & $-0,0082$ & $2,07 \times 10^{-3}$ & $4,33 \times 10^{-3}$ & $1,58 \times 10^{-3}$ \\
Intercepto & $-1,7477$ & $-0,4215$ & $-1,8449$ & 6,8836 & 4,9590 & 9,6254 \\
\hline
\end{tabular}




\subsubsection{Comparação entre os cenários}

Com o propósito de avaliar o efeito do percentual de omissão sobre a estimação dos parâmetros, foi realizada uma comparação entre os cenários $(10 \%, 30 \%$ e $50 \%$ de omissão). Para tanto, foram construídos gráficos para ilustrar o desempenho dos três métodos em termos de vício absoluto e erro quadrático médio.

A Figura 4.1 traz os gráficos dos vícios observados para os parâmetros do modelo de sobrevivência. Os gráficos reforçam a superioridade do método de pesos logísticos, que produziu estimativas, em média, menos viciadas em todos os cenários para todas as covariáveis consideradas.

O método de pesos FC apresentou pior desempenho que a ACC nos parâmetros referentes às covariáveis sexo e idade, independentemente do percentual de omissão. Para a variável Chagas, a ponderação FC foi um pouco melhor que a ACC nos percentuais maiores de omissão (30\% e 50\%).

No cenário de baixa omissão, os vícios das estimativas observados nos métodos ACC e pesos logísticos estão muito próximos. Com o aumento do percentual de omissão, a ACC torna-se bem menos eficiente que o método de pesos logísticos.

Com exceção da variável fração de ejeção, observou-se nos três métodos que o vício aumentou de acordo com o aumento do percentual de omissão. Curiosamente, para a variável fração de ejeção, a ACC apresentou menor vício no cenário de $50 \%$ de omissão do que no cenário de $30 \%$ de omissão. Para esta variável, o vício permaneceu praticamente inalterado no método de pesos FC. Uma possível explicação para este fato é que, neste método, a precisão das estimativas do modelo para estimar os pesos vai aumentando à medida que a desproporcionalidade entre omissos e não omissos vai diminuindo. Com isso, o maior vício esperado com o aumento das omissões e das censuras é contrabalanceado pela melhoria das estimativas para os pesos. 
vícIO - Modelo de Cox - SEXO

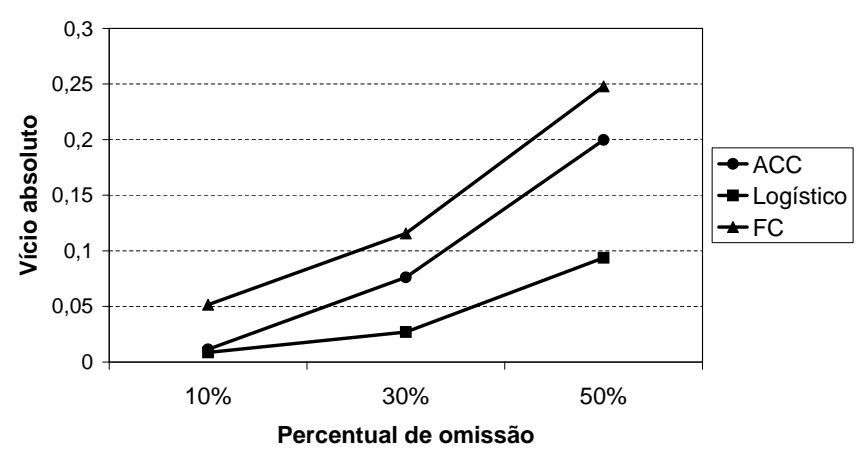

vícIO - Modelo de Cox - CHAGAS

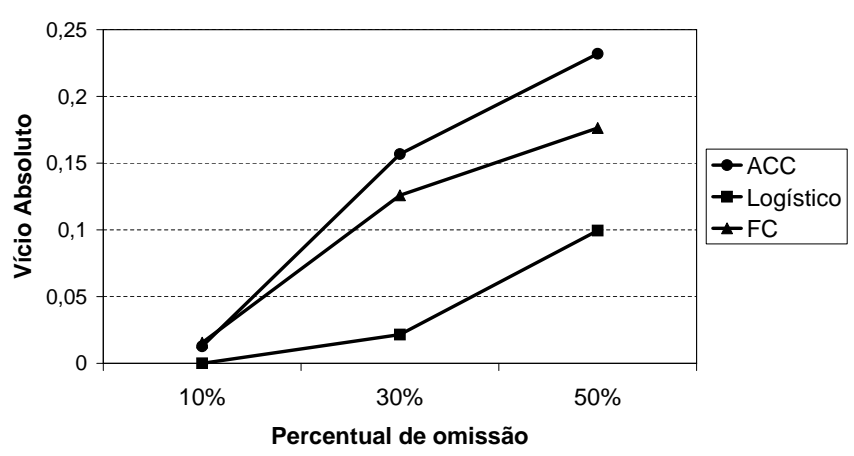

VícIO - Modelo de Cox - IDADE

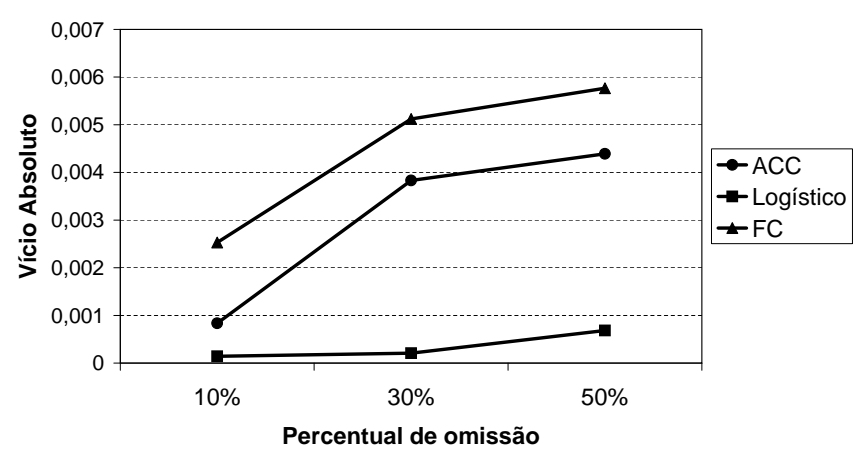

VícIo - Modelo de Cox - FEVE

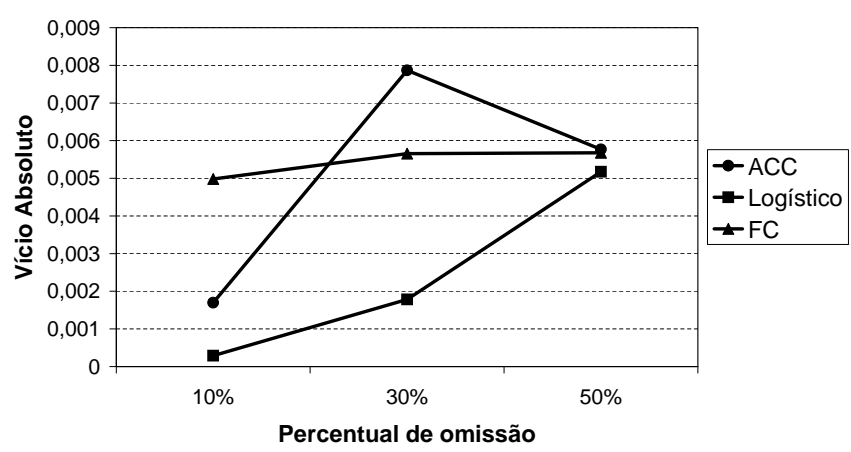

Figura 4.1. Vício absoluto das estimativas do modelo de Cox produzidas pelos três métodos segundo o percentual de omissão.

Em termos de erro quadrático médio (Figura 4.2), observa-se o esperado em todos os métodos, ou seja, o erro aumenta com o aumento do percentual de omissão.

As menores diferenças entre os métodos foram observadas na variável fração de ejeção, sendo que praticamente não houve diferença no cenário de alta omissão. Nas outras variáveis, o método de pesos logísticos supera os outros dois métodos à medida em que o percentual de omissões vai aumentando.

O método de pesos FC mostrou-se menos eficiente que a ACC para as variáveis sexo e idade. Esta desvantagem não foi observada para a variável Chagas. 
EQM - Modelo de Cox - SEXO

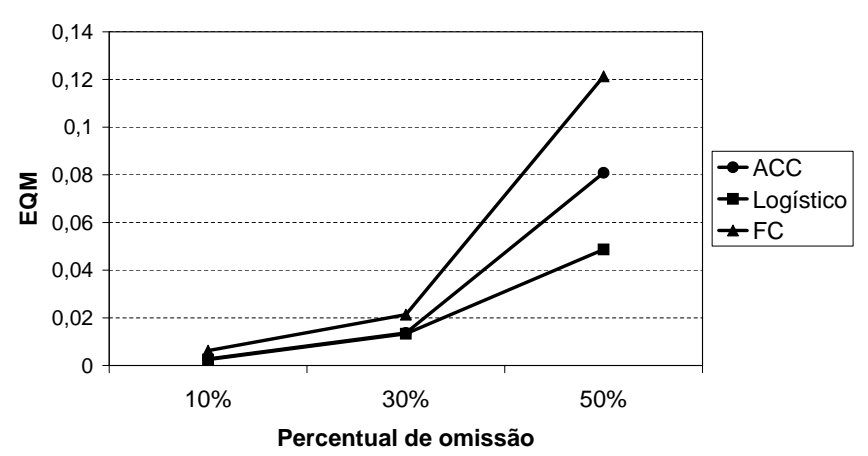

EQM - Modelo de Cox - CHAGAS

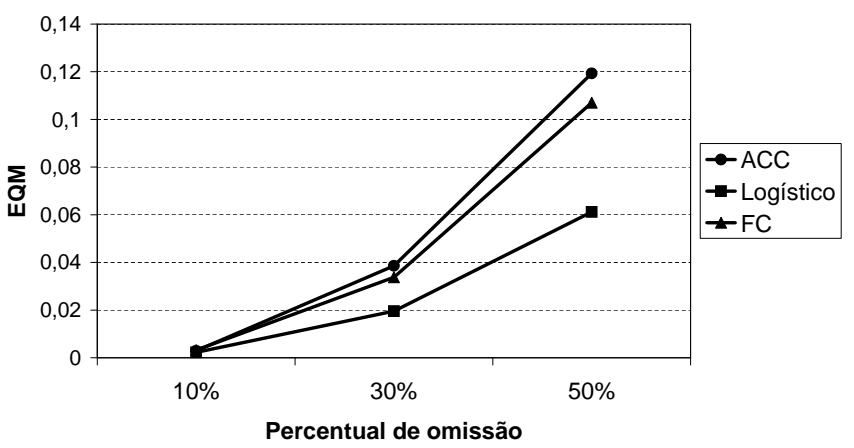

EQM - Modelo de Cox - IDADE

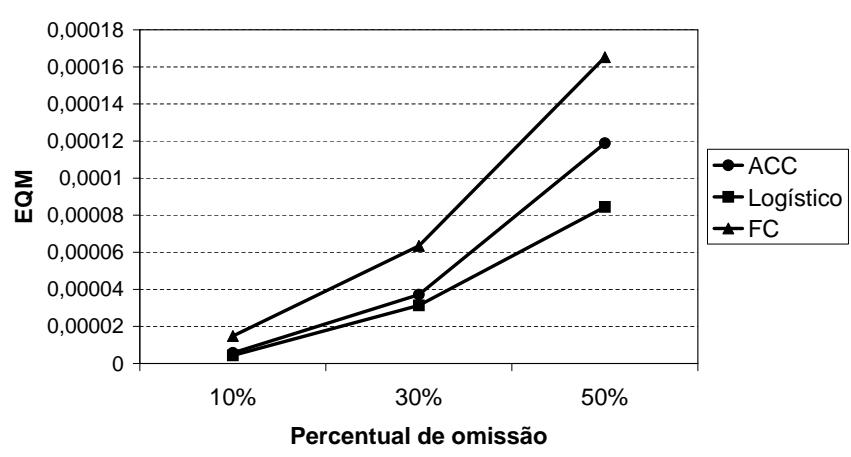

EQM - Modelo de Cox - FEVE

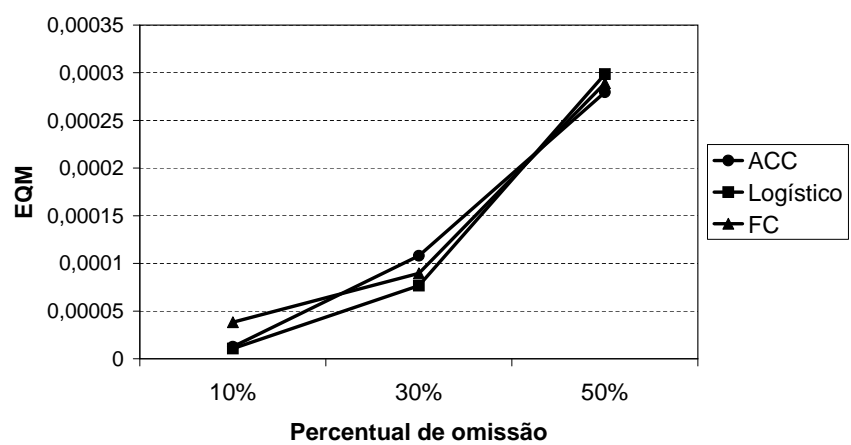

Figura 4.2. Erro quadrático médio das estimativas do modelo de Cox produzidas pelos três métodos segundo o percentual de omissão.

Com base no que foi observado nas simulações, verificou-se que a ponderação influencia muito mais as estimativas dos parâmetros relacionados à fração de cura do que as estimativas dos parâmetros relacionados aos tempos de sobrevivência. Comparativamente ao modelo de sobrevivência, no modelo de cura, as estimativas são consideravelmente mais viciadas.

Nos cenários de baixa e média omissão, praticamente não houve diferença entre o método de pesos logísticos e a ACC (Figura 4.3). No cenário de alta omissão, com exceção da variável Chagas, o método de pesos logísticos mostrou-se pior que a ACC em termos de vício. 
Quando se considerou apenas $10 \%$ de omissões, o vício observado no método de pesos FC foi bem superior aos outros dois métodos. Para as variáveis sexo, idade e Chagas, ao contrário do esperado, observa-se uma redução considerável do vício nos cenários de $30 \%$ e $50 \%$ de omissões. Conforme comentado anteriormente, esta redução pode ser devida à melhora das estimativas para os pesos FC.

No cenário de alta omissão, para a variável fração de ejeção, nota-se um grande aumento nos vícios produzidos pelos métodos ACC e pesos logísticos. O mesmo não acontece no método de pesos FC em que observa-se um aumento bem mais discreto.
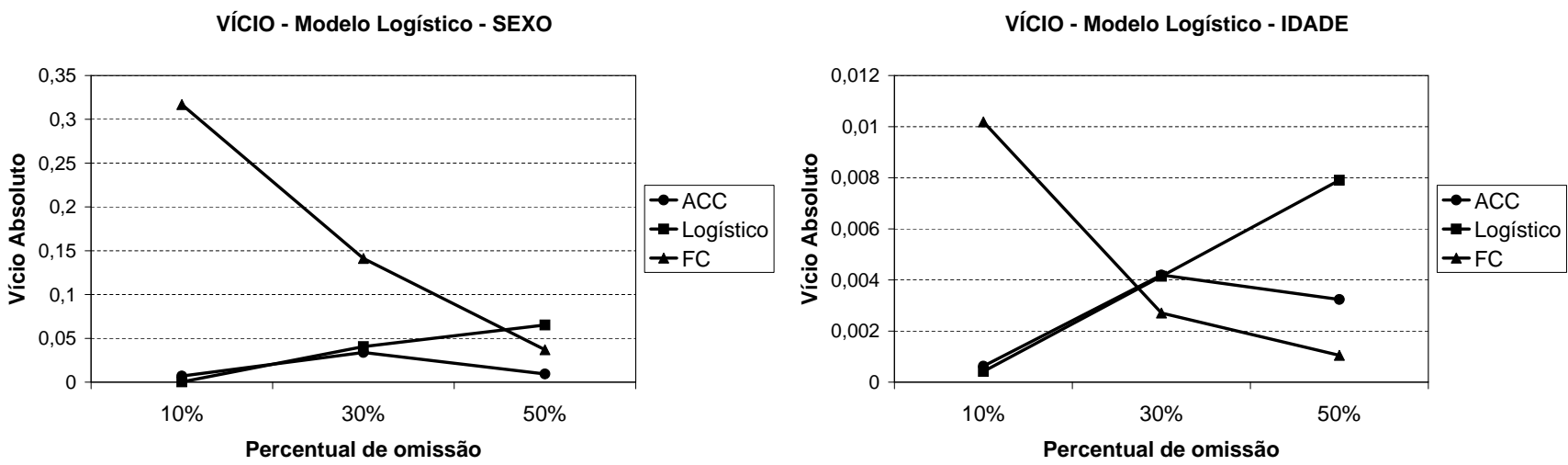

VícIO - Modelo Logístico - CHAGAS
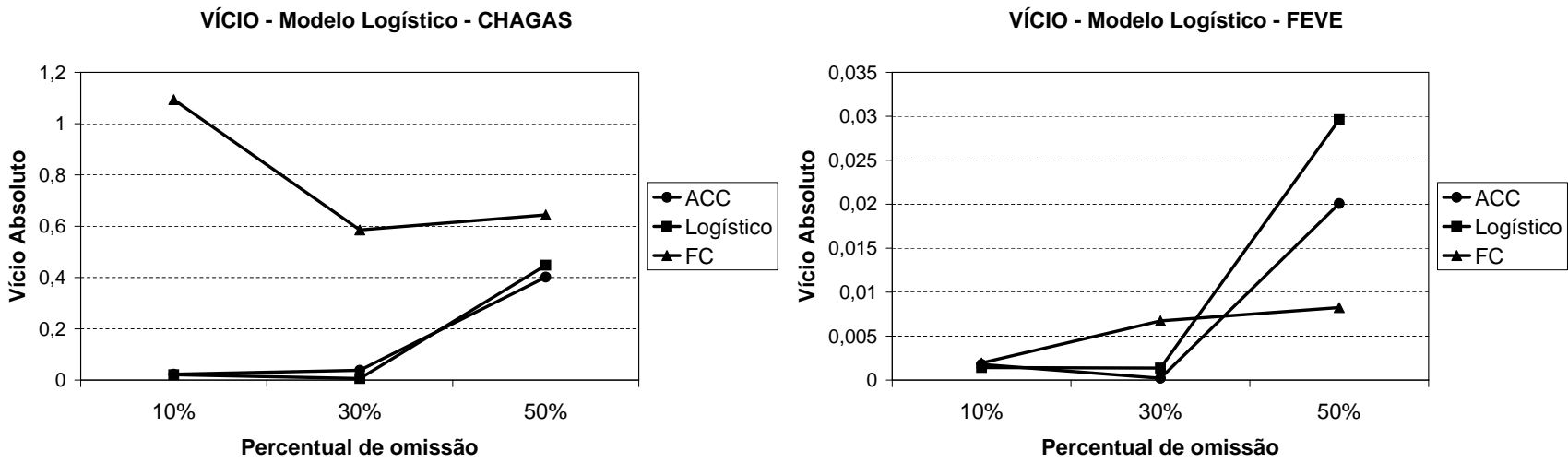

Figura 4.3. Vício absoluto das estimativas do modelo logístico produzidas pelos 3 métodos segundo percentual de omissão 
EQM - Modelo Logístico - SEXO

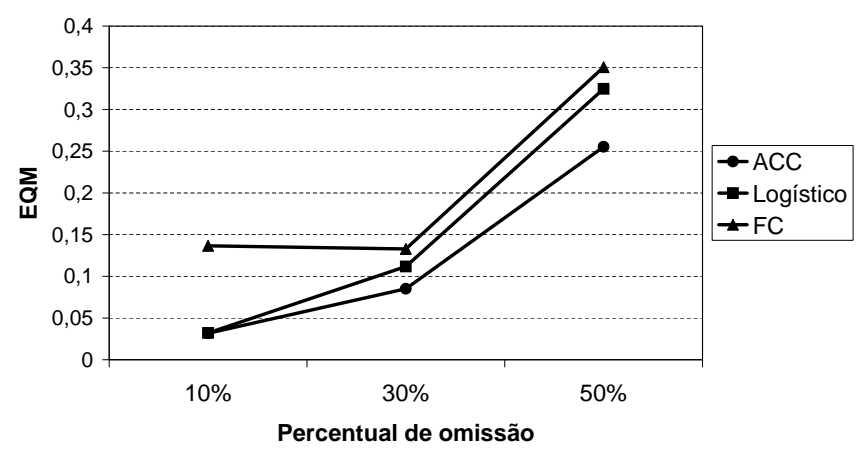

EQM - Modelo Logístico - CHAGAS

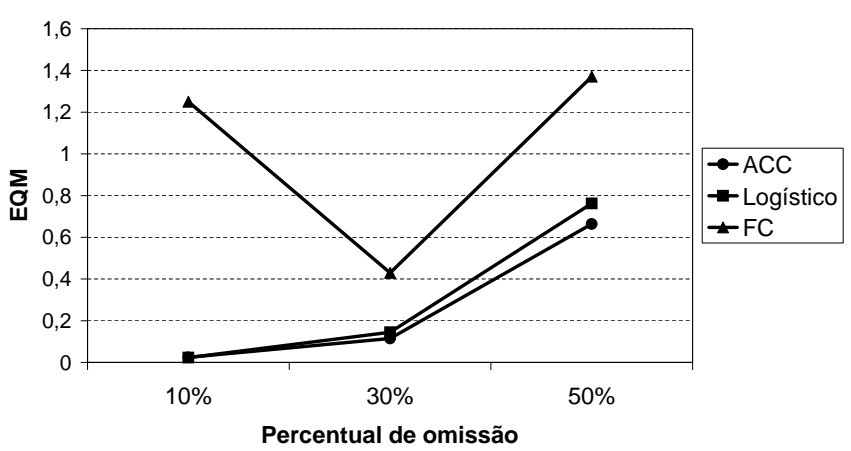

EQM - Modelo Logístico - IDADE

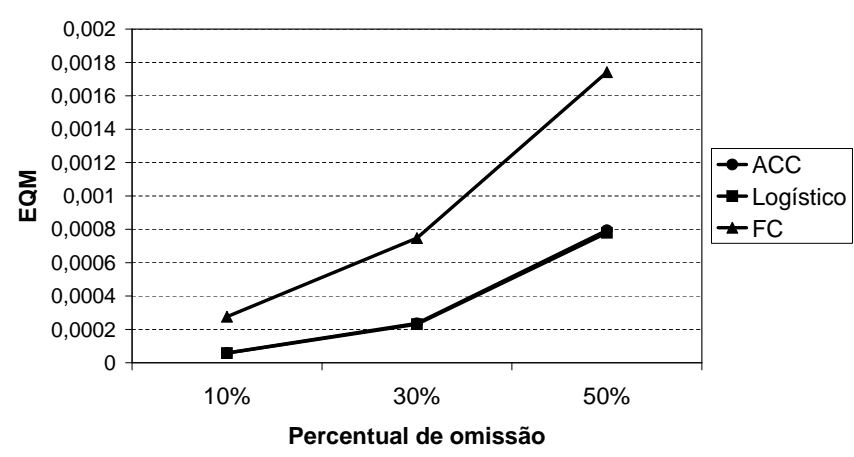

EQM - Modelo Logístico - FEVE

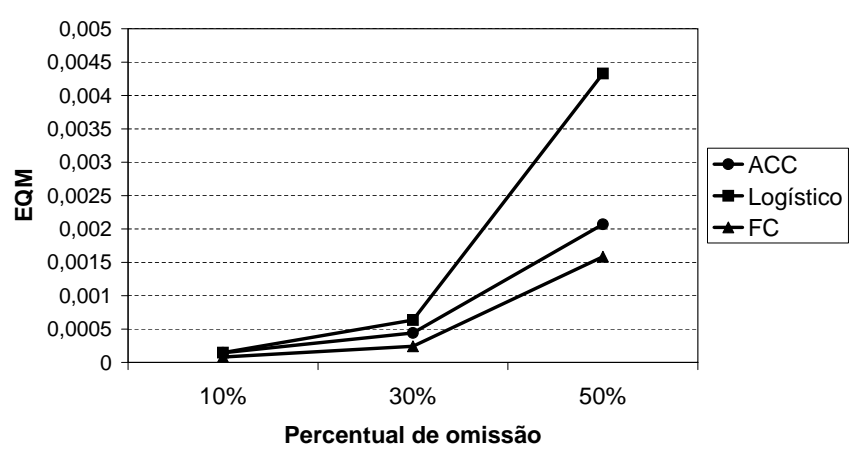

Figura 4.4. Erro quadrático médio das estimativas do modelo logístico produzidas pelos três métodos segundo o percentual de omissão.

Quanto ao erro quadrático médio, na maioria das situações o erro aumenta com o aumento do percentual de omissões. No cenário de baixa omissão, para a variável doença de Chagas, observou-se um EQM bastante elevado no método de pesos FC, que pode ser explicado novamente pela imprecisão das estimativas para os pesos. Este fato parece influenciar mais diretamente as variáveis qualitativas do que as quantitativas.

No termo de cura, para a variável fração de ejeção em que simulou-se dados omissos, foi observado melhor desempenho do método de pesos FC, principalmente no cenário de alta omissão. Neste cenário, o método de pesos logísticos apresentou EQM bem superior aos outros dois métodos. Nas demais variáveis, o método de pesos FC mostrouse inferior aos métodos ACC e pesos logísticos em todos os cenários. 


\section{Capítulo 5}

\section{Considerações finais e estudos futuros}

De um modo geral, para o conjunto de dados analisado neste trabalho, o modelo com pesos logísticos parece ser o mais adequado para modelar os tempos de sobrevivência, principalmente quando são considerados modelos com fração de cura semiparamétricos. No termo de cura, foram observados maiores vícios e imprecisão das estimativas em todos os modelos e freqüências de omissão consideradas.

Apesar de apresentar resultados coerentes nos modelos semiparamétricos, a ponderação por probabilidades de cura aparentemente não leva vantagem sobre a ponderação logística. Seu melhor desempenho foi no cenário de alto percentual de omissões nos parâmetros de cura relacionados às variáveis quantitativas.

Nos modelos paramétricos, as estimativas dos parâmetros relacionados às probabilidades de cura mostraram-se muito incoerentes do ponto de vista prático. Isto ocorreu em todas as freqüências de omissão, tanto nos modelos ponderados quanto na ACC. Mesmo fazendo parte da mesma função de verossimilhança, esta incoerência não foi observada nas estimativas dos parâmetros da parte de sobrevivência. Nestes modelos, utilizou-se a distribuição Weibull para modelar os tempos de sobrevivência, mas ou- 
tras distribuições podem ser utilizadas como, por exemplo, a distribuição Gama e a Lognormal.

A instabilidade das estimativas observada nos modelos com fração de cura Weibull pode estar relacionada a características e particularidades dos próprios dados considerados neste trabalho, ou mesmo do esquema adotado para simular as omissões. Um estudo mais detalhado com dados simulados a partir de distribuições teóricas, que controle não só o percentual de omissões como também o percentual de censuras, pode ser sugerido para explicar melhor o comportamento dos estimadores e identificar problemas no processo de estimação dos parâmetros.

Um fator importante a ser considerado é quanto às suposições relacionadas às probabilidades de cura e omissão. Neste trabalho, assumiu-se que estas probabilidades dependiam apenas da resposta e das covariáveis que eram completamente observadas. Na verdade, esta suposição é mais forte do que a definida como MAR, que supõe que a omissão (ou a cura) depende de tudo que é observado, inclusive dos dados disponíveis nas covariáveis com omissão. Na literatura existe uma grande variedade de possíveis configurações para mecanismos de omissão e alguns estudos de simulação para comparar as diferentes propostas, levando-se em conta o ganho de eficiência e as dificuldades computacionais. Apesar disso, pouco se sabe sobre a magnitude do viés no caso de violações das suposições sobre os diferentes tipos de omissão. Na literatura sobre modelos com fração de cura, não foram encontradas outras propostas de modelagem para as probabilidades de cura além do modelo logístico. Ainda não foram exploradas as conseqüências das violações sobre a especificação deste modelo, utilizado na grande maioria dos estudos. Estes aspectos, tanto para omissão quanto para a cura, não foram abordados neste trabalho, mas merecem atenção em estudos futuros.

Uma crítica freqüente em relação aos métodos de ponderação é que, mesmo que os pesos considerem a amostra total, apenas os indivíduos sem dados omissos são 
considerados nos modelos finais ponderados. Robins et al. (1994) sugerem outro tipo de equações de estimação ( WEE2) que acrescenta na função de estimação um termo que inclui os indivíduos com dados omissos. Esta proposta pode ser adaptada para modelos de Cox e modelos com fração de cura com omissão nas covariáveis.

O caminho mais promissor para estudos futuros é desenvolver a teoria assintótica para modelos com fração de cura ponderados. Neste trabalho foram delineados alguns passos que precisam ser mais explorados, mas outras alternativas podem ser consideradas. O estudo de Fang et al. (2005), por exemplo, utiliza outra metodologia para demonstrar a consistência e normalidade assintótica dos estimadores de máxima verossimilhança em modelos com fração de cura semiparamétricos não ponderados. Embora eles utilizem a ortogonalidade dos parâmetros, a teoria é desenvolvida para o modelo como um todo, sem separar o modelo para os tempos de sobrevivência do modelo para fração de cura. Recentemente, Zhao \& Zhou (2006) propuseram um novo modelo semiparamétrico com fração de cura, considerando uma estrutura de riscos proporcionais. Para o estudo assintótico, eles utilizam a teoria de processos de contagem e martingais. Em outro contexto, Lu \& Ying (2004) propõem uma classe mais geral de modelos de cura semiparamétricos baseada em equações de estimação generalizadas e usam a teoria de martingais para estudar as propriedades assintóticas dos estimadores propostos. Esta abordagem e a de Zhao \& Zhou (2006), embora utilizem modelagens diferentes da considerada neste trabalho, parecem muito interessantes principalmente por utilizarem a teoria de martingais, já consagrada para o modelo de Cox, mas ainda pouco explorada na análise de dados com fração de curados.

Em resumo, observou-se que quando existe um pior prognóstico para pacientes com omissão e uma alta proporção de censuras, faz sentido utilizar modelos com fração de cura ponderados. Na maioria das situações, o método de pesos logísticos mostrase mais adequado do que os pesos FC. Entretanto, da mesma forma que observado 
por Therneau \& Grambsch (2000) para o caso do modelo de Cox usual, em geral não existe um método mais adequado para lidar com dados omissos e a ponderação pode tanto melhorar quanto até piorar a estimação dos parâmetros. Estudos de simulação e análise de sensibilidade dos métodos propostos podem ser muito úteis para direcionar a escolha da melhor metodologia em cada situação. 


\section{Apêndice A}

\section{Derivadas de $2^{a}$ ordem}

Calculando as derivadas de segunda ordem do logaritmo da função de verossimilhança dada em (2.2), tem-se que a matriz de informação $\mathbf{F}_{n}(\boldsymbol{\theta})$ pode ser escrita como

$$
\mathbf{F}_{n}(\boldsymbol{\theta})=\sum_{i=1}^{n} \mathbf{Z}_{\mathbf{i}} \mathcal{F}_{i}(\boldsymbol{\theta}) \mathbf{Z}_{\mathbf{i}}{ }^{\prime}
$$

em que $\mathcal{F}_{i}(\boldsymbol{\theta})$ é uma matriz simétrica de dimensão $3 \times 3$, cujas componentes $f_{i}^{j k}$, $j, k=1,2,3$ são dadas por

$$
\begin{aligned}
f_{i}^{11}(\boldsymbol{\theta})= & -\Delta_{i} \delta^{2}\left(\lambda_{i} t_{i}\right)^{\delta}-\left(1-\Delta_{i}\right) \\
\times & \left\{\frac{\pi_{i}\left(\lambda_{i} t_{i}\right)^{\delta} \delta^{2} \mathrm{e}^{-\left(\lambda_{i} t_{i}\right)^{\delta}}\left(1-\left(\lambda_{i} t_{i}\right)^{\delta}\right)}{1-\pi_{i}\left(1-\mathrm{e}^{-\left(\lambda_{i} t_{i}\right)^{\delta}}\right)}-\left[\frac{\pi_{i}\left(\lambda_{i} t_{i}\right)^{\delta} \delta \mathrm{e}^{-\left(\lambda_{i} t_{i}\right)^{\delta}}}{1-\pi_{i}\left(1-\mathrm{e}^{-\left(\lambda_{i} t_{i}\right)^{\delta}}\right)}\right]^{2}\right\} \\
f_{i}^{22}(\boldsymbol{\theta})= & -\Delta_{i} \pi_{i}\left(1-\pi_{i}\right)-\left(1-\Delta_{i}\right) \\
& \times\left\{\frac{\left(\lambda_{i} t_{i}\right)^{\delta}\left(3 \pi_{i}^{2}-\pi_{i}-2 \pi_{i}^{3}\right)}{1-\pi_{i}\left(1-\mathrm{e}^{-\left(\lambda_{i} t_{i}\right)^{\delta}}\right)}-\left[\frac{\pi_{i}\left(1-\pi_{i}\right)\left(\lambda_{i} t_{i}\right)^{\delta}}{1-\pi_{i}\left(1-\mathrm{e}^{-\left(\lambda_{i} t_{i}\right)^{\delta}}\right)}\right]^{2}\right\}
\end{aligned}
$$




$$
\begin{aligned}
& f_{i}^{33}(\boldsymbol{\theta})=-\Delta_{i}\left(\frac{1}{\delta^{2}}+\left(\log \left(\lambda_{i} t_{i}\right)\right)^{2}\left(\lambda_{i} t_{i}\right)^{\delta}\right)-\left(1-\Delta_{i}\right) \\
& \times\left\{\frac{\pi_{i}\left(\lambda_{i} t_{i}\right)^{\delta} \mathrm{e}^{-\left(\lambda_{i} t_{i}\right)^{\delta}}\left(\log \left(\lambda_{i} t_{i}\right)\right)^{2}\left(1-\left(\lambda_{i} t_{i}\right)^{\delta}\right)}{1-\pi_{i}\left(1-\mathrm{e}^{-\left(\lambda_{i} t_{i}\right)^{\delta}}\right)}+\left[\frac{\pi_{i}\left(\lambda_{i} t_{i}\right)^{\delta} \mathrm{e}^{-\left(\lambda_{i} t_{i}\right)^{\delta}} \log \left(\lambda_{i} t_{i}\right)}{1-\pi_{i}\left(1-\mathrm{e}^{-\left(\lambda_{i} t_{i}\right)^{\delta}}\right)}\right]^{2}\right\} \\
& f_{i}^{12}(\boldsymbol{\theta})=\left(1-\Delta_{i}\right)\left\{\frac{\pi_{i} \delta\left(\lambda_{i} t_{i}\right)^{\delta} \mathrm{e}^{-\left(\lambda_{i} t_{i}\right)^{\delta}}\left(\pi_{i}-1\right)}{1-\pi_{i}\left(1-\mathrm{e}^{-\left(\lambda_{i} t_{i}\right)^{\delta}}\right)}-\frac{\pi_{i} \delta\left(\lambda_{i} t_{i}\right)^{\delta} \mathrm{e}^{-\left(\lambda_{i} t_{i}\right)^{\delta}}\left(1-\mathrm{e}^{-\left(\lambda_{i} t_{i}\right)^{\delta}}\right)\left(1-\pi_{i}\right)}{\left[1-\pi_{i}\left(1-\mathrm{e}^{-\left(\lambda_{i} t_{i}\right)^{\delta}}\right)\right]^{2}}\right\} \\
& f_{i}^{13}(\boldsymbol{\theta})=\Delta_{i}\left(1-\delta\left(\lambda_{i} t_{i}\right)^{\delta} \log \left(\lambda_{i} t_{i}\right)\right)+\left(1-\Delta_{i}\right) \\
& \times\left\{\frac{\pi_{i}\left(\lambda_{i} t_{i}\right)^{\delta} \mathrm{e}^{-\left(\lambda_{i} t_{i}\right)^{\delta}}\left(\delta \log \left(\lambda_{i} t_{i}\right)\left(\left(\lambda_{i} t_{i}\right)^{\delta}-1\right)-1\right)}{1-\pi_{i}\left(1-\mathrm{e}^{-\left(\lambda_{i} t_{i}\right)^{\delta}}\right)}-\delta \log \left(\lambda_{i} t_{i}\right)\left[\frac{\pi_{i}\left(\lambda_{i} t_{i}\right)^{\delta} \mathrm{e}^{-\left(\lambda_{i} t_{i}\right)^{\delta}}}{1-\pi_{i}\left(1-\mathrm{e}^{-\left(\lambda_{i} t_{i}\right)^{\delta}}\right)}\right]^{2}\right\} \\
& f_{i}^{23}(\boldsymbol{\theta})=\left(1-\Delta_{i}\right) \\
& \times\left\{\frac{\pi_{i}\left(\lambda_{i} t_{i}\right)^{\delta} \mathrm{e}^{-\left(\lambda_{i} t_{i}\right)^{\delta}} \log \left(\lambda_{i} t_{i}\right)\left(1-\pi_{i}\right)}{1-\pi_{i}\left(1-\mathrm{e}^{-\left(\lambda_{i} t_{i}\right)^{\delta}}\right)}\right. \\
& \left.-\pi_{i}\left(\lambda_{i} t_{i}\right)^{\delta} \mathrm{e}^{-\left(\lambda_{i} t_{i}\right)^{\delta}} \log \left(\lambda_{i} t_{i}\right)\left(\frac{\pi_{i}\left(1-\mathrm{e}^{-\left(\lambda_{i} t_{i}\right)^{\delta}}\right)\left(1+\pi_{i}\right)}{\left[1-\pi_{i}\left(1-\mathrm{e}^{-\left(\lambda_{i} t_{i}\right)^{\delta}}\right)\right]^{2}}\right)\right\}
\end{aligned}
$$




\section{Apêndice B}

\section{Algumas considerações sobre as propriedades assintóticas}

A seguir são discutidas algumas propriedades assintóticas dos estimadores de máxima verossimilhança em modelos com fração de cura, modelos lineares generalizados e modelos de Cox. A idéia é descrever alguns aspectos abordados nos métodos teóricos existentes, visando estendê-los para os estimadores ponderados propostos no capítulo anterior. O objetivo deste capítulo é fornecer diretrizes para a obtenção de possíveis resultados assintóticos a serem desenvolvidos em estudos futuros.

\section{B.1 Modelo Weibull}

Os principais artigos que propõem modelos paramétricos com fração de cura concentramse em métodos de estimação pontual dos parâmetros desconhecidos. Ghitany \& Maller (1992) foram os primeiros a analisar mais detalhadamente as propriedades assintóticas do estimador de máxima verossimilhança do modelo com fração de cura, supondo distribuição exponencial para os tempos de falha. Segundo Maller \& Zhou (1996), o esquema utilizado para o desenvolvimento teórico no modelo exponencial pode ser estendido para outras distribuições. Nesta seção a derivação de Ghitany et al. (1994) 
para os modelos com fração de cura sem ponderação considerando a distribuição Weibull é apresentada brevemente. Em seguida, discute-se a extensão do método para o caso em que as ponderações são incluídas.

Supõe-se que $n$ unidades experimentais são acompanhadas, sendo observado $X_{i}=$ $\min \left\{T_{i}, C_{i}\right\}$ e $\Delta_{i}=I_{\left(T_{i} \leq C_{i}\right)}$ em que $T_{i}$ são instantes de falha e $C_{i}$ são tempos de censura supostos independentes de $T_{i}$ e com distribuição que não depende de parâmetros associados aos tempos de falha. Supõe-se ainda que a cada observação estão associados vetores de covariáveis $\mathbf{Z}_{1 i}$ e $\mathbf{Z}_{2 i}$, em que $\mathbf{Z}_{1 i}$ está relacionado à distribuição dos tempos de falha e $\mathbf{Z}_{2 i}$ está relacionado à probabilidade de cura.

\section{B.1.1 Caso sem ponderação}

Supõe-se válida a família Weibull $\left(\lambda_{i}, \delta\right)$, com $\lambda_{i}>0$ e $\delta>0, i=1, \ldots, n$, para os tempos de falha das unidades experimentais. A verossimilhança dentro do contexto de modelos com fração de cura é

$$
L_{n}(\boldsymbol{\theta})=\prod_{i=1}^{n}\left(\pi_{i} \lambda_{i} \delta\left(\lambda_{i} t_{i}\right)^{\delta-1} \mathrm{e}^{-\left(\lambda_{i} t_{i}\right)^{\delta}}\right)^{\Delta_{i}}\left(1-\pi_{i}+\pi_{i} \mathrm{e}^{-\left(\lambda_{i} t_{i}\right)^{\delta}}\right)^{1-\Delta_{i}}
$$

em que $\pi_{i}$ é a probabilidade de não cura, parametrizada como

$$
\pi_{i}(\boldsymbol{\alpha})=\frac{\mathrm{e}^{\left(\boldsymbol{Z}_{2 i}^{\prime} \boldsymbol{\alpha}\right)}}{1+\mathrm{e}^{\left(\boldsymbol{Z}_{2 i}^{\prime} \boldsymbol{\alpha}\right)}}, \quad \boldsymbol{\alpha} \in \mathbb{R}^{r_{2}+1}
$$

e $\lambda_{i}$ o parâmetro de forma da Weibull, é parametrizado como

$$
\lambda_{i}(\boldsymbol{\beta})=\mathrm{e}^{\mathbf{Z}_{1 i}^{\prime} \boldsymbol{\beta}}, \quad \boldsymbol{\beta} \in \mathbb{R}^{r_{1}+1},
$$

sendo $\boldsymbol{\beta}$ e $\boldsymbol{\alpha}$ vetores de parâmetros desconhecidos associados a $\mathbf{Z}_{1 i}$ e $\mathbf{Z}_{2 i}$, respectivamente, $i=1, \ldots, n$. 
O estudo das propriedades do estimador de máxima verossimilhança dos modelos Weibull com fração de cura pode ser visto como uma extensão da teoria desenvolvida para modelos lineares generalizados (MLG, Fahrmeir \& Kaufmann (1985)).

No contexto de MLG, a formulação descrita para $\lambda_{i}$ corresponde a uma função de ligação $\log$-linear $\left(\log \lambda_{i}=\mathbf{Z}_{1 i}^{\prime} \boldsymbol{\beta}\right)$ e para o parâmetro $\pi_{i}$, a função de ligação é a logito $\left(\log \left(\pi_{i} /\left(1-\pi_{i}\right)\right)=\mathbf{Z}_{2 i}^{\prime} \boldsymbol{\alpha}\right)$.

Por simplicidade, considere que os parâmetros de interesse são colocados no vetor $\boldsymbol{\theta}=(\boldsymbol{\beta}, \boldsymbol{\alpha}, \delta)$ com dimensão $r \times 1, r=r_{1}+r_{2}+3$. Conforme foi visto no Capítulo 2 , a função escore e a matriz de informação referentes à função de verossimilhança dada em (B.1) podem ser escritos como

$$
\mathbf{U}_{n}(\boldsymbol{\theta})=\sum_{i=1}^{n} \mathbf{Z}_{i} \mathbf{s}_{i}(\boldsymbol{\theta}) \quad \text { e } \quad \mathbf{F}_{n}(\boldsymbol{\theta})=\sum_{i=1}^{n} \mathbf{Z}_{\mathbf{i}} \mathcal{F}_{i}(\boldsymbol{\theta}) \mathbf{Z}_{i}^{\prime}
$$

em que $\mathbf{Z}_{i}$ denota a matriz não estocástica dada por $\left(\begin{array}{ccc}\mathbf{Z}_{1 i} & 0 & 0 \\ 0 & \mathbf{Z}_{2 i} & 0 \\ 0 & 0 & 1\end{array}\right)_{r \times 3}$

$\mathbf{s}_{i}(\boldsymbol{\theta})$ é um vetor de dimensão $3 \times 1$ descrito no Capítulo 2 e $\mathcal{F}_{i}(\boldsymbol{\theta})$ é uma matriz aleatória simétrica de dimensão $3 \times 3$ cujos elementos $f_{i}^{r s}(\boldsymbol{\theta}), r, s=1,2$ e 3 são detalhados no Apêndice A. As componentes de $\mathbf{s}_{i}(\boldsymbol{\theta})$ e $\mathcal{F}_{i}(\boldsymbol{\theta})$ correspondem a funções dos tempos observados e dos parâmetros em $\boldsymbol{\theta}$.

Na teoria clássica de verossimilhança, se $\boldsymbol{\theta}_{0}$ é o verdadeiro valor do parâmetro e $\hat{\boldsymbol{\theta}}_{n}$ é seu estimador de máxima verossimilhança, a prova da convergência da distribuição assintótica de $\sqrt{n}\left(\hat{\boldsymbol{\theta}}_{n}-\boldsymbol{\theta}_{0}\right)$ baseia-se em duas principais propriedades: que $\mathbf{U}_{n}\left(\boldsymbol{\theta}_{0}\right)$ é uma soma de vetores aleatórios independentes com média zero e que a matriz $\mathbf{F}_{n}\left(\boldsymbol{\theta}_{0}\right)$ é positiva definida com esperança finita e que converge, em probabilidade, para a inversa da matriz de Informação de Fisher. 
A mesma metodologia pode ser utilizada para os modelos de sobrevivência com fração de cura. Ghitany et al. (1994) utilizam a independência entre os tempos de falha e censura para mostrar que $\mathrm{E}\left\{\mathbf{U}_{n}\left(\boldsymbol{\theta}_{0}\right)\right\}=0$ e que a matrix informação de Fisher, $\mathbf{D}_{n}$, dada por $\mathbf{D}_{n}=\mathrm{E}\left\{\mathbf{F}_{n}\left(\boldsymbol{\theta}_{0}\right)\right\}$, satisfaz $\mathbf{D}_{n}=\mathrm{E}\left\{\mathbf{U}_{n}\left(\boldsymbol{\theta}_{0}\right) \mathbf{U}_{n}^{\prime}\left(\boldsymbol{\theta}_{0}\right)\right\}$.

Usando (B.2) pode-se escrever

$$
\begin{aligned}
\mathbf{D}_{n} & =-E\left(\frac{\partial^{2} \log L_{n}\left(\boldsymbol{\theta}_{0}\right)}{\partial \boldsymbol{\theta}^{2}}\right) \\
& =E\left(\mathbf{F}_{n}\left(\boldsymbol{\theta}_{0}\right)\right) \\
& =\sum_{i=1}^{n} \mathbf{Z}_{\mathbf{i}} E\left(\mathcal{F}_{i}\left(\boldsymbol{\theta}_{0}\right)\right) \mathbf{Z}_{\mathbf{i}}{ }^{\prime}=\sum_{i=1}^{n} \mathbf{Z}_{\mathbf{i}} \mathcal{D}_{i} \mathbf{Z}_{\mathbf{i}}{ }^{\prime}
\end{aligned}
$$

em que $\boldsymbol{\theta}_{0}$ é o verdadeiro valor do parâmetro $\boldsymbol{\theta}$ e $\mathcal{D}_{i}=\mathrm{E}\left\{\mathcal{F}_{i}\left(\boldsymbol{\theta}_{0}\right)\right\}, i=1, \ldots, n$, são matrizes simétricas não aleatórias.

A distribuição assintótica de $\mathbf{D}_{n}^{-1 / 2} \mathbf{U}_{n}\left(\boldsymbol{\theta}_{0}\right)$ segue do teorema limite central de LindebergFeller (Sen \& Singer (1993)). Sob a suposição de que os tempos de censura são independentes e identicamente distribuídos e sob a condição que $\mathbf{D}_{n}^{-1}$ exista para $n$ suficientemente grande, Ghitany et al. (1994) mostram que o estimador de máxima verossimilhança $\hat{\boldsymbol{\theta}}$ é consistente para $\boldsymbol{\theta}_{0}$ e que

$$
\mathbf{D}_{n}^{-1 / 2}\left(\hat{\boldsymbol{\theta}_{n}}-\boldsymbol{\theta}_{0}\right) \stackrel{\mathcal{D}}{\longrightarrow} N\left(\mathbf{0}, \mathbf{I}_{r}\right)
$$

em que $\mathbf{I}_{r}$ é a matriz identidade de ordem $r$.

É importante observar que este resultado é restrito ao caso em que os parâmetros são interiores ao espaço paramétrico, mais especificamente em que as probabilidades de cura são positivas. Neste trabalho não serão abordadas situações nas quais os parâmetros estão na borda do espaço paramétrico, o que traz complicações técnicas nas expansões utilizadas nas demonstrações. 


\section{B.1.2 Família Weibull com ponderação}

Conforme discutido anteriormente, no modelo Weibull com fração de cura, a contribuição dos indivíduos na função escore é multiplicada por pesos individuais, que podem estar relacionados às probabilidades de omissão ou probabilidades de cura. Neste modelo, sob a suposição de que os pesos não dependem dos parâmetros $\boldsymbol{\theta}$ e que há omissão nas covariáveis, a função escore ponderada e a correspondente matriz de informação são expressas por:

$$
\mathbf{U}_{n, w}(\boldsymbol{\theta})=\sum_{i=1}^{n} R_{i} w_{i} \mathbf{Z}_{i} \mathbf{s}_{i}(\boldsymbol{\theta})
$$

$\mathrm{e}$

$$
\mathbf{F}_{n, w}(\boldsymbol{\theta})=-\frac{\partial \mathbf{U}_{n, w}(\boldsymbol{\theta})}{\partial \boldsymbol{\theta}}=\sum_{i=1}^{n} R_{i} w_{i} \mathbf{Z}_{i} \mathcal{F}_{i}(\boldsymbol{\theta}) \mathbf{Z}_{i}^{\prime}
$$

nas quais $w_{i}$ é o peso atribuído ao indivíduo $i, R_{i}$ é o indicador de dado sem omissão, $\mathbf{Z}_{\mathbf{i}}$ e $\mathbf{s}_{i}(\boldsymbol{\theta})$ são definidos no Capítulo 2 e $\mathcal{F}_{i}(\boldsymbol{\theta})$ é a matriz com elementos detalhados no Apêndice A.

Considere, inicialmente, o caso em que os pesos $w_{i}$ correspondem ao recíproco das probabilidades de dados sem omissão e suponha que a omissão depende apenas da resposta e das covariáveis com dados completos, ou seja, $w_{i}=1 / \xi_{i}$, tal que

$$
\begin{aligned}
\xi_{i} & =P\left(R_{i}=1 \mid X_{i}, \Delta_{i}, \mathbf{Z}_{i, \text { comp }}\right) \\
& =P\left(R_{i}=1 \mid X_{i}, \Delta_{i}, \mathbf{Z}_{i}\right) \\
& =\mathrm{E}\left(R_{i} \mid X_{i}, \Delta_{i}, \mathbf{Z}_{i}\right), \quad i=1, \ldots, n .
\end{aligned}
$$

Com esta suposição, pode-se verificar que a função escore ponderada é não viciada, pois para $\boldsymbol{\theta}=\boldsymbol{\theta}_{0}$ e usando (B.6), 


$$
\begin{aligned}
\mathrm{E}\left\{\mathbf{U}_{n, w}\left(\boldsymbol{\theta}_{0}\right)\right\} & =\mathrm{E}\left(\sum_{i=1}^{n} \frac{R_{i}}{\xi_{i}} \mathbf{Z}_{\mathbf{i}} \mathbf{s}_{i}\left(\boldsymbol{\theta}_{0}\right)\right) \\
& =\mathrm{E}\left(\mathrm{E}\left\{\sum_{i=1}^{n} \frac{R_{i}}{\xi_{i}} \mathbf{Z}_{i} \mathbf{s}_{i}\left(\boldsymbol{\theta}_{0}\right) \mid X_{i}, \Delta_{i}, \mathbf{Z}_{i}\right\}\right) \\
& =\mathrm{E}\left(\sum_{i=1}^{n} \frac{1}{\xi_{i}} \mathrm{E}\left\{R_{i} \mid X_{i}, \Delta_{i}, \mathbf{Z}_{i}\right\} \mathbf{Z}_{i} s_{i}\left(\boldsymbol{\theta}_{0}\right)\right) \\
& =\mathrm{E}\left(\sum_{i=1}^{n} \mathbf{Z}_{i} s_{i}\left(\boldsymbol{\theta}_{0}\right)\right) \\
& =\mathrm{E}\left(\mathbf{U}_{n}\left(\boldsymbol{\theta}_{0}\right)\right)=\mathbf{0},
\end{aligned}
$$

lembrando que $s_{i}(\boldsymbol{\theta})$ é função de $X_{i}, \Delta_{i}$ e de $\boldsymbol{\theta}, i=1, \ldots, n$. Este resultado pode ser utilizado para demonstrar a consistência do estimador de máxima verossimilhança ponderado $\boldsymbol{\theta}_{w}$ obtido pela equação $\mathbf{U}_{n, w}(\boldsymbol{\theta})=\mathbf{0}$.

A demonstração de Ghitany et al. (1994) desenvolvida para modelos com fração de cura sem ponderação está estreitamente relacionada aos métodos utilizados na teoria de modelos lineares generalizados. Utilizam-se expansões em Taylor, a convergência em probabilidade de $\mathbf{D}_{n}^{-1 / 2} \mathbf{F}_{n}(\boldsymbol{\theta}) \mathbf{D}_{n}^{-1 / 2}$ para uma matriz identidade e a convergência em distribuição da soma das variáveis aleatórias independentes $\mathbf{U}_{i}\left(\boldsymbol{\theta}_{0}\right)$, componentes da função escore $\mathbf{U}_{n}\left(\boldsymbol{\theta}_{0}\right)$. Uma possível abordagem é seguir os mesmos passos de Ghitany et al. (1994) estendendo a metodologia para modelos ponderados pelo inverso das probabilidades de não omissão, a exemplo do que foi feito por Robins et al. (1994) para modelos de regressão.

Para o caso em que os pesos correspondem ao recíproco das probabilidades de cura, a princípio pretende-se assumir que as probabilidades de cura $\xi_{i}$ podem ser aproximadas pelas probabilidades de não omissão, isto é, $\xi_{i}=P\left(R_{i} \mid X_{i}, \Delta_{i}, \mathbf{Z}_{i}\right)+1 / c_{n} \operatorname{com} c_{n} \rightarrow 0$ quando $n \rightarrow \infty$. Quando este não for o caso, tem-se uma função escore viciada, e a metodologia usual precisa ser adaptada para acomodar esta situação. 


\section{B.2 Modelo semiparamétrico}

A literatura que trata do estudo assintótico de estimadores em modelos com fração de cura semiparamétricos ainda é muito recente. Assim como nos modelos paramétricos, os artigos mais conhecidos concentram-se na estimação pontual, métodos computacionais e resultados numéricos.

No processo de estimação descrito anteriormente, os estimadores de máxima de verossimilhança no modelo semiparamétrico são obtidos a partir das componentes $L_{1} \mathrm{e}$ $L_{2}$ da função de verossimilhança, supondo que os indicadores de cura $\mathbf{b}=\left(b_{1}, \ldots, b_{n}\right)$ são observáveis. Neste modelo, dado b,

$$
L_{n}(\boldsymbol{\theta})=L_{1}(\boldsymbol{\alpha}) . L_{2}\left(\boldsymbol{\beta} ; \mathbf{S}_{N C}^{0}\right) \Rightarrow \log L_{n}(\boldsymbol{\theta})=\log L_{1}(\boldsymbol{\alpha})+\log L_{2}\left(\boldsymbol{\beta} ; \mathbf{S}_{N C}^{0}\right)
$$

em que $\boldsymbol{\alpha}$ é o parâmetro relacionado à probabilidade de cura, $\boldsymbol{\beta}$ e $\mathbf{S}_{N C}^{0}$ são os parâmetros relacionados à sobrevivência, $L_{1}$ é a função de verossimilhança do modelo logístico e $L_{2}$ é a função de verossimilhança para dados de sobrevivência sob o modelo de Cox.

Como $L_{1}$ só depende de $\boldsymbol{\alpha}$ e $L_{2}$ só depende de $\left(\boldsymbol{\beta} ; \mathbf{S}_{N C}^{0}\right)$, a matriz de informação é da forma diagonal em blocos, mais especificamente

$$
\mathbf{F}_{n}(\boldsymbol{\theta})=\left(\begin{array}{cc}
\mathbf{F}_{1}(\boldsymbol{\alpha}) & \mathbf{0} \\
\mathbf{0} & \mathbf{F}_{2}\left(\boldsymbol{\beta} ; \mathbf{S}_{N C}^{0}\right)
\end{array}\right)
$$

em que $\mathbf{F}_{1}(\boldsymbol{\alpha})=-\frac{\partial^{2} \log L_{1}}{\partial \boldsymbol{\alpha} \partial \boldsymbol{\alpha}^{\prime}} \quad$ e $\quad \mathbf{F}_{2}\left(\boldsymbol{\beta} ; \mathbf{S}_{N C}^{0}\right)=-\left(\begin{array}{cc}\frac{\partial^{2} \log L_{2}}{\partial \boldsymbol{\beta} \partial \boldsymbol{\beta}^{\prime}} & \frac{\partial^{2} \log L_{2}}{\partial \boldsymbol{\beta} \partial \mathbf{S}_{N C}^{0}} \\ \frac{\partial^{2} \log L_{2}}{\partial \mathbf{S}_{N C}^{0} \partial \boldsymbol{\beta}^{\prime}} & \frac{\partial^{2} \log L_{2}}{\partial \mathbf{S}_{N C}^{0} \partial \mathbf{S}_{N C}^{0 \prime}}\end{array}\right)$

o que implica que os parâmetros $\boldsymbol{\alpha}$ e $\left(\boldsymbol{\beta} ; \mathbf{S}_{N C}^{0}\right)$ são ortogonais. 
Devido à ortogonalidade, optou-se por analisar separadamente as duas verossimilhanças (modelo logístico e de Cox) e discutir as propriedades dos estimadores de máxima verossimilhança de cada um deles, levando-se em conta a ponderação.

\section{B.2.1 Modelo logístico para a probabilidade de cura}

No método proposto neste trabalho, o modelo logístico é utilizado em duas situações: a primeira para estimar os pesos $w_{i}=1 / \xi_{i}$, em que $\xi_{i}$ pode ser a probabilidade de não omissão ou a probabilidade de cura, e a segunda para estimar as probabilidades de não cura $\pi_{i}$ presentes no modelo final ponderado com base nos casos completos.

Para facilitar a descrição dos modelos ponderados, os pesos $w_{i}$ correspondentes aos recíprocos das probabilidades de dado completo estimadas por um modelo de regressão logística serão chamados de pesos logísticos. No caso em que os pesos são o recíproco das probabilidades de cura, estimadas por um modelo logístico como parte de um modelo com fração de cura, serão chamados de pesos $F C$.

Na primeira etapa (estimação dos pesos $w_{i}$ ), os pesos logísticos são estimados por um modelo de regressão logística usual, considerando como resposta os indicadores de dados completos $R_{i}$. Para os pesos FC, a resposta é o indicador de não cura $b_{i}$ e a estimação é baseada em um modelo com fração de cura no qual o modelo logístico é ajustado a cada iteração do algoritmo EM. Na segunda etapa (modelo final), ajusta-se um modelo com fração de cura que envolve em seu processo iterativo um modelo de regressão logística ponderado para estimar as probabilidades de não cura $\pi_{i}$.

Nas duas propostas de ponderação (logísticos ou FC), utiliza-se um modelo logístico sem ponderação na primeira etapa da estimação dos pesos e um modelo logístico ponderado na etapa final para a estimação das probabilidades de cura. Os modelos logísticos usual e ponderado, condicionados à observação de $b_{i}$, são discutidos a seguir. 


\section{Modelo logístico usual}

Considere o caso em que o modelo logístico faz parte de um modelo com fração de cura e é utilizado para estimar a probabilidade de não cura $\pi_{i}$.

Sejam $b_{i}, i=1, \ldots, n$, variáveis aleatórias independentes com distribuição Bernoulli $\left(\pi_{i}\right)$ que representam os indicadores de não cura. A função densidade de $b_{i}$ pode ser escrita na forma da família exponencial como

$$
f\left(b_{i} ; \theta_{i}, \phi\right)=\exp \left[\phi\left\{b_{i} \theta_{i}-g\left(\theta_{i}\right)\right\}+c\left(b_{i}, \phi\right)\right], \quad i=1, \ldots, n
$$

em que $\theta_{i}=\log \left\{\pi_{i} /\left(1-\pi_{i}\right)\right\}, g\left(\theta_{i}\right)=\log \left(1+\mathrm{e}^{\theta_{i}}\right), \phi=1$ e $c\left(b_{i}, \phi\right)=0$.

O modelo logístico, visto como caso particular da classe de Modelos Lineares Generalizados (MLG, McCullagh \& Nelder (1989)) é definido por (B.8) e pela função de ligação canônica $\theta_{i}=\log \left\{\pi_{i} /\left(1-\pi_{i}\right)\right\}=\mathbf{Z}_{i}^{\prime} \boldsymbol{\alpha}$, ou equivalentemente, $\pi_{i}=\frac{\mathrm{e}^{\mathbf{z}_{i}^{\prime} \boldsymbol{\alpha}}}{1+\mathrm{e}^{\mathbf{z}_{i}^{\prime} \boldsymbol{\alpha}}}$.

É simples ver que

$$
\mathrm{E}\left(b_{i}\right)=\pi_{i}=\pi_{i}\left(\theta_{i}\right)=\frac{\partial g\left(\theta_{i}\right)}{\partial \theta_{i}}, \quad \operatorname{Var}\left(b_{i}\right)=\pi_{i}\left(1-\pi_{i}\right)=\frac{\partial \pi_{i}}{\partial \theta_{i}}=\frac{\partial^{2} g\left(\theta_{i}\right)}{\partial \theta_{i}^{2}}
$$

O logaritmo da função de verossimilhança (em termos do parâmetro $\boldsymbol{\alpha}$ ) correspondente à expressão (B.8) e à função de ligação logito é

$$
\log L(\boldsymbol{\alpha})=\sum_{i=1}^{n}\left[b_{i} \theta_{i}-g\left(\theta_{i}\right)\right]=\sum_{i=1}^{n}\left[b_{i} \mathbf{Z}_{i}^{\prime} \boldsymbol{\alpha}-g\left(\mathbf{Z}_{i}^{\prime} \boldsymbol{\alpha}\right)\right]
$$

que resulta na função escore

$$
\mathbf{U}_{n}(\boldsymbol{\alpha})=\frac{\partial L(\boldsymbol{\alpha})}{\partial \boldsymbol{\alpha}}=\sum_{i=1}^{n}\left\{b_{i} \mathbf{Z}_{i}-\frac{\partial g\left(\mathbf{Z}_{i}^{\prime} \boldsymbol{\alpha}\right)}{\partial \boldsymbol{\alpha}}\right\}=\sum_{i=1}^{n}\left\{b_{i}-\pi_{i}(\boldsymbol{\alpha})\right\} \mathbf{Z}_{i}
$$


Para obter o estimador de máxima verossimilhança $\hat{\boldsymbol{\alpha}}$, resolve-se iterativamente a equação $\mathbf{U}_{n}(\boldsymbol{\alpha})=\mathbf{0}$, por exemplo, pelo método scoring de Fisher. Neste caso, a matriz de informação de Fisher é

$$
\mathbf{D}_{n}(\boldsymbol{\alpha})=\mathrm{E}\left\{-\frac{\partial^{2}}{\partial \boldsymbol{\alpha} \partial \boldsymbol{\alpha} \prime} \log L(\boldsymbol{\alpha})\right\}=\sum_{i=1}^{n} v_{i}(\boldsymbol{\alpha}) \mathbf{Z}_{i} \mathbf{Z}_{i}^{\prime}
$$

em que $v_{i}(\boldsymbol{\alpha})=\pi_{i}(\boldsymbol{\alpha})\left[1-\pi_{i}(\boldsymbol{\alpha})\right]$ e o estimador de $\boldsymbol{\alpha}$ é obtido pelo processo iterativo

$$
\boldsymbol{\alpha}^{(k+1)}=\boldsymbol{\alpha}^{(k)}+\mathbf{D}_{n}^{-1}\left(\boldsymbol{\alpha}^{(k)}\right) \mathbf{U}_{n}\left(\boldsymbol{\alpha}^{(k)}\right) .
$$

Sob condições gerais de regularidade (ver, por exemplo, Sen \& Singer (1993), Cap.7), $\hat{\boldsymbol{\alpha}}$ é um estimador consistente do verdadeiro valor $\boldsymbol{\alpha}_{0} \mathrm{e}$

$$
\sqrt{n}\left(\hat{\boldsymbol{\alpha}}-\boldsymbol{\alpha}_{0}\right) \stackrel{\mathcal{D}}{\longrightarrow} N\left(\mathbf{0}, \mathbf{D}^{-1}\left(\boldsymbol{\alpha}_{0}\right)\right)
$$

em que $\mathbf{D}(\boldsymbol{\alpha})=\lim _{n \rightarrow \infty} \frac{\mathbf{D}_{n}(\boldsymbol{\alpha})}{n}$, que pode ser consistentemente estimada por $\mathbf{D}(\hat{\boldsymbol{\alpha}})$.

\section{Modelo logístico ponderado}

No caso de omissão nas covariáveis, a função escore referente ao modelo logístico ponderado é

$$
\mathbf{U}_{n, w}(\boldsymbol{\alpha})=\sum_{i=1}^{n} R_{i} w_{i} \mathbf{U}_{i}(\boldsymbol{\alpha})=\sum_{i=1}^{n} R_{i} w_{i}\left\{b_{i}-\pi_{i}(\boldsymbol{\alpha})\right\} \mathbf{Z}_{i}
$$

no qual $R_{i}$ é a variável binária indicadora de dados completos (não omissão) e $w_{i}$ são os pesos atribuídos aos indivíduos. 
Considere o caso de ponderação pelas probabilidades de não omissão. Suponha que

$$
\begin{aligned}
\xi_{i} & =P\left(R_{i}=1 \mid \mathbf{Z}_{i, \text { comp }}\right)=P\left(R_{i}=1 \mid \mathbf{Z}_{i}\right) \\
& =\mathrm{E}\left(R_{i} \mid \mathbf{Z}_{i}\right), \quad i=1, \ldots, n .
\end{aligned}
$$

Neste caso, o estimador de máxima verossimilhança ponderado $\hat{\boldsymbol{\alpha}}_{w}$ é obtido como solução da equação

$$
\mathbf{U}_{n, w}\left(\hat{\boldsymbol{\alpha}}_{w}\right)=\sum_{i=1}^{n} \frac{R_{i}}{\xi_{i}} \mathbf{U}_{i}\left(\hat{\boldsymbol{\alpha}}_{w}\right)=\sum_{i=1}^{n} \frac{R_{i}}{\xi_{i}}\left\{b_{i}-\pi_{i}\left(\hat{\boldsymbol{\alpha}}_{w}\right)\right\} \mathbf{Z}_{i}=0 .
$$

A consistência do estimador ponderado vem do fato que a função escore ponderada é não viciada para zero no verdadeiro valor do parâmetro. Sob a suposição (B.11), tem-se que $\mathrm{E}\left\{\frac{R_{i}}{\xi_{i}} \mid \mathbf{Z}_{\mathbf{i}}\right\}=1, \log \mathrm{O}$

$$
\mathrm{E}\left[\mathbf{U}_{n, w}\left(\boldsymbol{\alpha}_{0}\right)\right]=\mathrm{E}\left[\mathrm{E}\left\{\sum_{i=1}^{n} \frac{R_{i}}{\xi_{i}} U_{i}\left(\boldsymbol{\alpha}_{0}\right) \mid \mathbf{Z}_{i}\right\}\right]=\mathrm{E}\left[\sum_{i=1}^{n} U_{i}\left(\boldsymbol{\alpha}_{0}\right)\right]=\mathrm{E}\left[\mathbf{U}_{n}\left(\boldsymbol{\alpha}_{0}\right)\right]=0
$$

As equações de estimação definidas por (4.12) são um caso particular da classe de equações de estimação ponderadas (WEE - Weighted Estimating Equations) sugeridas originalmente por Robins et al. (1994). Segundo Ibrahim et al. (2005), se os pesos são conhecidos ou consistentemente estimados, o estimador ponderado é assintoticamente normal e sua variância pode ser consistentemente estimada por um estimador sanduíche robusto (Zhao et al. (1996)).

No caso de pesos estimados com base em um modelo paramétrico $\xi(\gamma)$, o estimador consistente da variância assintótica de $\hat{\boldsymbol{\alpha}}_{w}(\hat{\gamma})$ que resolve a equação $\mathbf{U}_{n, w}(\boldsymbol{\alpha}, \hat{\gamma})=\mathbf{0}$ não é simples e depende da distribuição de $\hat{\gamma}$. A sugestão de Lipsitz et al. (1998) 
é utilizar o método de bootstrap ou jackknife (Efron (1982)). Outra alternativa é combinar as duas equações de estimação (dos modelos logísticos para $\xi$ e $\pi$ ) e obter um estimador sanduíche em termos de quantidades obtidas a partir da função de estimação combinada (Zhao et al. (1996)).

\section{B.2.2 Modelo de Cox para a sobrevivência dos não curados}

Neste trabalho, o modelo de Cox é utilizado dentro da formulação de modelos com fração de cura semiparamétricos. Os resultados assintóticos do modelo de Cox descritos a seguir são expressos sob a teoria de processos de contagem (Andersen et al. (1993)).

\section{Modelo de Cox usual}

Considere que $n$ pacientes são observados ao longo do tempo até a ocorrência de algum evento. Sejam $T_{1}, T_{2}, T_{3}, \ldots, T_{n}$ os tempos de falha desses indivíduos e $C_{1}, C_{2}, C_{3}, \ldots, C_{n}$ seus tempos de censura. Definindo $X_{i}=\min \left\{T_{i}, C_{i}\right\}$ e $\Delta_{i}=I_{\left\{T_{i} \leq C_{i}\right\}}$ (indicador de falha), o que é de fato observado pode ser convenientemente representado por um processo de contagem multivariado $\mathbf{N}(t)=\left\{N_{1}(t), \ldots, N_{n}(t)\right\}$, em que $N_{i}(t)=I_{\left\{X_{i} \leq t, \Delta_{i}=1\right\}}$.

No modelo de Cox usual (Cox (1972)), dado um vetor de covariáveis $\mathbf{Z}_{i}$, o parâmetro de interesse $\boldsymbol{\beta}$ é estimado maximizando a função de verossimilhança parcial (Cox $(1975))$

$$
L_{n}(\boldsymbol{\beta})=\boldsymbol{\pi}_{t \geq 0} \prod_{i=1}^{n}\left(\frac{\exp \left(\boldsymbol{\beta}^{\prime} \mathbf{Z}_{i}\right)}{\sum_{j=1}^{n} Y_{j}(t) \exp \left(\boldsymbol{\beta}^{\prime} \mathbf{Z}_{j}\right)}\right)^{d N_{i}(t)}
$$

em que $Y_{i}(t)=I_{\left\{X_{i} \geq t\right\}}$ indica se o indivíduo $i$ está ou não em risco no instante imedia- 
tamente anterior a $t$ e $d N_{i}(t)=N_{i}(t)-N_{i}(t-d t)$ é o incremento do processo $N_{i}(t)$,que corresponde ao indicador de falha para o indivíduo $i$.

Tomando o logaritmo da função de verossimilhança

$$
\begin{aligned}
l(\boldsymbol{\beta}) & =\log L_{n}(\boldsymbol{\beta}) \\
& =\sum_{i=1}^{n} \int_{0}^{\infty}\left\{\boldsymbol{\beta}^{\prime} \mathbf{Z}_{i}-\log \sum_{j=1}^{n} Y_{j}(s) \exp \left(\boldsymbol{\beta}^{\prime} \mathbf{Z}_{j}\right)\right\} d N_{i}(s) \\
& =\sum_{i=1}^{n} \int_{0}^{\infty} \boldsymbol{\beta}^{\prime} \mathbf{Z}_{i} d N_{i}(s)-\int_{0}^{\infty} \log \left\{\sum_{j=1}^{n} Y_{j}(s) \exp \left(\boldsymbol{\beta}^{\prime} \mathbf{Z}_{j}\right)\right\} d \bar{N}(s)
\end{aligned}
$$

em que $\bar{N}(t)=\sum_{i=1}^{n} N_{i}(t)$, a função escore relativa à verossimilhança parcial pode ser representada por

$$
\begin{aligned}
\mathbf{U}_{n}(\boldsymbol{\beta}) & =\frac{\partial l(\boldsymbol{\beta})}{\partial \boldsymbol{\beta}}=\frac{\partial \log L_{n}(\boldsymbol{\beta})}{\partial \boldsymbol{\beta}} \\
& =\sum_{i=1}^{n} \int_{0}^{\infty}\left\{\mathbf{Z}_{i}-\frac{\sum_{j=1}^{n} Y_{j}(s) \mathbf{Z}_{j} \exp \left(\boldsymbol{\beta}^{\prime} \mathbf{Z}_{j}\right)}{\sum_{j=1}^{n} Y_{j}(s) \exp \left(\boldsymbol{\beta}^{\prime} \mathbf{Z}_{j}\right)}\right\} d N_{i}(s) .
\end{aligned}
$$

O estimador de máxima verossimilhança parcial é obtido resolvendo a equação escore $\mathbf{U}_{n}(\hat{\boldsymbol{\beta}})=\mathbf{0}$. Da mesma forma que na teoria de verossimilhança usual, o estimador da matriz de covariância de $\hat{\boldsymbol{\beta}}$ é obtido a partir da matriz de informação

$$
\begin{aligned}
\mathbf{I}(\boldsymbol{\beta}) & =-\frac{\partial^{2} \log L_{n}(\boldsymbol{\beta})}{\partial \boldsymbol{\beta}^{2}}=-\frac{\partial \mathbf{U}_{n}(\boldsymbol{\beta}, t)}{\partial \boldsymbol{\beta}} \\
& =\int_{0}^{\infty}\left(\frac{\sum_{j=1}^{n} Y_{j}(s) \mathbf{Z}_{j}^{\otimes 2} \exp \left(\boldsymbol{\beta}^{\prime} \mathbf{Z}_{j}\right)}{\sum_{j=1}^{n} Y_{j}(s) \exp \left(\boldsymbol{\beta}^{\prime} \mathbf{Z}_{j}\right)}-\left(\frac{\sum_{j=1}^{n} Y_{j}(s) \mathbf{Z}_{j} \exp \left(\boldsymbol{\beta}^{\prime} \mathbf{Z}_{j}\right)}{\sum_{j=1}^{n} Y_{j}(s) \exp \left(\boldsymbol{\beta}^{\prime} \mathbf{Z}_{j}\right)}\right)^{\otimes 2}\right) d \bar{N}(s)
\end{aligned}
$$

em que $\bar{N}(t)=\sum_{i=1}^{n} N_{i}(t)$ e para um vetor $\mathbf{a}, \mathbf{a}^{\otimes 2}=\mathbf{a a}^{\prime}$.

Para escrever a função escore e a matriz de covariâncias de forma conveniente, pode-se utilizar a seguinte notação: 


$$
S^{(r)}(\boldsymbol{\beta}, t)=n^{-1} \sum_{i=1}^{n} Y_{i}(t) e^{\boldsymbol{\beta}^{\prime} \mathbf{Z}_{i}} \mathbf{Z}_{i}^{\otimes r}
$$

Logo, $S^{(0)}(\boldsymbol{\beta}, t)=n^{-1} \sum_{i=1}^{n} Y_{i}(t) e^{\boldsymbol{\beta}^{\prime} \mathbf{Z}_{i}} \quad, S^{(1)}(\boldsymbol{\beta}, t)=n^{-1} \sum_{i=1}^{n} Y_{i}(t) e^{\boldsymbol{\beta}^{\prime} \mathbf{Z}_{i}} \mathbf{Z}_{i} \quad \mathrm{e}$ $S^{(2)}(\boldsymbol{\beta}, t)=n^{-1} \sum_{i=1}^{n} Y_{i}(t) e^{\boldsymbol{\beta}^{\prime} \mathbf{Z}_{i}} \mathbf{Z}_{i}^{\otimes 2}$.

Defina

$$
\overline{\mathbf{Z}}(\boldsymbol{\beta}, t)=\frac{S^{(1)}(\boldsymbol{\beta}, t)}{S^{(0)}(\boldsymbol{\beta}, t)} \quad \text { e } \quad \mathrm{V}(\boldsymbol{\beta}, t)=\frac{S^{(2)}(\boldsymbol{\beta}, t)}{S^{(0)}(\boldsymbol{\beta}, t)}-\overline{\mathbf{Z}}(\boldsymbol{\beta}, t)^{\otimes 2}
$$

Com esta notação, pode-se reescrever a função escore e a matriz de informação como

$$
\begin{gathered}
\mathbf{U}_{n}(\boldsymbol{\beta})=\sum_{i=1}^{n} \int_{0}^{\infty}\left\{\mathbf{Z}_{i}-\frac{S^{(1)}(\boldsymbol{\beta}, s)}{S^{(0)}(\boldsymbol{\beta}, s)}\right\} d N_{i}(s) \\
=\sum_{i=1}^{n} \int_{0}^{\infty}\left\{\mathbf{Z}_{i}-\overline{\mathbf{Z}}(\boldsymbol{\beta}, s)\right\} d N_{i}(s) \\
\mathbf{I}(\boldsymbol{\beta})=\sum_{i=1}^{n} \int_{0}^{\infty}\left(\frac{S^{(2)}(\boldsymbol{\beta}, s)}{S^{(0)}(\boldsymbol{\beta}, s)}-\left(\frac{S^{(1)}(\boldsymbol{\beta}, s)}{S^{(0)}(\boldsymbol{\beta}, s)}\right)^{\otimes 2}\right) d N_{i}(s) \\
=\sum_{i=1}^{n} \int_{0}^{\infty} \mathrm{V}(\boldsymbol{\beta}, s) d N_{i}(s)
\end{gathered}
$$

Notações deste tipo são muito comuns em estudos sobre o modelo de Cox e visam facilitar a interpretação e desenvolvimento da teoria assintótica.

Para fazer inferências sobre o estimador de máxima veossimilhança parcial, Andersen \& Gill (1982) utilizam a teoria de martingais (Andersen et al. (1993)). O primeiro 
passo é mostrar que a estatística escore da função de verossimilhança parcial é um martingal de média zero.

Defina o processo martingal $M_{i}(t)=N_{i}(t)-\int_{0}^{t} Y_{i}(s) h(s) d s, t \geq 0$, ou equivalentemente, $d M_{i}(t)=d N_{i}(t)-Y_{i}(t) h_{i}(t) d t$, em que $h(t)$ é a função de risco. Pela propriedade martingal (Andersen et al. (1993)) tem-se que para qualquer $t, M_{i}(t)$ tem média zero e seus incrementos são não correlacionados. Este resultado é fundamental para determinar a distribuição assintótica do processo escore.

Se a função de risco é especificada por um modelo de Cox, isto é, $h(t)=h_{0}(t) \mathrm{e}^{\beta^{\prime} Z_{i}}$, tem-se que $d N_{i}(t)=d M_{i}(t)+Y_{i}(t) h_{0}(t) \mathrm{e}^{\beta^{\prime} Z_{i}}$. Assim, pode-se reescrever o processo escore como

$$
\mathbf{U}_{n}(\boldsymbol{\beta}, t)=\sum_{i=1}^{n} \int_{0}^{t}\left\{\mathbf{Z}_{i}-\overline{\mathbf{Z}}(\boldsymbol{\beta}, s)\right\} d M_{i}(s)
$$

A partir daí, verifica-se que o processo escore corresponde a uma soma de martingais de média zero e, portanto, também é um martingal de média zero. Com este resultado, Andersen \& Gill (1982) aplicam o teorema do limite central para martingais (Rebolledo (1980)) para mostrar que $n^{1 / 2} \mathbf{U}_{n}\left(\boldsymbol{\beta}_{0}, t\right)$ converge em distribuição para um processo gaussiano. Em seguida, usando expansão de Taylor, eles mostram a normalidade assintótica de $n^{1 / 2}\left(\hat{\boldsymbol{\beta}}-\boldsymbol{\beta}_{0}\right)$, cuja matriz de covariância $\left\{\mathrm{E}\left(\mathbf{I}\left(\boldsymbol{\beta}_{0}\right)\right)\right\}^{-1}$, pode ser consistentemente estimada por $(\mathbf{I}(\hat{\boldsymbol{\beta}}))^{-1}$.

\section{Modelo de Cox ponderado}

A idéia de ponderar a função escore no modelo de Cox para incorporar informação sobre a omissão foi proposta inicialmente por Pugh et al. (1993), motivados pelas equações de estimação ponderadas de Robins et al. (1994), publicadas como relatório técnico em 1992. No modelo de Cox ponderado com omissão nas covariáveis, as somas 
na função escore $\mathbf{U}_{n}(\boldsymbol{\beta})$ são substituídas por somas ponderadas resultando na função escore parcial ponderada

$$
\mathbf{U}_{n, w}(\boldsymbol{\beta})=\sum_{i=1}^{n} \int_{0}^{\infty} R_{i} w_{i}\left\{\mathbf{Z}_{i}-\overline{\mathbf{Z}}_{w}(\boldsymbol{\beta}, s)\right\} d N_{i}(s)
$$

em que $R_{i}$ é o indicador de dados completos, $w_{i}$ é o peso atribuído ao indivíduo $i$ e $\overline{\mathbf{Z}}_{w}(\beta, t)=\frac{\sum_{j=1}^{n} R_{j} w_{j} Y_{j}(t) \exp \left\{\beta^{\prime} Z_{j}\right\} Z_{j}}{\Sigma_{j=1}^{n} R_{j} w_{j} Y_{j}(t) \exp \left\{\beta^{\prime} Z_{j}\right\}}$. Assim, o estimador de máxima verossimilhança ponderado é obtido resolvendo a equação $\mathbf{U}_{n, w}\left(\hat{\boldsymbol{\beta}}_{w}\right)=0$.

Usando notação análoga à da expressão (B.15) defina

$$
S_{w}^{(r)}(\boldsymbol{\beta}, t)=n^{-1} \sum_{i=1}^{n} R_{i} w_{i} Y_{i}(t) e^{\boldsymbol{\beta}^{\prime} \mathbf{Z}_{i}} \mathbf{Z}_{i}^{\otimes r}
$$

Logo,

$$
\begin{gathered}
S_{w}^{(0)}(\boldsymbol{\beta}, t)=n^{-1} \sum_{i=1}^{n} R_{i} w_{i} Y_{i}(t) e^{\boldsymbol{\beta}^{\prime} \mathbf{Z}_{i}}, \\
S_{w}^{(1)}(\boldsymbol{\beta}, t)=n^{-1} \sum_{i=1}^{n} R_{i} w_{i} Y_{i}(t) e^{\boldsymbol{\beta}^{\prime} \mathbf{Z}_{i}} \mathbf{Z}_{i} \quad \text { e } \\
S_{w}^{(2)}(\boldsymbol{\beta}, t)=n^{-1} \sum_{i=1}^{n} R_{i} w_{i} Y_{i}(t) e^{\boldsymbol{\beta}^{T} \mathbf{Z}_{i}} \mathbf{Z}_{i}^{\otimes 2} .
\end{gathered}
$$

Da mesma forma que no modelo usual, com esta notação, define-se a média ponderada $\overline{\mathbf{Z}}_{w}(\boldsymbol{\beta}, t)$ e a covariância empírica das covariáveis $\mathrm{V}_{w}(\boldsymbol{\beta}, t)$ como

$$
\overline{\mathbf{Z}}_{w}(\boldsymbol{\beta}, t)=\frac{S_{w}^{(1)}(\boldsymbol{\beta}, t)}{S_{w}^{(0)}(\boldsymbol{\beta}, t)}
$$




$$
\mathrm{V}_{w}(\boldsymbol{\beta}, t)=\frac{S_{w}^{(2)}(\boldsymbol{\beta}, t)}{S_{w}^{(0)}(\boldsymbol{\beta}, t)}-\left(\frac{S_{w}^{(1)}(\boldsymbol{\beta}, t)}{S_{w}^{(0)}(\boldsymbol{\beta}, t)}\right)^{\otimes 2}
$$

Portanto, a função escore e a matriz de informação do modelo de Cox ponderado podem ser escritas como

$$
\begin{gathered}
\mathbf{U}_{n, w}(\boldsymbol{\beta})=\sum_{i=1}^{n} R_{i} w_{i} \int_{0}^{\infty}\left\{\mathbf{Z}_{i}(s)-\overline{\mathbf{Z}}_{w}(\boldsymbol{\beta}, s)\right\} d N_{i}(s) \\
\mathbf{I}_{w}(\boldsymbol{\beta})=\sum_{i=1}^{n} R_{i} w_{i} \int_{0}^{\infty} \mathrm{V}_{w}(\boldsymbol{\beta}, s) d N_{i}(s) .
\end{gathered}
$$

Note que a função escore ponderada expressa em (B.27) não corresponde à soma ponderada dos termos da função escore usual, pois as ponderações também são incluídas nos termos $\mathbf{U}_{i}$ através de $\overline{\mathbf{Z}}_{w}$.

As propriedades assintóticas do estimador de máxima verossimilhança ponderado dependem se os pesos são conhecidos a priori ou se são estimados a partir dos dados. Pugh et al. (1993) utilizaram a teoria dos martingais para chegar na distribuição assintótica do estimador de máxima verossimilhança parcial ponderado, considerando como pesos o recíproco das probabilidades de dados completos.

Da mesma forma que no modelo de Cox não ponderado, a função escore ponderada pode ser expressa como um martingal

$$
\mathbf{U}_{n, w}(\boldsymbol{\beta})=\sum_{i=1}^{n} \int_{0}^{\infty} R_{i} w_{i}\left\{\mathbf{Z}_{i}(s)-\overline{\mathbf{Z}}_{w}(\boldsymbol{\beta}, s)\right\} d M_{i}(s)
$$


Para que $\mathbf{U}_{n, w}(\boldsymbol{\beta})$ seja um martingal de média zero, é preciso que as componentes da soma sejam martingais de média zero. Para isso, é necessário que a integral acima seja estocástica do tipo $\int_{0}^{t} H(s) d M(s)$ em que $H(t)$ é um processo previsível (Andersen et al. (1993)).

Quando os pesos $w_{i}$ são conhecidos, $\mathbf{U}_{n, w}$ corresponde a uma soma de martingais de média zero, pois $R_{i}, w_{i}$ e $\mathbf{Z}_{i}$ são fixos para qualquer $t$ e $Y_{i}(t)$ é contínuo à esquerda e, portanto, previsível. Logo, o processo escore é um martingal de média zero e usando o TLC para martingais, pode-se mostrar que $n^{-1 / 2} \mathbf{U}_{n, w}\left(\boldsymbol{\beta}_{0}\right)$ é assintoticamente normal.

Com a normalidade assintótica de $n^{-1 / 2} \mathbf{U}_{n, w}\left(\boldsymbol{\beta}_{0}\right)$ e o Teorema de Slutsky, Pugh et al. (1993) mostram que, para pesos conhecidos definidos por $w_{i}=R_{i} / \xi_{i} \operatorname{com} \xi_{i}$ expresso em (B.6) e tempos de falha limitados por $\tau$,

$$
n^{1 / 2}\left(\hat{\boldsymbol{\beta}}_{w}-\boldsymbol{\beta}_{0}\right) \stackrel{\mathcal{D}}{\longrightarrow} N\left(0, \boldsymbol{\Sigma}^{-1} \boldsymbol{\Sigma}_{w} \boldsymbol{\Sigma}^{-1}\right)
$$

em que $\boldsymbol{\Sigma}$ é a matriz de covariância assintótica do modelo não ponderado

$$
\boldsymbol{\Sigma}=\boldsymbol{\Sigma}\left(\boldsymbol{\beta}_{0}\right) \equiv-\mathrm{E}\left(\frac{\partial}{\partial \boldsymbol{\beta}} \int_{0}^{\tau}\left\{\mathbf{Z}_{i}-\mu\left(\boldsymbol{\beta}_{0}, s\right)\right\} d M_{i}(s)\right)
$$

e $\Sigma_{w}$ é a covariância assintótica do escore ponderado,

$$
\boldsymbol{\Sigma}_{w}=\boldsymbol{\Sigma}_{w}\left(\boldsymbol{\beta}_{0}\right) \equiv-\mathrm{E}\left(\frac{\partial}{\partial \boldsymbol{\beta}} \int_{0}^{\tau} \frac{R_{i}}{\xi_{i}}\left\{\mathbf{Z}_{i}-\mu\left(\boldsymbol{\beta}_{0}, s\right)\right\} d M_{i}(s)\right)
$$

$\operatorname{com} \mu(\boldsymbol{\beta}, t)=\lim _{n \rightarrow \infty} \overline{\mathbf{Z}}=\lim _{n \rightarrow \infty} \overline{\mathbf{Z}}_{w}$.

A matriz de covariância assintótica pode ser consistentemente estimada a partir dos dados, substituindo $\boldsymbol{\Sigma}$ e $\boldsymbol{\Sigma}_{w}$ por $\mathbf{I}\left(\hat{\boldsymbol{\beta}}_{w}\right)$ e $\mathbf{I}_{w}\left(\hat{\boldsymbol{\beta}}_{w}\right)$ dados pelas expressões (B.18) e (B.28), em que $\hat{\boldsymbol{\beta}}_{w}$ é o estimador de máxima verossimilhança parcial ponderado. 
É importante ressaltar que, neste caso, os pesos conhecidos correspondem a $R_{i} / \xi_{i}$, no qual $\xi_{i}=\mathrm{E}\left[R_{i} \mid \Delta_{i}, X_{i}, \mathbf{Z}_{i}\right]=\mathrm{E}\left[R_{i} \mid \Delta_{i}, X_{i}, \mathbf{Z}_{i, c o m p}\right]$. Além da consistência de $\hat{\boldsymbol{\beta}}$, para demonstrar a convergência em (4.30), algumas condições de regularidade devem estar satisfeitas, como que $\overline{\mathbf{Z}}_{w}(\boldsymbol{\beta}, u)$ e $\mathrm{V}_{w}(\boldsymbol{\beta}, u)$ convirjam uniformemente em $u$ para quantidades $\mu(\boldsymbol{\beta}, u)$ e $\mathbf{v}(\boldsymbol{\beta}, u)$. Para isso, é preciso assumir que existe um intervalo $[0, \tau]$ no qual a probabilidade de não omissão e a probabilidade de estar em risco sejam maiores que zero e que as covariáveis em $\mathbf{Z}_{i}$ sejam limitadas.

Na maioria das situações práticas, e também no contexto deste trabalho, os pesos não são conhecidos e precisam ser estimados por algum modelo. Neste caso, tem-se um modelo $\xi(\gamma)$, em que $\xi$ pode ser a probabilidade de dado completo ou a probabilidade de cura. Desta forma, a função escore ponderada depende também da parametrização dos pesos e é dada por

$$
\mathbf{U}_{n, w}(\boldsymbol{\beta}, \boldsymbol{\gamma})=\sum_{i=1}^{n} \int_{0}^{\infty} \frac{R_{i}}{\xi_{i}(\boldsymbol{\gamma})}\left\{\mathbf{Z}_{i}-\overline{\mathbf{Z}}_{w}(\boldsymbol{\beta}, \boldsymbol{\gamma}, s)\right\} d M_{i}(s)
$$

$\operatorname{com} \overline{\mathbf{Z}}_{w}(\boldsymbol{\beta}, \boldsymbol{\gamma}, t)=\frac{\sum_{j=1}^{n} \frac{R_{j}}{\xi_{j}(\gamma)} Y_{j}(t) \exp \left\{\beta^{\prime} Z_{j}\right\} Z_{j}}{\sum_{j=1}^{n} \frac{R_{j}}{\xi_{j}(\gamma)} Y_{j}(t) \exp \left\{\beta^{\prime} Z_{j}\right\}}$.

Note que $\mathbf{U}_{n, w}(\boldsymbol{\beta}, \boldsymbol{\gamma})$ continua sendo um martingal de média zero pois, como nos dois tipos de ponderação, $\xi(\gamma)$ não depende de t, o processo pontual no integrando continua sendo previsível.

Com este resultado, Pugh et al. (1993) mostram a consistência e normalidade assintótica do estimador de máxima verossimilhança ponderado por estimativas dos pesos logísticos. A proposta é inicialmente ajustar um modelo de regressão logística usual para obter o estimador de máxima verossimilhança de $\boldsymbol{\gamma}$ e, em seguida, estimar $\boldsymbol{\beta}$ solucionando $\mathbf{U}_{n, w}(\boldsymbol{\beta}, \hat{\gamma})=0$, assumindo que $\hat{\boldsymbol{\gamma}}$ é um parâmetro fixo. Assim como no modelo não ponderado, usando expansão de Taylor e o teorema limite central para 
martingais, chega-se na distribuição assintótica do estimador proposto.

A função escore do modelo usual de regressão logística é dada por

$$
\mathbf{T}_{n}(\boldsymbol{\gamma})=\sum_{i=1}^{n} \frac{\partial}{\partial \boldsymbol{\gamma}}\left[R_{i} \log \xi_{i}(\boldsymbol{\gamma})+\left(1-R_{i}\right) \log \left(1-\xi_{i}(\boldsymbol{\gamma})\right)\right]
$$

e o estimador de máxima verossimilhança de $\gamma$ é obtido por $\mathbf{T}_{n}(\gamma)=0$. Considerando $\hat{\boldsymbol{\gamma}}$ conhecido e igual ao verdadeiro valor $\boldsymbol{\gamma}_{0}$, resolve-se a equação $\mathbf{U}_{n, w}(\boldsymbol{\beta}, \hat{\gamma})=0$ para obter o estimador ponderado $\hat{\boldsymbol{\beta}}_{w}(\hat{\gamma})$.

A distribuição assintótica de $\hat{\boldsymbol{\beta}}_{w}(\hat{\gamma})$ é obtida a partir da expansão de Taylor de $n^{-1 / 2} \mathbf{U}_{n, w}(\boldsymbol{\beta}, \boldsymbol{\gamma})$ em torno de $\left(\boldsymbol{\beta}_{0}, \boldsymbol{\gamma}_{0}\right)$. A normalidade segue do fato de que as funções escore $n^{-1 / 2} \mathbf{T}_{n}\left(\boldsymbol{\gamma}_{0}\right)$ e $n^{-1 / 2} \mathbf{U}_{n, w}\left(\boldsymbol{\beta}_{0}, \boldsymbol{\gamma}_{0}\right)$ são assintoticamente equivalentes a $n^{-1 / 2}$ vezes as somas dos termos i.i.d

$$
\mathbf{T}_{i}=\mathbf{T}_{n, i}\left(\boldsymbol{\gamma}_{0}\right)=\left.\frac{\partial}{\partial \boldsymbol{\gamma}}\left[R_{i} \log \xi_{i}(\boldsymbol{\gamma})+\left(1-R_{i}\right) \log \left(1-\xi_{i}(\boldsymbol{\gamma})\right)\right]\right|_{\boldsymbol{\gamma}=\boldsymbol{\gamma}_{0}}
$$

e

$$
\mathbf{U}_{i}=\mathbf{U}_{n, w, i}^{\mu}\left(\boldsymbol{\beta}_{0}, \boldsymbol{\gamma}_{0}\right) \equiv \int_{0}^{\tau} \frac{R_{i}}{\xi_{i}\left(\boldsymbol{\gamma}_{0}\right)}\left\{\mathbf{Z}_{i}-\mu\left(\boldsymbol{\beta}_{0}, s\right)\right\} d M_{i}(s)
$$

em que $\boldsymbol{\beta}_{0}$ e $\boldsymbol{\gamma}_{0}$ são os verdadeiros valores dos parâmetros $\boldsymbol{\beta}$ e $\boldsymbol{\gamma}$.

Pugh et al. (1993) mostram que, sob certas condições de regularidade,

$$
n^{1 / 2}\left(\hat{\boldsymbol{\beta}}(\hat{\boldsymbol{\gamma}})-\boldsymbol{\beta}_{0}\right) \stackrel{\mathcal{D}}{\longrightarrow} N\left(\mathbf{0}, \quad \boldsymbol{\Sigma}_{w}^{-1} \mathrm{~V}^{*} \boldsymbol{\Sigma}_{w}^{-1}\right)
$$

em que $\boldsymbol{\Sigma}_{w} \equiv-\mathrm{E}\left[\frac{\partial}{\partial \boldsymbol{\beta}} \mathbf{U}_{i}\right]$, e $\mathrm{V}^{*} \equiv E\left[\mathbf{U}_{i} \mathbf{U}_{i}^{\prime}\right]-E\left[\mathbf{U}_{i} \mathbf{T}_{i}^{\prime}\right] E\left[\mathbf{T}_{i} \mathbf{T}_{i}^{\prime}\right]^{-1} E\left[\mathbf{T}_{i} \mathbf{U}_{i}^{\prime}\right]$. 
A seção B.2.3. traz alguns passos utilizados para demonstrar o resultado (B.37).

A matriz $\boldsymbol{\Sigma}_{w}$ pode ser consistentemente estimada por $\mathbf{I}_{w}(\hat{\boldsymbol{\beta}}(\hat{\boldsymbol{\gamma}}))$ dada em (B.28) substituindo $w_{i}$ por $1 / \hat{\xi}_{i}=1 / \xi_{i}(\hat{\gamma})$. Segundo Pugh et al. (1993), uma estimativa de $\mathrm{V}^{*}$ pode ser obtida pela matriz de covariância empírica dos resíduos de uma regressão de $\hat{\mathbf{U}}_{i}$ em $\hat{\mathbf{T}}_{i}$, em que

$$
\hat{\mathbf{T}}_{i}=\left.\frac{\partial}{\partial \boldsymbol{\gamma}}\left[R_{i} \log \xi_{i}(\boldsymbol{\gamma})+\left(1-R_{i}\right) \log \left(1-\xi_{i}(\boldsymbol{\gamma})\right)\right]\right|_{\boldsymbol{\gamma}=\hat{\boldsymbol{\gamma}}}
$$

$\mathrm{e}$

$$
\hat{\mathbf{U}}_{i}=\int_{0}^{\tau} \frac{R_{i}}{\xi_{i}(\hat{\gamma})}\left\{\mathbf{Z}_{i}-\frac{\mathbf{S}_{w}^{(1)}(\hat{\boldsymbol{\beta}}(\hat{\gamma}), s)}{\mathbf{S}_{w}^{(0)}(\hat{\boldsymbol{\beta}}(\hat{\gamma}), s)}\right\} d \hat{M}_{i}(s)
$$

Pela definição do processo $M_{i}$, tem-se que $d M_{i}(s)=d N_{i}(s)-h_{0}(s) Y_{i}(s) \exp ^{\boldsymbol{\beta}_{0}^{\prime} \mathbf{Z}_{i}} d s$. Logo, para estimar $\mathbf{U}_{i}$ ainda é preciso obter uma estimativa da função de risco padrão $h_{0}(s)$. Pugh et al. (1993) sugerem um estimador de Breslow ponderado

$$
\hat{H}_{0}(t)=\int_{0}^{t} \frac{\sum_{j=1}^{n} d N_{j}(s)}{\sum_{j=1}^{n} \frac{R_{j}}{\xi_{i}(\hat{\gamma})} Y_{j}(s) \exp ^{\boldsymbol{\beta}(\hat{\gamma})^{\prime} \mathbf{Z}_{j}}}
$$

que resulta em

$$
\hat{h}_{0}(t)=\frac{\sum_{j=1}^{n} d N_{j}(s)}{\sum_{j=1}^{n} \frac{R_{j}}{\xi_{i}(\hat{\gamma})} Y_{j}(s) \exp ^{\boldsymbol{\beta}(\hat{\gamma})^{\prime} \mathbf{Z}_{j}}} .
$$

As condições de regularidade para mostrar a distribuição assintótica do estimador com pesos logísticos consistem em assumir que o vetor de covariáveis $\mathbf{Z}$ é constante no tempo e limitado, que os tempos de falha e censura são condicionalmente independentes 
dado $\mathbf{Z}$, que a matriz de covariância assintótica é positiva definida e que o eixo do tempo está restrito ao intervalo $[0, \tau]$ no qual a probabilidade de estar em risco e a probabilidade de ter dados completos são limitadas inferiormente por $\delta$, tal que $\delta>0$.

Embora os processos de estimação dos pesos logísticos e dos pesos FC sejam diferentes, ambos correspondem a estimadores de máxima verossimilhança de um modelo de regressão logística usual. A diferença é que para os pesos logísticos, a resposta é o indicador de dados completos $R_{i}$ e para os pesos FC a resposta é o indicador de não cura $b_{i}$. Apesar disso, a teoria desenvolvida para pesos logísticos não se aplica diretamente aos pesos FC. Mesmo para pesos conhecidos e satisfeitas as suposições de que a probabilidade de cura $\left(\xi_{i}\right)$ não se encontra na fronteira do espaço paramétrico e depende apenas dos tempos de falha ou censura e das covariáveis completamente observadas, temos $\mathrm{E}\left(R_{i} / \xi_{i} \mid \Delta_{i}, X_{i}, \mathbf{Z}_{i}\right) \neq 1$, a não ser que $R_{i}=1-b_{i}, \quad \forall i=1, \ldots, n$, isto é, que todos os indivíduos com dados completos sejam curados ou, equivalentemente, que a omissão implique diretamente em não cura.

\section{B.2.3 Distribuição assintótica do estimador ponderado no mo- delo de Cox}

A seguir são apresentados alguns detalhes do desenvolvimento de Pugh et al. (1993) para mostrar a convergência dada em (B.37).

O primeiro passo para provar a distribuição assintótica do estimador ponderado é mostrar que a função escore ponderada é assintoticamente não viciada, isto é, que $n^{-1} \mathbf{U}_{n, w}(\boldsymbol{\beta})$ converge quase certamente para uma função $\mathbf{U}(\boldsymbol{\beta})$ tal que $\mathbf{U}\left(\boldsymbol{\beta}_{0}\right)=\mathbf{0}$. Esta propriedade vem da equivalência assintótica entre a função escore ponderada e a função escore não ponderada.

Sejam $\left(\Delta_{i}, X_{i}, \mathbf{Z}_{\mathbf{i}}, R_{i}\right)$ variáveis aleatórias independentes identicamente distribuídas. Usando a notação em (4.21) e o fato de que $\mathrm{E}\left(\frac{R_{1}}{\xi_{1}} \mid \Delta_{1}, X_{1}, \mathbf{Z}_{1}\right)=1$, tem-se que 


$$
\begin{aligned}
\mathrm{E}\left(S_{w}^{(0)}(\boldsymbol{\beta}, u)\right) & =\mathrm{E}\left\{\frac{R_{1}}{\xi_{1}} Y_{1}(u) \mathrm{e}^{\boldsymbol{\beta}^{\prime} \mathbf{Z}_{1}}\right\} \\
& =\mathrm{E}\left\{\mathrm{E}\left(\frac{R_{1}}{\xi_{1}} Y_{1}(u) \mathrm{e}^{\boldsymbol{\beta}^{\prime} \mathbf{Z}_{1}} \mid \Delta_{1}, X_{1}, \mathbf{Z}_{1}\right)\right\} \\
& =\mathrm{E}\left\{\mathrm{e}^{\boldsymbol{\beta}^{\prime} \mathbf{Z}_{1}} P\left(Y_{1}(u)=1 \mid \mathbf{Z}_{1}\right)\right\}
\end{aligned}
$$

Defina $s^{(0)}(\boldsymbol{\beta}, u)=\mathrm{E}\left(S_{w}^{(0)}(\boldsymbol{\beta}, u)\right)$. Assumindo que $\mathrm{E}\left(\mathrm{e}^{\boldsymbol{\beta}^{\prime} \mathbf{Z}_{1}}\right)<\infty$, tem-se que $\mathrm{E}\left\{Y_{1}(u) e^{\boldsymbol{\beta}^{\prime} \mathbf{Z}_{\mathbf{1}}}\right\} \leq \mathrm{E}\left\{e^{\boldsymbol{\beta}^{\prime} \mathbf{Z}_{\mathbf{1}}}\right\}$, o que implica que $s^{(0)}(\boldsymbol{\beta}, u)$ é finita. Aplicando a lei forte dos grandes números de Kolmogorov, segue que $S_{w}^{(0)}(\boldsymbol{\beta}, u) \stackrel{q . c .}{\longrightarrow} s^{(0)}(\boldsymbol{\beta}, u)$. Analogamente, pode-se mostrar que $S_{w}^{(1)}(\boldsymbol{\beta}, u) \stackrel{\text { q.c. }}{\longrightarrow} s^{(1)}(\boldsymbol{\beta}, u)$ e $S_{w}^{(2)}(\boldsymbol{\beta}, u) \stackrel{\text { q.c. }}{\longrightarrow} s^{(2)}(\boldsymbol{\beta}, u)$.

Note que $\overline{\mathbf{Z}}_{w}(\boldsymbol{\beta}, u)=\frac{S^{(0)}(\boldsymbol{\beta}, u)}{S^{(1)}(\boldsymbol{\beta}, u)}$ é uma razão de médias ponderadas de variáveis aleatórias limitadas e identicamente distribuídas. Logo, aplicando a lei forte dos grandes números ao numerador e denominador de $\overline{\mathbf{Z}}_{w}(\boldsymbol{\beta}, u)$, obtem-se a convergência pontual de $\overline{\mathbf{Z}}_{w}$ para $\mu$.

A partir da convergência pontual, Pugh et al. (1993) modificam o Teorema de Glivenko Cantelli (Sen \& Singer 1993) para mostrar a convergência uniforme em $u$, isto é,

$$
\sup _{0 \leq u \leq \tau}\left|\overline{\mathbf{Z}}_{w}(\boldsymbol{\beta}, u)-\mu(\boldsymbol{\beta}, u)\right| \stackrel{\text { q.c. }}{\longrightarrow} 0
$$

em que $\mu(\boldsymbol{\beta}, t)=\lim _{n \rightarrow \infty} \overline{\mathbf{Z}}_{w}(\boldsymbol{\beta}, t)=\frac{s^{(1)}(\boldsymbol{\beta}, t)}{s^{(0)}(\boldsymbol{\beta}, t)}$

Este resultado implica a equivalência assintótica entre $\mathbf{U}_{n, w}\left(\boldsymbol{\beta}_{0}, \boldsymbol{\gamma}_{0}\right)$ e $\mathbf{U}_{n, w}^{\mu}\left(\boldsymbol{\beta}_{0}, \boldsymbol{\gamma}_{0}\right)$, que corresponde a $\mathbf{U}_{n, w}$ substituindo a média empírica $\overline{\mathbf{Z}}_{w}$ por seu limite assintótico $\mu=\lim _{n \rightarrow \infty} \overline{\mathbf{Z}}_{w}$. Note que o termo $\overline{\mathbf{Z}}$ presente na função escore não ponderada pode ser considerado um caso particular de $\overline{\mathbf{Z}}_{w}$ com todos os pesos iguais a 1. Logo, este resultado também é suficiente para mostrar a equivalência assintótica entre $\mathbf{U}_{n}\left(\boldsymbol{\beta}_{0}, \boldsymbol{\gamma}_{0}\right)$ e $\mathbf{U}_{n}^{\mu}\left(\boldsymbol{\beta}_{0}, \boldsymbol{\gamma}_{0}\right)$. 
O mesmo procedimento pode ser usado para provar que $\mathrm{V}_{w}(\boldsymbol{\beta}, u, u)$ converge para $\mathbf{v}(\boldsymbol{\beta}, u)=\frac{s^{(2)}(\boldsymbol{\beta}, u)}{s^{(0)}(\boldsymbol{\beta}, u)}-\left(\frac{s^{(1)}(\boldsymbol{\beta}, u)}{s^{(0)}(\boldsymbol{\beta}, u)}\right)^{\otimes 2}$, uniformemente em $u$. Com este resultado, pode-se mostrar que $\frac{\partial}{\partial \boldsymbol{\beta}} \mathbf{U}_{n, w}(\boldsymbol{\beta}, \tau)$ converge para $-\int_{0}^{\tau} \mathbf{v}(\boldsymbol{\beta}, s) d N_{i}(s)$, quando $n \rightarrow \infty$.

O passo seguinte é demonstrar a normalidade assintótica de $n^{-1 / 2} \mathbf{U}_{n, w}\left(\boldsymbol{\beta}_{0}, \boldsymbol{\gamma}_{0}\right)$. Como $\mathbf{U}_{n, w}$ e $\mathbf{U}_{n, w}^{\mu}$ são equivalentes, pode-se mostrar a normalidade de $n^{-1 / 2} \mathbf{U}_{n, w}^{\mu}\left(\boldsymbol{\beta}_{0}, \boldsymbol{\gamma}_{0}\right)$.

Para simplificar a notação, as componentes da soma do processo $\mathbf{U}_{n, w}^{\mu}\left(\boldsymbol{\beta}_{0}, \boldsymbol{\gamma}_{0}, \tau\right)$ serão denotadas por $\mathbf{U}_{i}$, isto é, $\mathbf{U}_{i}=\int_{0}^{\tau} \frac{R_{i}}{\xi_{i}\left(\gamma_{0}\right)}\left\{\mathbf{Z}_{i}-\mu\left(\boldsymbol{\beta}_{0}, s\right)\right\} d N_{i}(s)$.

Note que

$$
\begin{aligned}
\mathrm{E}\left(\mathbf{U}_{i}\right) & =\mathrm{E}\left\{\int_{0}^{\tau} \frac{R_{i}}{\xi_{i}\left(\boldsymbol{\gamma}_{0}\right)}\left\{\mathbf{Z}_{i}-\mu\left(\boldsymbol{\beta}_{0}, s\right)\right\} d M_{i}(s)\right\} \\
& =\mathrm{E}\left\{\int_{0}^{\tau}\left\{\mathbf{Z}_{i}-\mu\left(\boldsymbol{\beta}_{0}, s\right)\right\} d M_{i}(s) \mathrm{E}\left\{\frac{R_{i}}{\xi_{i}\left(\boldsymbol{\gamma}_{0}\right)} \mid \Delta_{i}, X_{i}, \mathbf{Z}_{\mathbf{i}}\right\}\right\} \\
& =\mathrm{E}\left\{\int_{0}^{\tau}\left\{\mathbf{Z}_{i}-\mu\left(\boldsymbol{\beta}_{0}, s\right)\right\} d M_{i}(s)\right\}=\mathbf{0},
\end{aligned}
$$

pois $M_{i}(s)$ é um martingal de média zero e $\mathbf{Z}_{i}-\mu\left(\boldsymbol{\beta}_{0}, s\right)$ é previsível.

Com os mesmos argumentos, verifica-se que

$$
\begin{aligned}
\boldsymbol{\Sigma}_{w}=\mathrm{E}\left(\mathbf{U}_{i} \mathbf{U}_{i}^{\prime}\right) & =\mathrm{E}\left\{\left\{\int_{0}^{\tau} \frac{R_{i}}{\xi_{i}\left(\boldsymbol{\gamma}_{0}\right)}\left\{\mathbf{Z}_{i}-\mu\left(\boldsymbol{\beta}_{0}, s\right)\right\} d M_{i}(s)\right\}^{\otimes 2}\right\} \\
& =\mathrm{E}\left\{\frac{1}{\xi_{i}\left(\boldsymbol{\gamma}_{0}\right)} \int_{0}^{\tau}\left\{\left(\mathbf{Z}_{i}-\mu\left(\boldsymbol{\beta}_{0}, s\right)\right) d M_{i}(s)\right\}^{\otimes 2}\right\} .
\end{aligned}
$$

Sob a suposição que $\xi_{i} \geq \delta^{2}>0$, aplicando a desigualdade de Cauchy-Schwarz e resultados para o processo de variação quadrática de martingais, pode-se mostrar que os elementos de $\boldsymbol{\Sigma}_{w}$ são finitos. Logo, as componentes $\mathbf{U}_{\mathbf{i}}$ são vetores aleatórios i.i.d, com média zero e variância finita e a normalidade segue do teorema limite central. 
Da mesma forma que na teoria clássica de verossimilhança, a distribuição assintótica de $\hat{\boldsymbol{\beta}}_{w}(\hat{\gamma})$ é obtida a partir de uma expansão de Taylor do escore ponderado em torno dos verdadeiros valores dos parâmetros.

Expandindo $\mathbf{U}_{n, w}(\hat{\boldsymbol{\beta}}(\hat{\gamma}), \hat{\gamma})$ em torno de $\left(\boldsymbol{\beta}_{0}, \boldsymbol{\gamma}_{0}\right)$, tem-se que

$$
\begin{aligned}
0 & =\mathbf{U}_{n, w}(\hat{\boldsymbol{\beta}}(\hat{\boldsymbol{\gamma}}), \hat{\boldsymbol{\gamma}}) \\
& \approx \mathbf{U}_{n, w}\left(\boldsymbol{\beta}_{0}, \boldsymbol{\gamma}_{0}\right)+\frac{\partial}{\partial \boldsymbol{\beta}} \mathbf{U}_{n, w}\left(\boldsymbol{\beta}_{0}, \boldsymbol{\gamma}_{0}\right)\left(\hat{\boldsymbol{\beta}}(\hat{\boldsymbol{\gamma}})-\boldsymbol{\beta}_{0}\right)+\frac{\partial}{\partial \boldsymbol{\gamma}} \mathbf{U}_{n, w}\left(\boldsymbol{\beta}_{0}, \boldsymbol{\gamma}_{0}\right)\left(\hat{\boldsymbol{\gamma}}-\boldsymbol{\gamma}_{0}\right)
\end{aligned}
$$

Com o objetivo de obter uma aproximação para $n^{1 / 2}\left(\hat{\boldsymbol{\beta}}(\hat{\gamma})-\boldsymbol{\beta}_{0}\right)$, será analisado cada termo da expressão (B.46).

Verificou-se anteriormente que

$$
n^{-1 / 2} \mathbf{U}_{n, w}\left(\boldsymbol{\beta}_{0}, \boldsymbol{\gamma}_{0}\right) \approx n^{-1 / 2} \mathbf{U}_{n, w}^{\mu}\left(\boldsymbol{\beta}_{0}, \boldsymbol{\gamma}_{0}\right)=n^{-1 / 2} \sum_{i=1}^{n} \mathbf{U}_{i}\left(\boldsymbol{\beta}_{0}, \boldsymbol{\gamma}_{0}\right)
$$

considerando a notação $\mathbf{U}_{i}=\mathbf{U}_{w, i}^{\mu}$.

Conseqüentemente,

$$
n^{-1} \frac{\partial}{\partial \boldsymbol{\beta}} \mathbf{U}_{n, w}\left(\boldsymbol{\beta}_{0}, \boldsymbol{\gamma}_{0}\right) \stackrel{q . c .}{\longrightarrow} \mathrm{E}\left(\frac{\partial}{\partial \boldsymbol{\beta}} \mathbf{U}_{w, i}\left(\boldsymbol{\beta}_{0}, \boldsymbol{\gamma}_{0}\right)\right) \approx \mathrm{E}\left\{\frac{\partial}{\partial \boldsymbol{\beta}} \mathbf{U}_{i}\left(\boldsymbol{\beta}_{0}, \boldsymbol{\gamma}_{0}\right)\right\}
$$

e

$$
n^{-1} \frac{\partial}{\partial \boldsymbol{\gamma}} \mathbf{U}_{n, w}\left(\boldsymbol{\beta}_{0}, \boldsymbol{\gamma}_{0}\right) \stackrel{\text { q.c. }}{\longrightarrow} \mathrm{E}\left(\frac{\partial}{\partial \boldsymbol{\gamma}} \mathbf{U}_{w, i}\left(\boldsymbol{\beta}_{0}, \boldsymbol{\gamma}_{0}\right)\right) \approx \mathrm{E}\left\{\frac{\partial}{\partial \boldsymbol{\gamma}} \mathbf{U}_{i}\left(\boldsymbol{\beta}_{0}, \boldsymbol{\gamma}_{0}\right)\right\}
$$

Para expressar mais convenientemente o termo em (B.49), considere o vetor aleatório 
$\mathbf{D}_{i}=\left(R_{i}, X_{i}, \Delta_{i}, \mathbf{Z}_{\mathbf{i}}\right)$ e sejam $f_{\mathbf{D}}$ e $F_{\mathbf{D}}$ suas respectivas funções densidade e distribuição.

Como a esperança da função escore é zero, pode-se escrever

$$
\begin{aligned}
\mathbf{0} & =\frac{\partial}{\partial \boldsymbol{\gamma}} \mathrm{E}\left[\mathbf{U}_{i}(\boldsymbol{\beta}, \boldsymbol{\gamma})\right] \\
& =\frac{\partial}{\partial \boldsymbol{\gamma}} \int \mathbf{U}_{i}(\boldsymbol{\beta}, \boldsymbol{\gamma}, s) f_{\mathbf{D}}(\boldsymbol{\beta}, \boldsymbol{\gamma}, s) d F_{\mathbf{D}}(s) \\
& =\int\left\{\frac{\partial}{\partial \boldsymbol{\gamma}} \mathbf{U}_{i}(\boldsymbol{\beta}, \boldsymbol{\gamma}, s)\right\} f_{\mathbf{D}}(\boldsymbol{\beta}, \boldsymbol{\gamma}, s) d F_{\mathbf{D}}(s)+\int \mathbf{U}_{i}(\boldsymbol{\beta}, \boldsymbol{\gamma}, s) \frac{\partial}{\partial \boldsymbol{\gamma}, s} f_{\mathbf{D}}(\boldsymbol{\beta}, \boldsymbol{\gamma}, s) d F_{\mathbf{D}}(s) \\
& =\int\left\{\frac{\partial}{\partial \boldsymbol{\gamma}} \mathbf{U}_{i}(\boldsymbol{\beta}, \boldsymbol{\gamma}, s)\right\} f_{\mathbf{D}}(\boldsymbol{\beta}, \boldsymbol{\gamma}, s) d F_{\mathbf{D}}(s)+\int \mathbf{U}_{i}(\boldsymbol{\beta}, \boldsymbol{\gamma}, s) \frac{\frac{\partial}{\partial \boldsymbol{\gamma}} f_{\mathbf{D}}(\boldsymbol{\beta}, \boldsymbol{\gamma}, s)}{f_{\mathbf{D}}(\boldsymbol{\beta}, \boldsymbol{\gamma}, s)} f_{\mathbf{D}}(\boldsymbol{\beta}, \boldsymbol{\gamma}, s) d F_{\mathbf{D}}(s) \\
& =\mathrm{E}\left\{\frac{\partial}{\partial \boldsymbol{\gamma}} \mathbf{U}_{i}(\boldsymbol{\beta}, \boldsymbol{\gamma})\right\}+\mathrm{E}\left\{\mathbf{U}_{i}(\boldsymbol{\beta}, \boldsymbol{\gamma}) \frac{\partial}{\partial \boldsymbol{\gamma}} \log f_{\mathbf{D}}(\boldsymbol{\beta}, \boldsymbol{\gamma})\right\} .
\end{aligned}
$$

Assumindo que os dois vetores de parâmetros são independentes, isto é, que a distribuição do indicador de omissão depende de $\boldsymbol{\gamma}$ e não de $\boldsymbol{\beta}$ e que a distribuição dos tempos de sobrevivência depende de $\boldsymbol{\beta}$ e não de $\boldsymbol{\gamma}$, a quantidade $\frac{\partial}{\partial \boldsymbol{\gamma}} \log f_{\mathbf{D}}(\boldsymbol{\beta}, \boldsymbol{\gamma})$ pode ser simplificada.

Neste caso

$$
\frac{\partial}{\partial \boldsymbol{\gamma}} \log f_{\mathbf{D}}(\boldsymbol{\beta}, \boldsymbol{\gamma})=\frac{\partial}{\partial \boldsymbol{\gamma}}\left\{\log f_{\mathbf{D}}\left(R_{i} / X_{i}, \Delta_{i}, \mathbf{Z}_{i}, \boldsymbol{\gamma}\right)+\log f_{\mathbf{D}}\left(X_{i}, \Delta_{i}, \mathbf{Z}_{i}, \boldsymbol{\beta}\right)\right\}
$$

Sob a suposição que a omissão depende apenas das covariáveis completamente observadas,

$$
\frac{\partial}{\partial \boldsymbol{\gamma}} \log f_{\mathbf{D}}(\boldsymbol{\beta}, \boldsymbol{\gamma})=\frac{\partial}{\partial \boldsymbol{\gamma}} \log f_{\mathbf{D}}\left(R_{i} / X_{i}, \Delta_{i}, \mathbf{Z}_{i, c o m p}, \boldsymbol{\gamma}\right)=\mathbf{T}_{i}(\boldsymbol{\gamma})
$$

Portanto, substituindo o resultado acima em (B.50), tem-se que 


$$
\begin{aligned}
\mathbf{0}=\frac{\partial}{\partial \boldsymbol{\gamma}} \mathrm{E}\left[\mathbf{U}_{i}(\boldsymbol{\beta}, \boldsymbol{\gamma})\right] & =\mathrm{E}\left\{\frac{\partial}{\partial \boldsymbol{\gamma}} \mathbf{U}_{i}(\boldsymbol{\beta}, \boldsymbol{\gamma})\right\}+\mathrm{E}\left\{\mathbf{U}_{i}(\boldsymbol{\beta}, \boldsymbol{\gamma}) \mathbf{T}_{i}^{\prime}(\boldsymbol{\gamma})\right\} \Rightarrow \\
\mathrm{E}\left\{\frac{\partial}{\partial \boldsymbol{\gamma}} \mathbf{U}_{i}(\boldsymbol{\beta}, \boldsymbol{\gamma})\right\} & =-\mathrm{E}\left\{\mathbf{U}_{i}(\boldsymbol{\beta}, \boldsymbol{\gamma}) \mathbf{T}_{i}^{\prime}(\boldsymbol{\gamma})\right\}
\end{aligned}
$$

Usando este resultado, a expressão (B.49) pode ser escrita como

$$
n^{-1} \frac{\partial}{\partial \boldsymbol{\gamma}} \mathbf{U}_{n, w}\left(\boldsymbol{\beta}_{0}, \boldsymbol{\gamma}_{0}\right) \approx-\mathrm{E}\left\{\mathbf{U}_{i}\left(\boldsymbol{\beta}_{0}, \boldsymbol{\gamma}_{0}\right) \mathbf{T}_{i}^{\prime}\left(\boldsymbol{\gamma}_{0}\right)\right\}
$$

O termo $\left(\hat{\gamma}-\gamma_{0}\right)$ em (B.46) pode ser aproximado via expansão de Taylor da função escore $\mathbf{T}_{n}(\boldsymbol{\gamma})$. Expandindo $\mathbf{T}_{n}(\hat{\gamma})$ em torno de $\boldsymbol{\gamma}_{0}$ tem-se que

$$
\begin{aligned}
0=n^{-1 / 2} \mathbf{T}_{n}(\hat{\gamma}) & \approx n^{-1 / 2} \mathbf{T}_{n}\left(\boldsymbol{\gamma}_{0}\right)+n^{-1 / 2} \frac{\partial}{\partial \boldsymbol{\gamma}} \mathbf{T}_{n}\left(\boldsymbol{\gamma}_{0}\right)\left(\hat{\gamma}-\boldsymbol{\gamma}_{0}\right) \Rightarrow \\
n^{1 / 2}\left(\hat{\gamma}-\boldsymbol{\gamma}_{0}\right) & \approx-n^{-1 / 2} \mathbf{T}_{n}\left(\boldsymbol{\gamma}_{0}\right)\left\{n^{-1} \frac{\partial}{\partial \boldsymbol{\gamma}} \mathbf{T}_{n}\left(\boldsymbol{\gamma}_{0}\right)\right\}^{-1}
\end{aligned}
$$

Mas, sob a teoria clássica de verossimilhança

$$
n^{-1} \frac{\partial}{\partial \boldsymbol{\gamma}} \mathbf{T}_{n}\left(\boldsymbol{\gamma}_{0}\right) \stackrel{\text { q.c. }}{\longrightarrow} \mathrm{E}\left(\frac{\partial}{\partial \boldsymbol{\gamma}} \mathbf{T}_{i}\left(\boldsymbol{\gamma}_{0}\right)\right)=-E\left[\mathbf{T}_{i}\left(\boldsymbol{\gamma}_{0}\right) \mathbf{T}_{i}^{\prime}\left(\boldsymbol{\gamma}_{0}\right)\right]
$$

Logo,

$$
n^{1 / 2}\left(\hat{\gamma}-\gamma_{0}\right) \approx n^{-1 / 2} \mathbf{T}_{n}\left(\boldsymbol{\gamma}_{0}\right)\left\{E\left[\mathbf{T}_{i}\left(\boldsymbol{\gamma}_{0}\right) \mathbf{T}_{i}^{\prime}\left(\boldsymbol{\gamma}_{0}\right)\right]\right\}^{-1}
$$

Substituindo (B.47), (B.48), (B.52) e (B.55) em (B.46) e multiplicando por $n^{-1 / 2}$ obtém-se 


$$
\begin{aligned}
0 & n^{-1 / 2} \sum_{i=1}^{n} \mathbf{U}_{i}\left(\boldsymbol{\beta}_{0}, \boldsymbol{\gamma}_{0}\right)+n^{1 / 2}\left(\hat{\boldsymbol{\beta}}(\hat{\boldsymbol{\gamma}})-\boldsymbol{\beta}_{0}\right) \mathrm{E}\left(\frac{\partial}{\partial \boldsymbol{\beta}} \mathbf{U}_{i}\left(\boldsymbol{\beta}_{0}, \boldsymbol{\gamma}_{0}\right)\right) \\
- & \mathrm{E}\left(\mathbf{U}_{i}\left(\boldsymbol{\beta}_{0}, \boldsymbol{\gamma}_{0}\right) \mathbf{T}_{i}^{\prime}\left(\boldsymbol{\gamma}_{0}\right)\right) n^{-1 / 2} \mathbf{T}_{n}\left(\boldsymbol{\gamma}_{0}\right)\left(\mathrm{E}\left(\mathbf{T}_{i}\left(\boldsymbol{\gamma}_{0}\right) \mathbf{T}_{i}^{\prime}\left(\boldsymbol{\gamma}_{0}\right)\right)\right)^{-1} \Rightarrow \\
\Rightarrow & n^{1 / 2}\left(\hat{\boldsymbol{\beta}}(\hat{\boldsymbol{\gamma}})-\boldsymbol{\beta}_{0}\right) \approx\left\{\mathrm{E}\left(-\frac{\partial}{\partial \boldsymbol{\beta}} \mathbf{U}_{i}\left(\boldsymbol{\beta}_{0}, \boldsymbol{\gamma}_{0}\right)\right)\right\}^{-1} \times \\
& n^{-1 / 2} \sum_{i=1}^{n}\left\{\mathbf{U}_{i}\left(\boldsymbol{\beta}_{0}, \boldsymbol{\gamma}_{0}\right)-\mathrm{E}\left(\mathbf{U}_{i}\left(\boldsymbol{\beta}_{0}, \boldsymbol{\gamma}_{0}\right) \mathbf{T}_{i}^{\prime}\left(\boldsymbol{\gamma}_{0}\right)\right)\left(\mathrm{E}\left(\mathbf{T}_{i}\left(\boldsymbol{\gamma}_{0}\right) \mathbf{T}_{i}^{\prime}\left(\boldsymbol{\gamma}_{0}\right)\right)\right)^{-1} \mathbf{T}_{i}\left(\boldsymbol{\gamma}_{0}\right)\right\}
\end{aligned}
$$

A normalidade assintótica segue do Teorema do Limite Central. 


\section{Apêndice C}

\section{Aspectos Computacionais}

Para o caso usual sem ponderação, os modelos com fração de cura semiparamétricos podem ser ajustados utilizando a rotina em S-Plus chamada semicure escrita por Peng (2003b). Para o modelo com fração de cura Weibull, pode ser utilizada a rotina gfcure em R desenvolvida por Peng et al. (1998) para a distribuição F generalizada (Kalbfleisch \& Prentice (2002)). Ambas as rotinas estão disponíveis em www.math.mun.ca/ ypeng/research.

Na metodologia proposta, a estimação dos pesos é realizada independentemente do ajuste dos modelos finais ponderados. Para o método de pesos logísticos utilizou-se a função $\mathrm{glm}$. Para o método de pesos FC, embora fosse possível utilizar as funções semicure e gfcure, respectivamente, para o modelo semiparamétrico e Weibull, foram utilizadas as mesmas rotinas criadas para os modelos ponderados descritas a seguir, que ajustam modelos com fração de cura usuais quando os pesos não são especificados.

Para acomodar os pesos no ajuste dos modelos finais semiparamétricos, a rotina semicure foi modificada. Foi criada a função semicurewei que inclui a opção weights. Nesta opção, deve-se especificar a variável do banco de dados que contém os pesos para cada indivíduo. Para calcular a função de sobrevivência padrão incluindo a ponderação, foi necessário criar uma nova função dentro da rotina semicurewei. Isto porque a função 
survfit que faz parte da library survival do R e é chamada pelo programa semicure, não aceita como objeto ajustes de modelos de Cox ponderados.

A maior dificuldade na obtenção dos resultados foi em relação ao tempo de processamento. Conforme descrito anteriormente, para ajustar o modelo com fração de cura semiparamétrico, é preciso obter as estimativas da função de sobrevivência para cada indivíduo, que por sua vez dependem da função de sobrevivência padrão calculada em cada tempo distinto de falha. Portanto, quanto maior o número de indivíduos e de falhas, maior o tempo de processamento.

No banco de dados utilizado neste trabalho havia 898 indivíduos e 263 tempos distintos de falha. O programa semicure levou 58 minutos para ajustar o modelo com estes dados. Este tempo foi consideravelmente reduzido quando foi executado o programa semicurewei. Isto pode ser explicado pelo fato que a função survfit utilizada pelo semicure calcula a curva de sobrevivência para cada indivíduo, ou seja, calcula a probabilidade de sobrevivência estimada para cada indivíduo em cada tempo distinto de falha. Isto significa que para este banco de dados, o programa calcula 263 estimativas para cada um dos 898 indivíduos. Este cálculo não é necessário para o processo de estimação dos parâmetros de interesse, pois o que é de fato utilizado são apenas as estimativas das probabilidades de sobrevivência nos tempos de falha ou censura observados, o que implica em obter uma única estimativa para cada indivíduo $\left(\hat{S}\left(t_{i}\right)\right)$, além das estimativas padrão calculadas nos tempos de falha. Com isso, o programa modificado calcula um vetor no lugar de uma matriz e o ajuste do modelo semiparamétrico é obtido em aproximadamente 6 minutos.

Para o modelo paramétrico, não foi possível modificar a rotina gfcure, pois a função principal de ajuste, criada na linguagem $\mathrm{C}$ e chamada pela rotina em $\mathrm{R}$, só estava disponível em um arquivo no formato dll (Dynamic-Link Library), cujo código fonte não era público. Neste caso, a implementação dos modelos Weibull foi feita utilizando 
a rotina de maximização $n l m$ do $\mathrm{R}$. A rotina criada foi chamada de weicure.

A modificação do programa para o modelo semiparamétrico trouxe uma redução significativa no tempo de processamento. Por outro lado, o programa que utiliza a função $n l m$ para os modelos Weibull mostrou-se muito mais lento que o gfcure. Para o mesmo banco de dados, o gfcure é executado instantaneamente, enquanto que o weicure leva aproximadamente 9 minutos.

Para os resultados do Capítulo 5, para cada amostra foram realizados os seguintes passos:

- Simulação dos dados omissos. As probabilidades que determinam a omissão foram geradas através da função runif que gera variáveis aleatórias na Uniforme $(0,1)$. Foram criadas duas novas variáveis no banco de dados: um indicador de omissão e a variável fração de ejeção com os dados omissos simulados.

- Ajuste do modelo para os pesos logísticos. Utilizou-se a função glm com distribuição binomial e função de ligação logito, considerando como resposta o indicador de omissão e como covariáveis sexo, idade, Chagas, tempo de falha ou censura e indicador de falha.

- Ajuste do modelo para os pesos FC. No caso do modelo semiparamétrico, utilizouse a função semicurewei e, para o modelo Weibull, utilizou-se a função weicure, ambas sem especificar a opção weights. Nestes modelos, tanto na parte de sobrevivência quanto na parte de cura, as covariáveis consideradas foram sexo, idade, Chagas e indicador de omissão.

- Ajuste do modelo sem ponderação (ACC). As funções utilizadas foram as mesmas no passo anterior, porém utilizando a covariável fração de ejeção no lugar do indicador de omissão. 
- Ajuste dos modelos ponderados. Neste caso, as funções semicurewei e weicure foram utilizadas especificando na opção weights a variável correspondente aos pesos (logísticos ou FC).

Convém lembrar que, em cada cenário (baixo, médio, alto), em cada tipo de modelo (Weibull e Cox), considerou-se 500 amostras com dados omissos simulados. Em cada amostra, foram realizados 5 ajustes: modelo para estimar os pesos logísticos, modelo para estimar os pesos FC e modelos finais considerando os três métodos (sem ponderação, ponderação logística e FC). Para executar os processos, foram utilizadas várias máquinas que trabalharam simultaneamente. Os tempos mencionados como exemplo foram obtidos com um processador Pentium 4, CPU 3.20GHz com 1GB de RAM e sistema operacional Windows XP.

As rotinas criadas para implementar os modelos com fração de cura ponderados serão disponibilizadas em ftp://ftp.ime.usp.br/pub/acarlos/atpaes. 


\section{Referências Bibliográficas}

Andersen, P., Borgan, O., Gill, R. \& Keiding, N. (1993). Statistical Models Based on Counting Processes, Springer-Verlag, New York.

Andersen, P. \& Gill, R. (1982). Cox's regression model for counting processes: A large sample study, The Annals of Statistics 10: 1100-1120.

Baker, S. (1994). Regression analysis of grouped survival data with incomplete covariates: Nonignorable missing-data and censoring mechanisms, Biometrics 50: 821826.

Berkson, J. \& Gage, R. (1952). Survival curve for cancer patients following treatment, Journal of the American Statistical Association 47: 501-515.

Binder, D. (1992). Fitting Cox's proportional hazards models from survey data, Biometrika 79: 139-147.

Blackhurst, D. M. \& Schluchter, M. D. (1989). Logistic regression with a partially observed covariate, Communications in Statistics - Simulation and Computation 18: $163-177$.

Boag, J. (1949). Maximum likelihood estimates of the proportion of patients cured by cancer theraphy, Journal of the Royal Statistical Society, Ser. B 11: 15-44.

Chen, H. (2002). Double-semiparametric method for missing covariates in Cox regression models, Journal of the American Statistical Association 97(458): 565-576. 
Chen, H. \& Little, R. J. A. (1999). Proportional hazards regression with missing covariates, Journal of the American Statistical Association 94(447): 896-908.

Chen, M. \& Ibrahim, J. G. (2001). Maximum likelihood methods for cure rate models with missing covariates, Biometrics 57(1): 43-52.

Chen, M., Ibrahim, J. G. \& Lipsitz, S. R. (2002). Bayesian methods for missing covariates in cure rate models, Lifetime Data Analysis 8: 117-146.

Cho, M. H., Schenker, N., Taylor, J. M. \& Zhuang, D. (2001). Survival analysis with long-term survivors and partially observed covariates, The Canadian Journal of Statistics 29(3): 421-436.

Cox, D. (1972). Regression models and life-tables (with discussion), Journal of the Royal Statistical Society, Ser. B 34: 187-220.

Cox, D. (1975). Partial likelihood, Biometrika 62: 269-276.

Davison, A. C. \& Hinkley, D. V. (1997). Bootstrap methods and their application, Cambridge University Press, New York.

Demissie, S., LaValley, M. P., Horton, N. J., Glynn, R. J. \& Cupples, L. (2003). Bias due to missing exposure data using complete-case analysis in the proportional hazards regression model, Statistics in Medicine 22: 545-557.

Diggle, P. \& Kenward, M. G. (1994). Informative drop-out in longitudinal data analysis, Applied Statistics 43(1): 49-93.

Efron, B. (1982). The Jackknife, the Bootstrap and other resampling plans, SIAM, Philadelphia. 
Fahrmeir, L. \& Kaufmann, H. (1985). Consistency and asymptotic normality of the maximum likelihood estimator in generalized linear models, The Annals of Statistics 13: $342-68$.

Fang, H., Li, G. \& Sun, J. (2005). Maximum likelihood estimation in a semiparametric logistic/proportional hazards mixture model, Scandinavian Journal of Statistics 32: $59-75$.

Farewell, V. (1982). The use of mixture models for the analysis of survival data with long-term survivors, Biometrics 38: 1041-1046.

Folmann, D. \& Wu, M. (1995). An approximate generalized linear model with random effects for informative missing data, Biometrics 51: 151-168.

Freitas, H., Chizzola, P., Paes, A., Lima, A. \& Mansur, A. J. (2005). Risk stratification in a brazilian hospital-based cohort of 1220 outpatients with heart failure: role of Chagas'heart disease., International Journal of Cardiology 102(2): 239-247.

Gagnon, D., Glickman, M., Myers, R. \& Cupples, L. (2003). The analysis of survival data with a non-susceptible fraction and dual censoring mechanisms, Statistics in Medicine 22: 3249-3262.

Ghitany, M. \& Maller, R. (1992). Asymptotic results for exponential mixture models with long-term survivors, Statistics 23: 321-336.

Ghitany, M., Maller, R. \& Zhou, S. (1994). Exponential mixture models with long-term survivors and covariates, Journal of Multivariate Analysis 49: 218-241.

Gibbons, L. \& Hosmer, D. (1991). Conditional logistic regression with missing data, Communications in Statistics - Simulation and Computation 20: 109-120. 
Herring, A. H. \& Ibrahim, J. (2001). Likelihood-based methods for missing covariates in the Cox proportional hazards model, Journal of the American Statistical Association 96(453): 292-302.

Herring, A. H., Ibrahim, J. \& Lipsitz, S. R. (2002). Frailty models with missing covariates, Biometrics 58: 98-109.

Herring, A. H., Ibrahim, J. \& Lipsitz, S. R. (2004). Non-ignorable missing covariate data in survival analysis: a case-study of an international breast cancer study group trial, Journal of the Royal Statistical Society, Ser. C 53: 293-310.

Horton, N. J. \& Laird, N. M. (1998). Maximum likelihood analysis of generalized linear models with missing covariates, Statistical Methods in Medical Research 8: 37-50.

Ibrahim, J. G. (1990). Incomplete data in generalized linear models, Journal of the American Statistical Association 85(411): 765-769.

Ibrahim, J. G., Chen, M. H. \& Lipsitz, S. R. (1999). Monte Carlo EM for missing covariates in parametric regression models, Biometrics 55: 591-596.

Ibrahim, J. G., Chen, M. H. \& Lipsitz, S. R. (2002). Bayesian methods for generalized linear models with missing covariates, The Canadian Journal of Statistics 30: 5578.

Ibrahim, J. G., Chen, M. H., Lipsitz, S. R. \& Herring, A. (2005). Missing-data methods for generalized linear models: a comparative review, Journal of the American Statistical Association 100(469): 332-346.

Kalbfleisch, J. \& Prentice, R. (2002). The Statistical Analysis of Failure Time Data, 2 edn, Wiley, New York.

Kuk, A. Y. C. \& Chen, C. (1992). A mixture model combining logistic regression with proportional hazards regression, Biometrika 79: 531-41. 
Lam, K, F. \& Xue, H. (2005). A semiparametric regression cure model with current status data, Biometrika 92(3): 573-586.

Leong, T., Lipsitz, S. R. \& Ibrahim, J. G. (2001). Incomplete covariates in the Cox model with applications to biological marker data, Applied Statistics 50: 467-484.

Li, Y. \& Feng, J. (2005). A nonparametric comparison of conditional distributions with nonnegligible cure fractions, Lifetime Data Analysis 11: 367-387.

Lin, D. Y. \& Ying, Z. (1993). Cox regression with incomplete covariate measurements, Journal of the American Statistical Association 88(424): 1341-1349.

Lipsitz, S. R. \& Ibrahim, J. G. (1996a). A conditional model for incomplete covariates in parametric regression models, Biometrika 83: 916-922.

Lipsitz, S. R. \& Ibrahim, J. G. (1996b). Using the EM-algorithm for survival data with incomplete categorical covariates, Lifetime Data Analysis 2: 5-14.

Lipsitz, S. R. \& Ibrahim, J. G. (1998). Estimating equations with incomplete categorical covariates in the Cox model, Biometrics 54: 1002-1013.

Lipsitz, S. R., Ibrahim, J. G. \& Zhao, L. P. (1999). A weighted estimating equation for missing covariate data with properties similar to maximum likelihood, Journal of the American Statistical Association 94(448): 1147-1160.

Lipsitz, S. R., Parzen, M. \& Ewell, M. (1998). Inference using conditional logistic regression with missing covariates, Biometrics 54: 295-303.

Little, R. \& Rubin, D. (2002). Statistical Analysis with Missing Data, WileyInterscience, New Jersey.

Little, R. \& Schluchter, M. (1985). Maximum likelihood estimation for mixed continuous and categorical data with missing values, Biometrika 72: 497-512. 
Lu, W. \& Ying, Z. (2004). On semiparametric transformation cure models, Biometrika 91(2): 331-343.

Maller, R. \& Zhou, X. (1996). Survival Analysis with Long-Term Survivors, Wiley Series, Chichester.

Martinussen, T. (1999). Cox regression with incomplete covariate measurements using the em-algorithm, Scandinavian Journal of Statistics 26: 479-491.

McCullagh, P. \& Nelder, J. (1989). Generalized Linear Models, Chapman and Hall, London.

Ng, S. K., McLachlan, G., Yau, K. K. \& Lee, A. (2004). Modelling the distribution of ischaemic stroke-specific survival time using an EM-based mixture approach with random effects adjustment, Statistics in Medicine 23: 2729-2744.

Paik, M. C. \& Tsai, W. Y. (1997). On using the Cox proportional hazards model with missing covariates, Biometrika 84: 579-593.

Peng, Y. (2003a). Estimating baseline distribution in proportional hazards cure models, Computational Statistics \& Data Analysis 42: 187-201.

Peng, Y. (2003b). Fitting semiparametric cure models, Computational Statistics and Data Analysis 41: 481-490.

Peng, Y. \& Dear, K. (2000). A nonparametric mixture model for cure rate estimation, Biometrics 56: 237-243.

Peng, Y., Dear, K. B. G. \& Denham, J. W. (1998). A generalized F mixture model for cure rate estimation, Statistics in Medicine 17: 813-830.

Pierce, D. A., Stewart, W. H. \& Kopecky, K. (1979). Distribution-free regression analysis of grouped survival data, Biometrics 35(4): 785-793. 
Pugh, M., Robbins, S., Lipsitz, S. \& Harrington, D. (1993). Inference in the Cox proportional hazards model with missing covariates, Technical Report 758Z, Department of Biostatistics, Harvard School of Public Health, Boston. .

Rebolledo, R. (1980). Central limit theorems for local martingales, Z. Wahsch verw. Geb. 51: 269-286.

Robins, J. M., Rotnitzky, A. \& Zhao, L. P. (1994). Estimation of regression coefficients when some regressors are not always observed, Journal of the American Statistical Association 89(427): 846-866.

Schemper, M. \& Smith, T. (1990). Efficient evaluation of treatment effects in the presence of missing covariate values, Statistics in Medicine 9: 777-784.

Schluchter, M. D. \& Jackson, K. L. (1989). Log-linear analysis of censored survival data with partially observed covariates, Journal of the American Statistical Association 84(405): 42-52.

Self, S. \& Prentice, R. (1986). Asymptotic distribution theory and efficiency results for case-cohort studies, The Annals of Statistics 16: 64-81.

Sen, P. \& Singer, J. (1993). Large Sample Methods in Statistics, Chapman \& Hall, New York.

Sy, J. \& Taylor, J. (2000). Estimation in a Cox proportional hazards cure model, Biometrics 56: 227-236.

Taylor, J. (1995). Semi-parametric estimation in failure time mixture models, Biometrics 51: 899-907.

Therneau, T. \& Grambsch, P. (2000). Modeling Survival Data, Springer-Verlag, New York. 
Vach, W. (1994). Logistic regression with missing values in the covariates, SpringerVerlag, New York.

Wu, M. C. \& Follmann, D. (1999). Use of summary measures to adjust for informative missingness in repeated measures data with random effects, Biometrics 55: 75-84.

Yin, G. \& Ibrahim, J. G. (2005a). Cure rate models: a unified approach, The Canadian Journal of Statistics 33(4): 559-570.

Yin, G. \& Ibrahim, J. G. (2005b). A general class of bayesian survival models with zero and nonzero cure fractions, Biometrics 61: 403-412.

Zhao, X., Lipsitz, S. R. \& Lew, D. (1996). Regression analysis with missing covariate data using estimating equations, Biometrics 52: 1165-1182.

Zhao, X. \& Zhou, X. (2006). Proportional hazards models for survival data with long-term survivors, Statistics $\&$ Probability Letters 76: 1685-1693.

Zhou, H. \& Pepe, M. S. (1995). Auxiliary covariate data in failure time regression, Biometrika 82: 139-149. 